\title{
Assessing Composite and Fibre Metal Laminate Materials for Automotive Applications Through Impact and Quasi-static Indentation Testing
}

\author{
by
}

\section{Robert Desnoo}

A thesis submitted to the Faculty of Graduate Studies and Research in partial fulfillment of the requirements for the degree of Master of Applied Science

in

Mechanical Engineering

Ottawa-Carleton Institute of Mechanical and Aerospace

Engineering

Carleton University

Ottawa, Ontario

September 2015

Copyright $(C$

2015, Robert Desnoo 


\begin{abstract}
Automakers can conform to pressures of increasing gas mileage and reducing $\mathrm{CO}_{2}$ emissions through weight reduction of vehicles with the use of composite and fibre metal laminate (FML) materials. An important safety equivalency characteristic of these lightweight materials that must be studied is their impact resistance compared to that of traditionally used automotive materials; steel and aluminum. Low-velocity impact (LVI) and quasi-static indentation (QSI) testing was conducted on thin composite and FML panels to assess their applicability in impact prone automotive components. To aide in the impact assessment of the lightweight materials a novel approach was developed to determine the strain and visible damage evolution within specimens through the use of digital imaging correlation (DIC) technology in quasi-static indentation tests. Simulating dynamic impact events with quasi-static loads was also evaluated and its limitations were discussed.
\end{abstract}

The impact characteristics of monolithic aluminum 2024-T3 sheet outperformed carbon/epoxy, carbon/nylon, CARAL 5 2/1-0.3, and GLARE 5 2/1-0.3 lightweight panels. Due to the strain rate strengthening effects of glass fibres, GLARE was proven to be the best alternative to aluminum for automotive applications. The use of quasi-static loading to replicate dynamic impacts was validated for CARAL, aluminum 2024-T3, and GLARE. DIC technology was successfully implemented in QSI tests where full field deformation data provided unmatched detail of visible damage and crack initiation progression. 


\section{Acknowledgements}

The completion of this thesis would not have been possible without the support and encouragement of those around me. First and foremost, I would like to thank my supervisors Dr. Xiao Huang and Dr. Calvin Rans for their continued support throughout my thesis and for providing me with the opportunity to undertake a portion of my thesis at TU Delft. I also owe acknowledgement to the technicians at the CU MAAE Machine Shop and TU Delft's DASML (Alex Proctor, Kevin Sangster, Ian Loy, Cees Paalvast, Bob de Vogel and Berthil Grashof), for their guidance and help in completing my testing. Last but not least, I would like to thank my wife, Suzan Bsat, for always believing in me and reassuring me when I needed it the most. 


\section{Table of Contents}

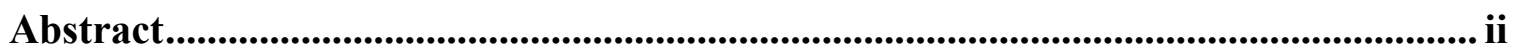

Acknowledgements ............................................................................................................................ iii

Table of Contents ...................................................................................................................... iv

List of Tables .................................................................................................................... vii

List of Figures.................................................................................................................. viii

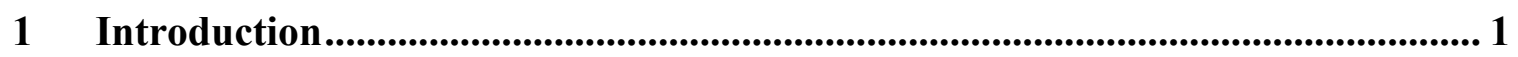

2 Background ................................................................................................................ 4

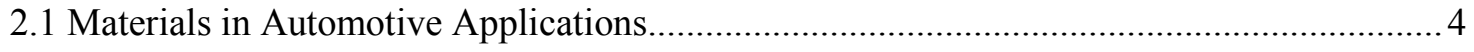

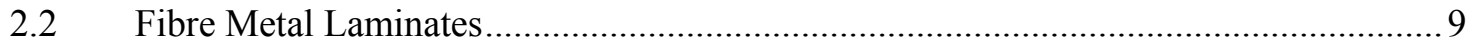

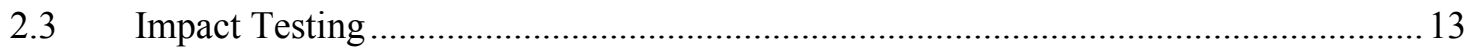

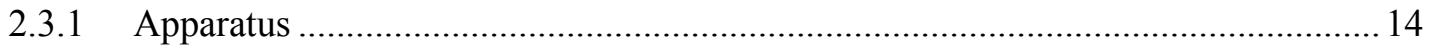

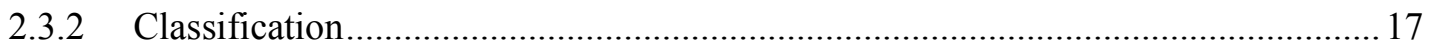

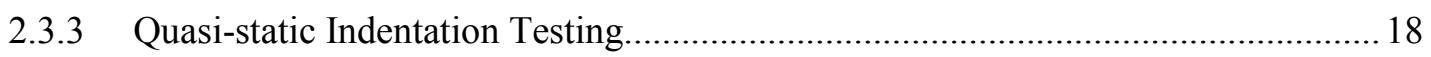

2.3.4 Impact Testing for Automotive Applications......................................................... 19

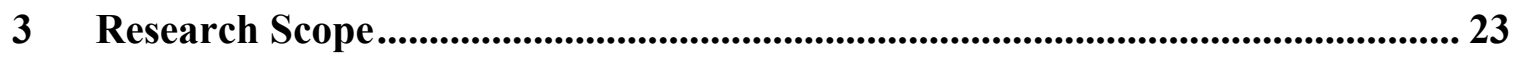

4 Materials and Experimental Procedures ................................................................. 25

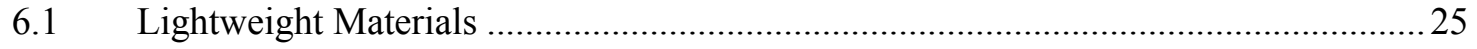

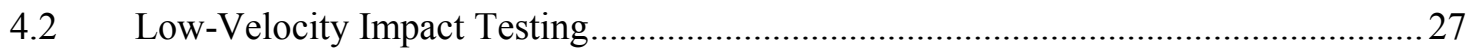

4.3 Aluminum 2024-T3 Low-Velocity Impact Finite Element Model..................................30

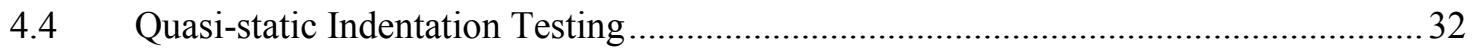

5 Methodology for Approaching and Verifying Stereovision DIC Setups ........... 35

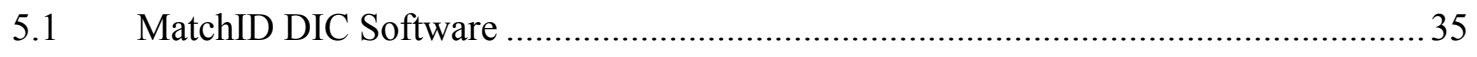




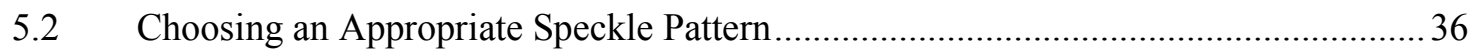

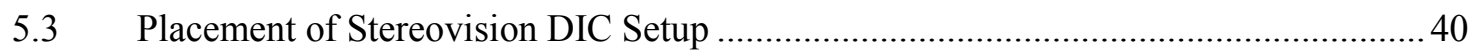

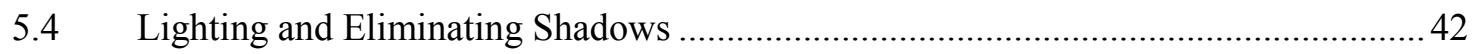

5.5 Validating DIC Data through Displacement Rates.................................................. 44

5.6 Validating DIC Data with a Secondary Strain Measuring Technique.......................... 46

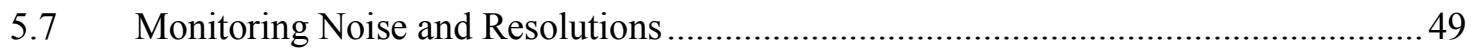

5.8 DIC Testing Procedure and Summary of Parameters.................................................52

6 Results and Discussion............................................................................................. 55

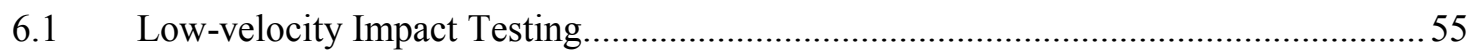

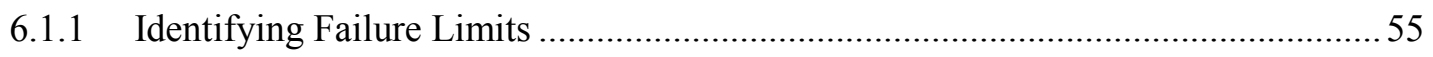

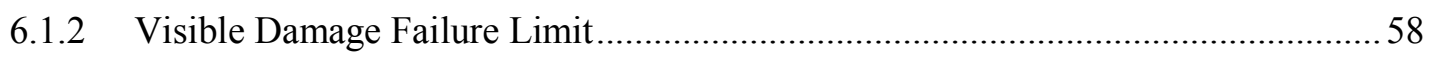

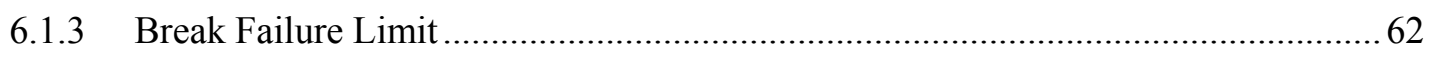

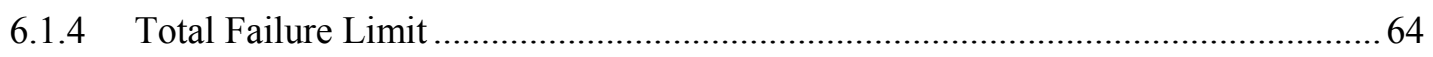

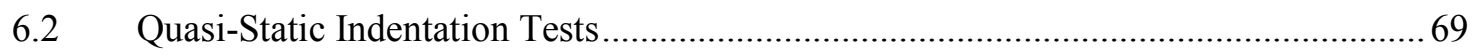

6.2.1 Comparing Quasi-Static Indentation to Low-Velocity Impacts .............................. 69

6.2.2 Advantages of Using DIC in Quasi-static Indentation Tests ................................ 82

6.2.2.1 Out-of-Plane Displacement Profiles......................................................... 82

6.2.2.2 Quantifying Visible Yield Damage …........................................................ 85

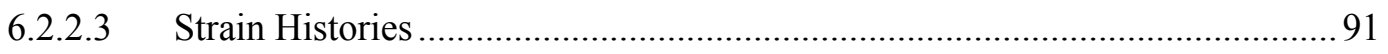

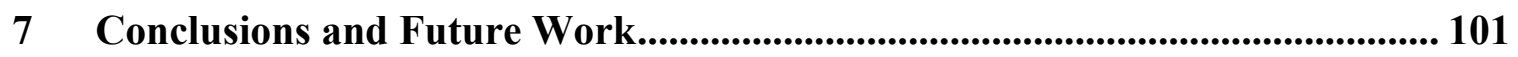

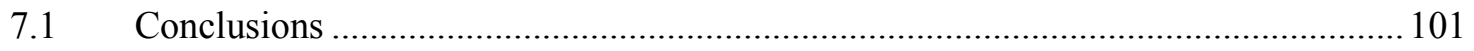

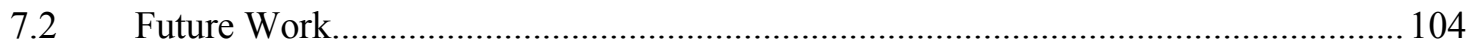

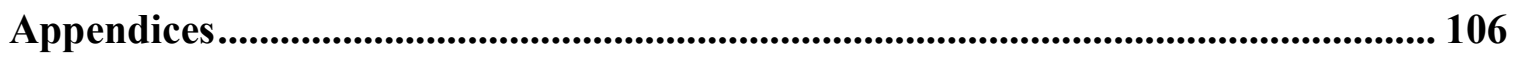

Appendix A Non-Destructive Inspection of Manufactured Panels ....................................... 106

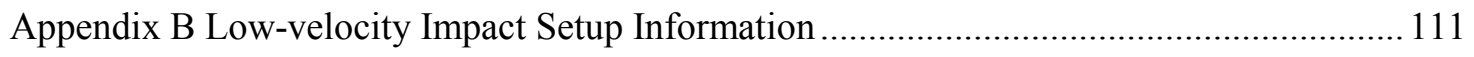


Appendix C DIC Terminology and Parameters..

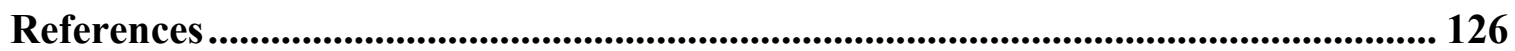




\section{List of Tables}

Table 1: Strength, cost and possible mass reduction characteristics of potential

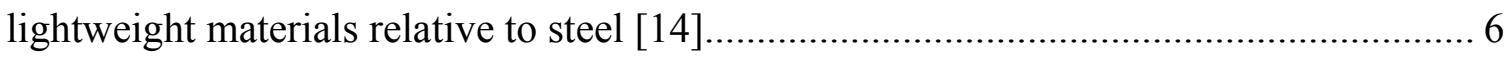

Table 2: Mechanical properties of typical automotive aluminum alloys [16-18].............. 7

Table 3: Layup characteristics and structural benefits for standard grades of GLARE [27]

Table 4: Performance criterion and force-deflection features of impact failure limits [66]

Table 5: Mechanical properties of laminate constituents ............................................... 25

Table 6: Configuration of materials tested............................................................. 27

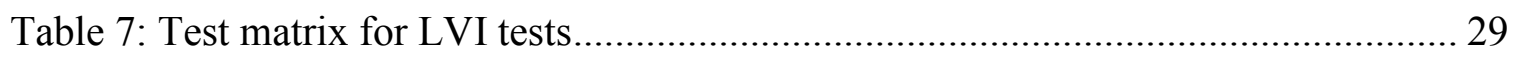

Table 8: Aluminum 2024-T3 material properties ....................................................... 31

Table 9: Set deflection values based on $80 \%$ of deflection at maximum contact force ... 33

Table B1: Impactor mass and velocity at each impact energy tested ............................. 111

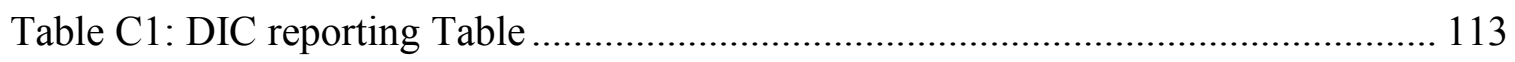

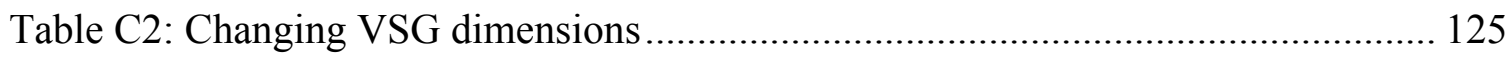




\section{List of Figures}

Figure 1: Breakdown of materials in the 2015 Ford F-150 [8] ................................... 2

Figure 2: The progression of lightweight materials in a typical United States family

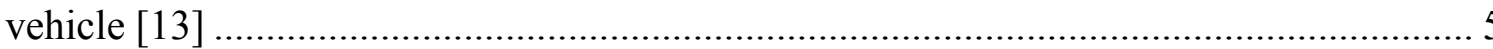

Figure 3: Cost related to different designs, weight and rate of lightweight construction

[22]

8

Figure 4: Lay-up of a standard cross-ply GLARE FML [28] .................................... 9

Figure 5: Effect of strain rate on the stress-strain curve of aluminum 2024-T3 (reduced) $[26,33]$ and GLARE 3 (7075) [32]

Figure 6: Typical load and energy versus time curve and characteristic points for post impact analysis [44]

Figure 7: Typical force-deflection responses from LVI tests of a.) a ductile material and b.) a fibre reinforced material [66]

Figure 8: Frequency of occurrence for response features in the characterization of six example applications [66] 22

Figure 9: Clamping fixture and impactor for LVI and QSI tests ................................. 28

Figure 10: Aluminum 2024-T3 low-velocity impact finite element model..................... 31 Figure 11: Force-deflection curves of aluminum 2024-T3 from low-velocity impact FEA simulation and experimental LVI tests 32

Figure 12: Critical DIC parameters in the MatchID software and how they relate to the captured image [73] 
Figure 13: Sample containing three different speckle patterns a.) before indentation b.) minimum principal strain E2 fringe plot captured during indentation 38

Figure 14: Exx plotted on a.) $0.1 \mathrm{~mm}$ pen speckle pattern for a subset size of 21 and b.) on $0.8 \mathrm{~mm}$ pen speckle pattern for a subset size of 41 39

Figure 15: a.) Exx plotted on a spray painted low density speckle pattern for a subset size of 11 b.) E2 plotted on a high density speckle pattern for a subset size of $11 \ldots \ldots \ldots \ldots \ldots . . . .40$

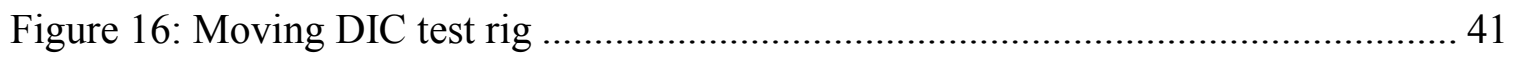

Figure 17: a.) change in displacement and strain resolutions and b.) change in noise for a

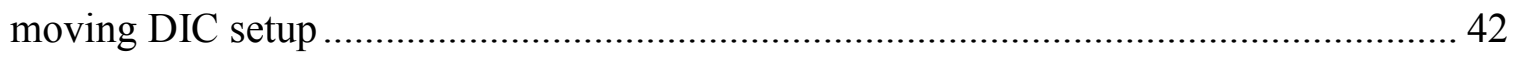

Figure 18: 3D projection of undeformed GLARE specimen..................................... 43

Figure 19: Shadowing on different test fixture backgrounds a.) bare metal b.) spray

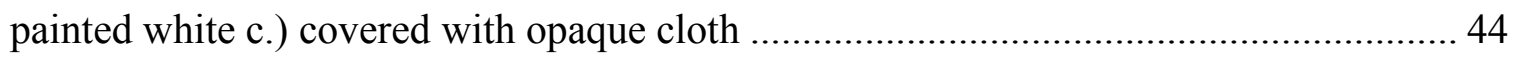

Figure 20: 9x12 DIC calibration panel with $3 \mathrm{~mm}$ grid spacing ............................... 45

Figure 21: Comparing DIC displacement rates after calibration with different panel sizes

Figure 22: Out-of-plane (Z-direction) position fringe plot showing the fibre optic strain

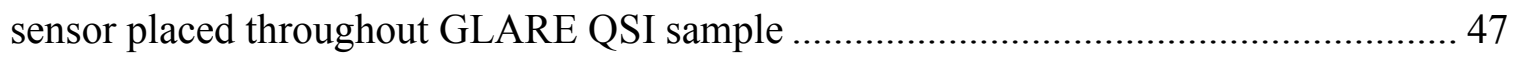
Figure 23: Comparing DIC and fibre optic strain data at an indentation time of 20 (s) for

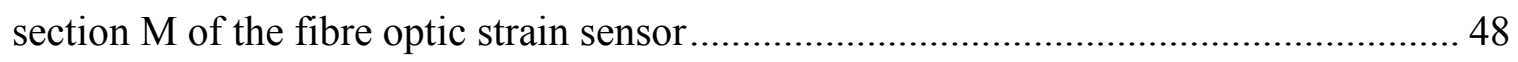
Figure 24: Comparing DIC and fibre optic strain data at an indentation time of 260 (s) for

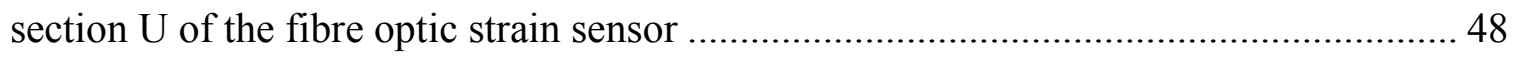

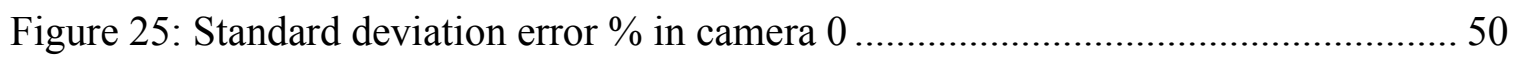

Figure 26: Comparison of camera noise with changing test fixture surface................... 51 
Figure 27: DIC resolutions a.) out-of-plane displacement b.) in-plane strain ................. 51

Figure 28: Force-deflection plots from LVI tests highlighting failure limits .................. 56

Figure 29: Visible damage states on the non-impact side that occur before the proportional limit is reached for a.) carbon/nylon and b.) carbon/epoxy ....................... 57

Figure 30: Plastic deformation at impact site and in unsupported region of a.) aluminum

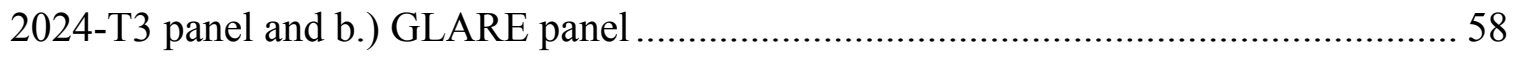

Figure 31: Maximum and permanent deflections at increasing low energy impacts ....... 59

Figure 32: Impact parameters at the first sign of visible damage on the non-impact side 61

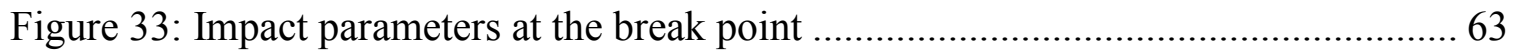

Figure 34: Energy profile diagram emphasizing impact energies to cause puncture ....... 65

Figure 35: Stress-strain curves of the various laminates and their constituents ............... 66

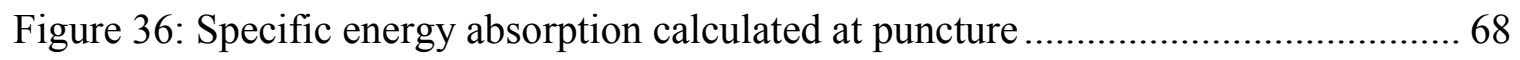

Figure 37: LVI test contact forces (solid line) and central deflections (dotted line) as a

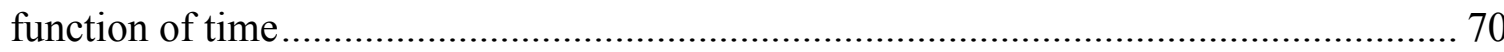

Figure 38: Contrasting a.) maximum contact force b.) Central deflection at maximum

contact force and c.) energy absorbed between LVI and QSI tests at puncture .............. 72

Figure 39: Damage states at puncture in carbon/epoxy for a.) QSI test showing significant plate bending and membrane deformation and b.) LVI test showing brittle fracture....... 73

Figure 40: Damage states at puncture in carbon/nylon for a.) QSI test showing significant plate bending and membrane deformation and b.) LVI test showing brittle fracture....... 74 Figure 41: Damage states at puncture on the impact side in CARAL for a.) QSI test b.)

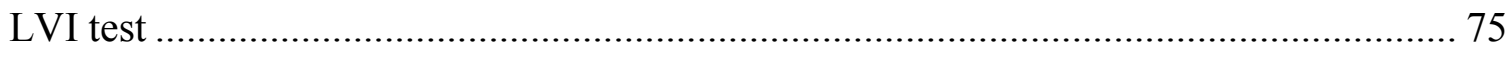


Figure 42: Dependency of strain rate on impactor velocity in FEA low-velocity impact

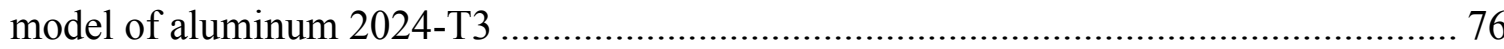

Figure 43: Comparing force-deflection curves for QSI and LVI tests on GLARE .......... 77 Figure 44: LVI and QSI parameter comparability for increasing LVI impactor velocity in a.) GLARE and b.) aluminum 2024-T3 79 Figure 45: Chemically etched samples of a.) GLARE QSI specimen and b.) GLARE LVI specimen at puncture. 80

Figure 46: Chemically etched samples of CARAL at an impact/indent energy of $12.55 \mathrm{~J}$ for a.) QSI and b.) LVI and GLARE at an impact/indent energy of $25 \mathrm{~J}$ for c.) QSI and d.) LVI. 81

Figure 47: a.) out-of-plane displacement fringe plot for aluminum 2024-T3 at an indentation time of 320 (s) showing data extraction line b.) out-of-plane displacement profiles of GLARE and aluminum 2024-T3 at increasing indentation times . 84 Figure 48: 3D Cartesian strain plots of GLARE depicting plastic deformation at a.) 40 (s) indentation time and b.) 100 (s) indentation time 86 Figure 49: Maximum principal strain fringe plots at 70 (s) for a.) aluminum 2024-T3 and b.) GLARE 87 Figure 50: Progression of plastic deformation area in GLARE and aluminum 2024-T3. 88 Figure 51: QSI force-deflection curves of aluminum 2024-T3 and GLARE taken to puncture. 89

Figure 52: LVI force-deflection curves of aluminum 2024-T3 and GLARE at low impactor velocities 90 
Figure 53: LVI force-deflection curves of aluminum 2024-T3 and GLARE at high

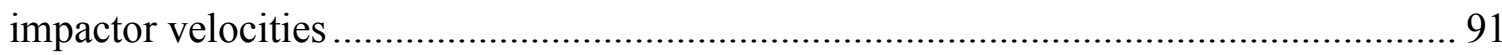

Figure 54: Maximum principal strain profiles at an indentation time of 40 (s).............. 92

Figure 55: Maximum principal strain profiles just before crack initiation on the non-

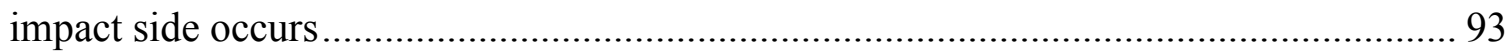

Figure 56: Max principal strain profiles at large central deflections for GLARE and aluminum 2024-T3 exposing differences in friction effects

Figure 57: Correlating QSI E1 strain necking regions to crack locations on the non-

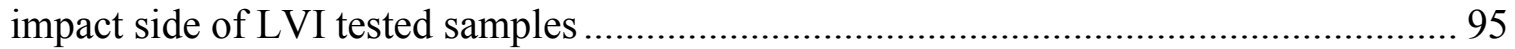

Figure 58: Strain paths in GLARE at the crack initiation site and at the centre of the panel 96

Figure 59: Progression of glass fibre and aluminum 2024-T3 failure in QSI tested GLARE 97

Figure 60: FEA analysis of aluminum 2024-T3 at an impact energy of $125 \mathrm{~J}$ without

friction 99

Figure 61: Comparison of max. principal strains at different $(\mathrm{x}, \mathrm{y})$ positions between DIC indentation tests and a 125 joule FEA impact model 100

Figure A1: C-scan image of (far left) GLARE reference panel, (middle) GLARE panel, and (far right CARAL panel) 106

Figure A2: a.) Top side of GLARE panel with marked defect areas b.) underside of GLARE panel with marked defect areas (30 $\mathrm{mm}$ ruler shown for scale) 107 Figure A3: a.) Top side of CARAL panel with marked defects b.) underside of CARAL panel with marked defects $(30 \mathrm{~mm}$ ruler shown for scale)..... 108 
Figure A4: C-scanned image of (to the left) carbon/epoxy reference panel and (to the right) CARAL panel 109

Figure A5: Carbon/epoxy panel with defect areas outlined (30 $\mathrm{mm}$ ruler shown for scale) 109

Figure A6: C-scan image of carbon/nylon panel (on the left) 110

Figure C1: Impact of noise on DIC results 115

Figure C2: Summary and representation of DIC processing parameters [73]..... 117

Figure C3: A demonstration of how subset size and shape function affect the resolution

Figure C4: The ability of an increasing subset size and varying shape function to capture displacement gradients 119

Figure C5: Displacement field using a subset size of 51

Figure C6: Displacement field using a subset size of 11 120 Figure C7: Horizontal displacement pattern retrieved using a subset size of 51 and affine shape function 121

Figure C8: Horizontal displacement pattern retrieved using a subset size of 11 and affine shape function 121

Figure C9: Horizontal displacement pattern retrieved using a subset size of 51 and quadratic shape function 122

Figure C10: Horizontal displacement pattern retrieved using a subset size of 11 and quadratic shape function 122

Figure C11: DIC processing parameters effect on strain resolution 123 
Figure C12: DIC processing parameters effect on high frequency strain amplitude

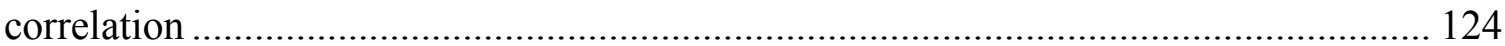




\section{Introduction}

Today's automakers are experiencing increasing pressures to improve gas mileage and reduce the carbon footprint of their vehicles by not only the general public but also world governments [1]. While new hybrid powertrain technologies promise to help reach these goals, the lightweighting of vehicle platforms will improve not only current but future vehicle fuel economy. A vehicle mass reduction of $15 \%$ has shown to decrease fuel consumption by $10 \%$ along with comparable reductions in $\mathrm{CO}_{2}$ emissions [2]. Research has determined that mass reductions greater than $40 \%$ are possible through the use of composite materials, potentially leading to a total vehicle mass savings of $20 \%$ [3].

Automakers in the past have been reluctant to make major changes in manufacturing materials due to the high costs associated with updating factories, production tooling, and engineering expertise [4]. Thus the implementation of lightweight metallic materials other than steel, let alone composite materials, has been non-existent for large production vehicles. However, due to the demands for increasingly hard to achieve fuel economies and emissions, the current trend in the automotive industry has been to replace steel components with aluminum. Use of aluminum alloys in automotive applications has steadily grown and was previously reserved for luxury makes such as Audi where all aluminum bodies were implemented [4]. Now automakers such as Ford have begun to utilize aluminum in high volume production vehicles with other automakers soon to follow [5]. One example of utilizing aluminum to reduce weight is seen in the 2015 Ford 
F-150 pick-up truck where the majority of the body is stamped out of 5000 and 6000 series aluminum alloys (shown in Figure 1) reducing the mass of the vehicle by $450 \mathrm{lbs}$ $[6,7]$.

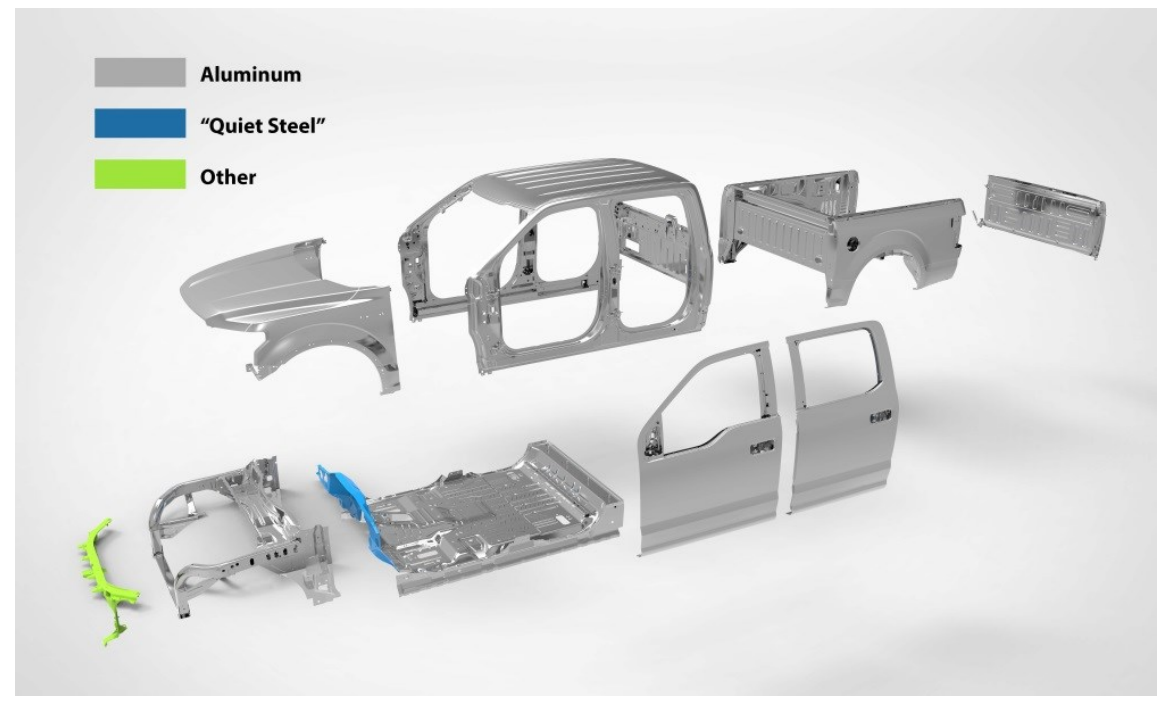

Figure 1: Breakdown of materials in the 2015 Ford F-150 [8]

A major step in the design of the new F-150 pick-up truck was ensuring the aluminum provided the same or higher strength as its steel counterpart. This was achieved through heat treating and increasing the gauge of the aluminum sometimes by as much as 1.7 times the original steel thickness $[6,9]$. Thus engineers made sure the safety and performance of the truck was not compromised by the use of a lighter aluminum material. Engineers at Ford even went so far as to perform custom designed impact tests on the aluminum bed of the truck with train pintle hitches [6]. It is clear that in the future the automotive industry will place a major emphasis on choosing and optimizing lightweight materials for manufacturing. A significant aspect of this material selection process will be the development of new performance tests to assess the performance and ultimately the 
potential of a lightweight material.

This thesis focuses on the assessment of composite and fibre metal laminate lightweight materials for impact prone automotive applications through the use of two main testing methods: 1) instrumented drop weight low-velocity impact and 2) quasi-static indentation tests. The underlying research objective in this work is determining to what extent quasistatic loads can replicate low-velocity impact responses. A novel method for characterising quasi-static indentation events is also introduced where digital image correlation technology will be implemented in QSI tests to provide full field deformation information. 


\section{Background}

\subsection{Materials in Automotive Applications}

Before a material can be used in the automotive industry it must meet requirements that relate to environmental regulations, safety, and consumer demands. These criteria can include: lightweight, cost, safety/crashworthiness, and recycling/ life cycle considerations [10]. Steel has been the material of choice in automotive structures over the past seven decades mostly due to its low cost and good formability [11]. However, with increasing pressure placed on the automotive industry to reduce fuel and greenhouse gas emissions a shift towards lighter material options has already begun. Instead of conventional steels automakers are moving towards the use of higher strength steels, lighter alloys (ie. magnesium and aluminum), and polymer composite materials to reduce vehicle mass [12]. This gradual reduction of conventional steels can be observed in Figure 2 where the weight distribution of automotive materials is given in a typical U.S. family vehicle over a span of 16 years. Two important takeaways from Figure 2 are the slow implementation of lightweight materials in the automotive industry and the increasing vehicle mass with each successive year. Automakers are reluctant to make a switch to new lightweight materials due to the added costs associated with design, factory updates, and production tooling [4]. A rising trend in vehicle mass has been observed due to the increasing requirements for vehicle safety and comfort standards such as consumer demands for space, performance, extra features, and minimal noise and vibrations [12]. 
1995 Model:

Total Vehicle Weight: 3694 lbs
2000 Model:

\section{Total Vehicle Weight: 3902 lbs}

2011 Model:

Total Vehicle Weight: 4072 lbs

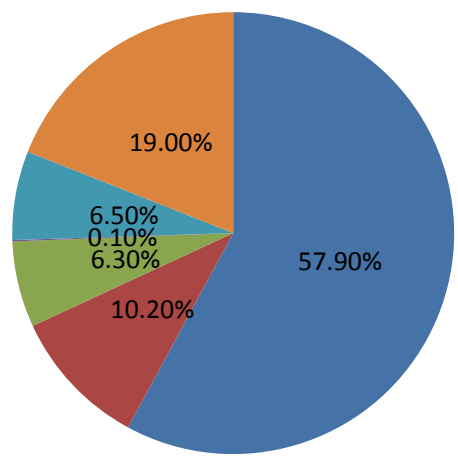

- Iron and Steel

- High and Medium

Strength Steel

Aluminum

Magnesium

Plastics and

Composites

- Other Materials

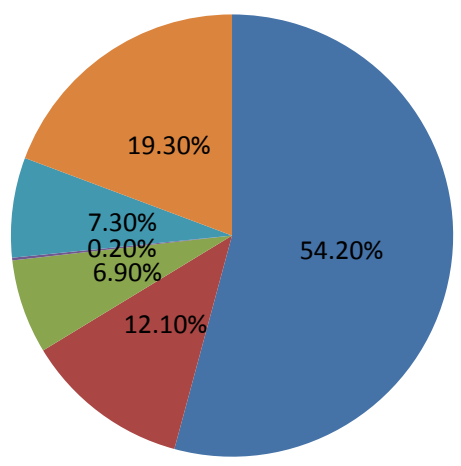

- Iron and Steel

- High and Medium

Strength Steel

Aluminum

nagnesium

- Plastics and

Composites

- Other Materials

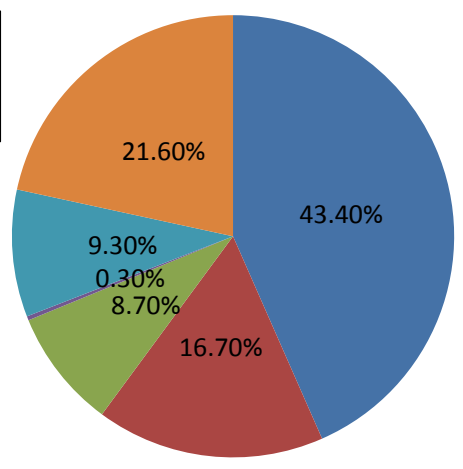

- Iron and Steel

- High and Medium

Strength Steel

- Aluminum

Magnesium

Plastics and

Composites

- Other Materials

Figure 2: The progression of lightweight materials in a typical family vehicle in the United States [13]

To meet stringent fuel consumption and emission regulations the automotive industry will need to achieve vehicle lightweighting through material substitution without affecting the main attributes of the vehicle [12]. A summary of potential lightweight 
materials suitable for automotive structural applications is given in Table 1, along with their strengths, costs and possible mass reductions given relative to conventional steel.

Table 1: Strength, cost and possible mass reduction characteristics of potential lightweight materials relative to steel [14]

\begin{tabular}{|c|c|c|c|c|c|}
\hline Material & $\begin{array}{l}\text { Density } \\
\left(\mathrm{g} / \mathrm{cm}^{3}\right)\end{array}$ & $\begin{array}{l}\text { Strength/ } \\
\text { density }\end{array}$ & $\begin{array}{l}\text { Modulus/ } \\
\text { density }\end{array}$ & Cost & $\begin{array}{c}\text { Mass } \\
\text { reduction } \\
\text { potential (\%) }\end{array}$ \\
\hline & & & \multicolumn{3}{|c|}{ Relative to steel } \\
\hline Mild steel & 7.87 & 1 & 1 & $1 x$ & 0 \\
\hline High strength steel & 7.87 & $1.86 x$ & $1 x$ & $0.9-1.2 x$ & $0-10$ \\
\hline $\begin{array}{l}\text { Advanced high strength } \\
\text { steel }\end{array}$ & 7.87 & $2.25 x$ & $1 x$ & $0.8-1.5 x$ & $0-30$ \\
\hline Aluminum & 2.7 & $3.95 x$ & $1.02 x$ & $1.3-2.0 x$ & $30-60$ \\
\hline Magnesium & 1.74 & $3.66 x$ & $1.02 x$ & $1.5-2.5 x$ & $30-70$ \\
\hline Titanium & 4.51 & $4.73 x$ & $0.98 x$ & $1.5-10.0 x$ & $40-55$ \\
\hline Metal matrix composites & $1.9-2.7$ & $3.81 x$ & $1.45 x$ & $1.5-3.0 x$ & $30-70$ \\
\hline Ceramics & 3.9 & $0.7 x$ & $3.05 x$ & $1.5-3.0 x$ & $10-30$ \\
\hline Plastics & $0.9-1.5$ & $0.82 x$ & $0.08 x$ & $0.7-3.0 x$ & $20-50$ \\
\hline $\begin{array}{l}\text { Sheet moulding } \\
\text { compound }\end{array}$ & 1.1-1.9 & $4.39 x$ & $1.16 \mathrm{x}$ & $0.5-1.5 x$ & $20-30$ \\
\hline Glass fibre composites & $1.4-2$ & $4.74 x$ & $0.5 x$ & $0.9-1.5 x$ & $25-35$ \\
\hline Carbon fibre composites & 1.4-1.6 & $18.3 x$ & $1.93 x$ & $1.5-5.0 x$ & $50-90$ \\
\hline
\end{tabular}

Although carbon fibre composites offer the greatest specific strength and potential for mass reduction their high raw material costs and concerns with recyclability prevent widespread use in the automotive industry $[10,12]$. As a consolation automakers have started to design and manufacture components from less expensive aluminum alloys that have mid-range weight reduction potential when compared to carbon fibre composites. Magnesium alloys offer further weight reduction advantages over aluminum, however, at the expense of mechanical and physical property disadvantages [10]. With significantly lower densities, the difference in strength between aluminum alloys and conventional steels can be overcome by increasing gauge thicknesses to acceptable amounts and age 
hardening heat treatment as was done in the new 2015 Ford F-150 pick-up trucks $[6,9]$.

Lightweight aluminum alloys can reduce the greatest amount of vehicle mass by replacing steel in the main structure known as the Body in White (BIW) (Figure 1) that accounts for $20 \%$ of the vehicles' mass not including closures (doors, hood etc.). Through use of aluminum in the BIW, 20-30\% total vehicle weight savings can be realized [10]. Common aluminum alloys currently employed by the automotive industry are the 5000 and 6000 series alloys $[10,15]$ for which mechanical properties of typical grades are given in Table 2 .

Table 2: Mechanical properties of typical automotive aluminum alloys [16-18]

\begin{tabular}{|c|c|c|c|c|c|c|}
\hline \multirow[t]{2}{*}{ Grade } & \multicolumn{5}{|c|}{ Mechanical Properties } & \multirow{2}{*}{$\begin{array}{l}\text { Automotive } \\
\text { Application }\end{array}$} \\
\hline & $\begin{array}{c}\text { Density } \\
\text { (g/cc) }\end{array}$ & E (GPa) & $\begin{array}{c}\text { YS } \\
(\mathbf{M P a})\end{array}$ & $\begin{array}{c}\text { UTS } \\
\text { (MPa) }\end{array}$ & El\% & \\
\hline 5052-H32 & 2.68 & 70.3 & 193 & 228 & 12 & $\begin{array}{l}\text { Body } \\
\text { Panels }\end{array}$ \\
\hline 6016-T4 & 2.7 & 71 & 110 & 210 & 27 & $\begin{array}{c}\text { Body } \\
\text { Panels }\end{array}$ \\
\hline 6061-T4 & 2.7 & 68.9 & 131 & 228 & 22 & Structural \\
\hline
\end{tabular}

Aluminum alloys appear to be the lightweight material of the near future in the automotive industry or at least until the cost of carbon fibre composites is reduced. Oak Ridge National Laboratory and the United States Automotive Composites Consortium have been working towards this goal as they try to reduce the cost of carbon fibre production to between $\$ 5$ (US) and $\$ 7$ (US) per pound [12]. As an intermediate step, hybrid components composed of fibre reinforced plastics (FRP) and a metallic alloy are increasingly being used in the automotive industry [19]. Combining FRP's and metallic 
alloys allows for exploitation of each material's advantages while overcoming their individual disadvantages. A great example of this would be the combination of carbon fibres' high specific tensile strength with the superior elongation to failure of aluminum. Implementation of hybrid structures has been limited in the automotive industry. Homberg et al. [20] developed a carbon fibre reinforced high strength steel B-pillar at lower cost than pure carbon fibre. Broughton et al. [21] adhered carbon fibre to aluminum extrusions lowering the weight by $33 \%$ over a traditional aluminum optimized structure. Figure 3 provides an overview of the costs and masses linked with material choices in the automotive industry.

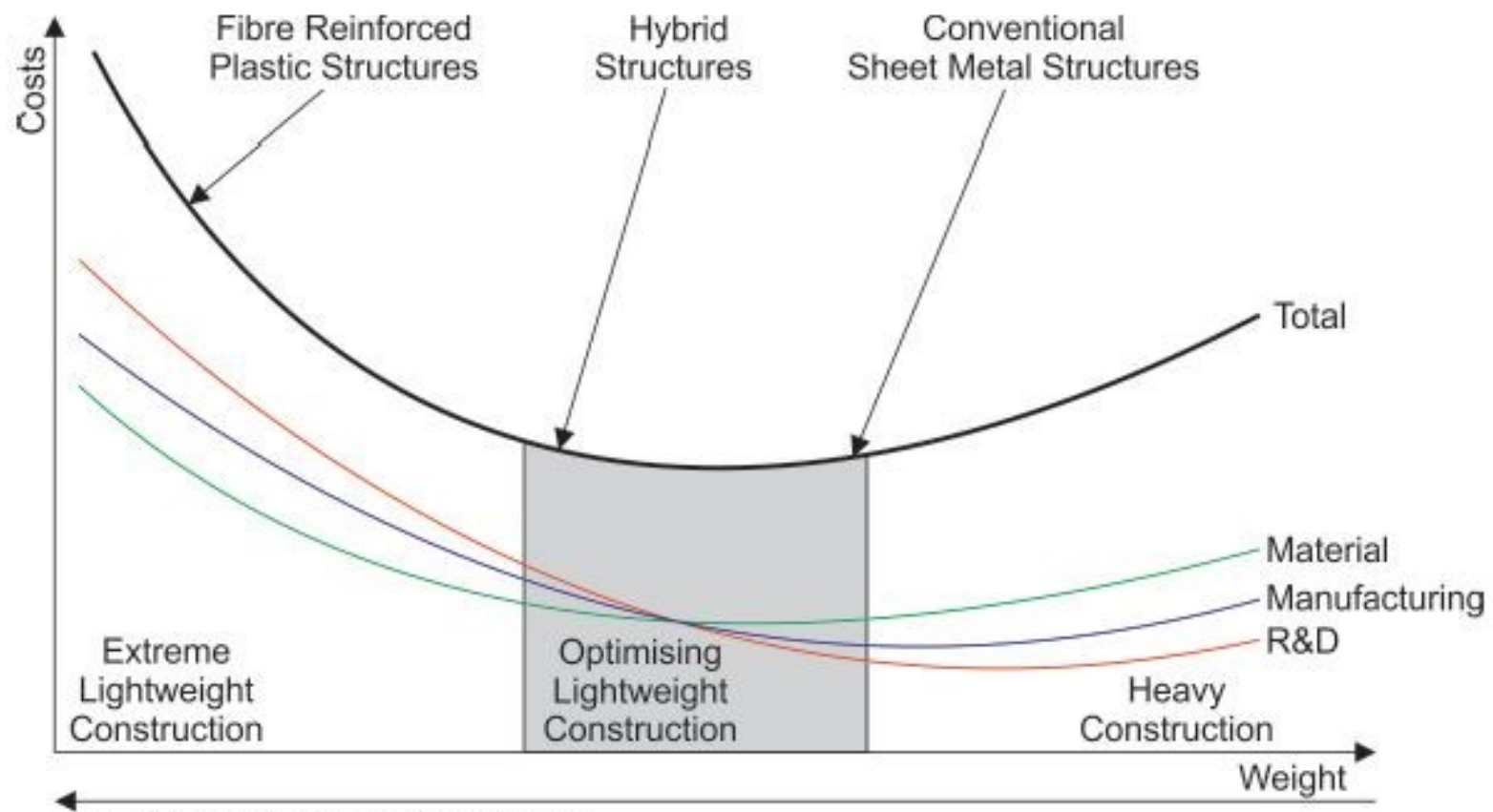

Rate of Lightweight Construction

Figure 3: Cost related to different designs, weight and rate of lightweight construction $[22]$ 
Based on the material, manufacturing, and R\&D costs hybrid structures offer a lighter design option than conventional sheet metal structures at only a slight increase in cost. It is these advantages that have inspired the research conducted in this thesis focusing on the impact resistance of fibre metal laminates for automotive applications.

\subsection{Fibre Metal Laminates}

Fibre metal laminates are defined as a hybrid material consisting of alternating layers of monolithic metallic sheet and pre-impregnated (prepreg) fibre layers (Figure 4) [23]. The development of FMLs dates back to the 1940's [23] and continues today as new pairings of metallic and composite constituents are continuously experimented for engineering applications. Traditional variants of FMLs were developed at the Delft University of Technology (TU Delft) in the 1980's and included aramid, carbon, or E-glass fibre layers with either aluminum 2024-T3 or 7075-T6 metallic layers [24]. At present an FML known as GLARE (GLAss Reinforced aluminum) has become the lightweight material of choice in numerous aerospace applications [25] due to its improved fatigue and impact resistance over monolithic aluminum 2024-T3 and plain composite panels respectively [26, 27]. Standard grades of GLARE consist of unidirectional S2 glass/ FM94 epoxy layers mixed with layers of aluminum 2024-T3 as shown in Figure 4.

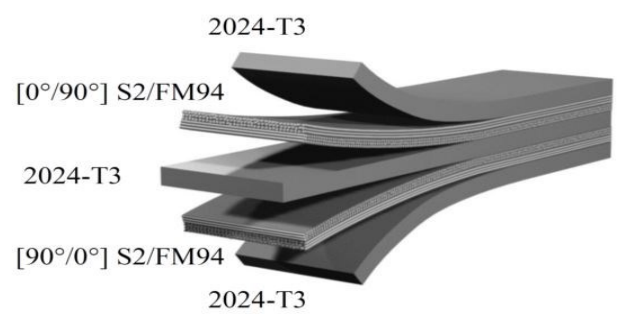

Figure 4: Lay-up of a standard cross-ply GLARE FML [28] 
Six standard grades of GLARE are presented in Table 3 along with their layup characteristics and main structural benefits.

Table 3: Layup characteristics and structural benefits for standard grades of GLARE [27]

\begin{tabular}{|c|c|c|c|c|}
\hline GLARE grade & Sub & $\begin{array}{c}\text { Metal sheet } \\
\text { thickness } \\
\text { [mm] and } \\
\text { alloy }\end{array}$ & $\begin{array}{l}\text { Prepreg } \\
\text { orientation* in } \\
\text { each fibre } \\
\text { laver** }\end{array}$ & $\begin{array}{l}\text { Main beneficial } \\
\text { characteristic }\end{array}$ \\
\hline GLARE 1 & - & $\begin{array}{c}0.3-0.47475- \\
\text { T761 }\end{array}$ & $0 / 0$ & $\begin{array}{c}\text { Fatigue, } \\
\text { strength, yield } \\
\text { stress }\end{array}$ \\
\hline \multirow[t]{2}{*}{ GLARE 2} & GLARE 2A & $\begin{array}{c}0.2-0.52024- \\
\text { T3 }\end{array}$ & $0 / 0$ & $\begin{array}{l}\text { Fatigue, } \\
\text { strength }\end{array}$ \\
\hline & GLARE 2B & $\begin{array}{c}0.2-0.52024- \\
\text { T3 }\end{array}$ & $90 / 90$ & $\begin{array}{l}\text { Fatigue, } \\
\text { strength }\end{array}$ \\
\hline GLARE 3 & - & $\begin{array}{c}0.2-0.52024- \\
\text { T3 }\end{array}$ & $0 / 90$ & $\begin{array}{l}\text { Fatigue, } \\
\text { strength }\end{array}$ \\
\hline \multirow[t]{2}{*}{ GLARE 4} & GLARE 4A & $\begin{array}{c}0.2-0.52024- \\
\text { T3 }\end{array}$ & $0 / 90 / 0$ & $\begin{array}{c}\text { Fatigue, } \\
\text { strength in } 0^{\circ} \\
\text { direction }\end{array}$ \\
\hline & GLARE 4B & $\begin{array}{c}0.2-0.52024- \\
\text { T3 }\end{array}$ & $90 / 0 / 90$ & $\begin{array}{c}\text { Fatigue, } \\
\text { strength in } 90^{\circ} \\
\text { direction }\end{array}$ \\
\hline GLARE 5 & - & $\begin{array}{c}0.2-0.52024- \\
\text { T3 }\end{array}$ & $0 / 90 / 90 / 0$ & Impact \\
\hline \multirow[t]{2}{*}{ GLARE 6} & GLARE 6A & $\begin{array}{c}0.2-0.52024- \\
\mathrm{T} 3\end{array}$ & $+45 /-45$ & $\begin{array}{c}\text { Shear, off-axis } \\
\text { properties }\end{array}$ \\
\hline & GLARE 6B & $\begin{array}{c}0.2-0.52024- \\
\text { T3 }\end{array}$ & $-45 /+45$ & $\begin{array}{l}\text { Shear, off-axis } \\
\text { properties }\end{array}$ \\
\hline
\end{tabular}

*All aluminum rolling directions in standard laminates are in the same orientation; the rolling direction is defined as $0^{\circ}$, the transverse rolling direction is defined as $90^{\circ}$ ** The number of orientations in this column is equal to the number of prepregs (each nominally $0.127 \mathrm{~mm}$ thick) in each fiber layer 
The added advantage of layering FRPs with metallic sheet is the freedom to tailor the hybrid laminate for specific applications as demonstrated in the various GLARE grades. Although primarily considered in design for its excellent fatigue resistance, GLARE and other forms of FMLs are now being optimized for impact prone applications such as aircraft cargo floor panels [26]. Area under a stress-strain plot, known as strain energy density, has been found to be a useful tool for estimating what types of composite and metallic materials may provide leading impact resistance and energy absorption in FMLs [29-31]. Signs of a good impact material are thus large plastic deformation zones (metallic layers) and high failure strains (metallic and composite layers). For these reasons aluminum 2024-T3 is preferred over the stronger and more brittle aluminum 7075-T6 and glass fibres are preferred over the stiffer and more brittle carbon fibres for impact prone structures $[30,31]$. In FMLs such as GLARE it is essential that a good bond exists between the glass and aluminum layers since the panel will be stiffer and able to absorb more impact energy [26]. Another important characteristic to consider is if a material's mechanical properties are strain rate dependent because higher velocity impacts can alter the impact resistance of the material. The strain rate dependency of glass fibres in GLARE is advantageous since the ultimate strength and failure strain of the glass fibres increase at higher impact velocities $[26,32]$. An example of the improved mechanical properties of GLARE 3 (with 7075 aluminum) at a higher strain rate is provided in Figure 5. Also given are the reduced stress-strain profiles of aluminum 2024T3 at quasi-static and high strain rate loading conditions. 


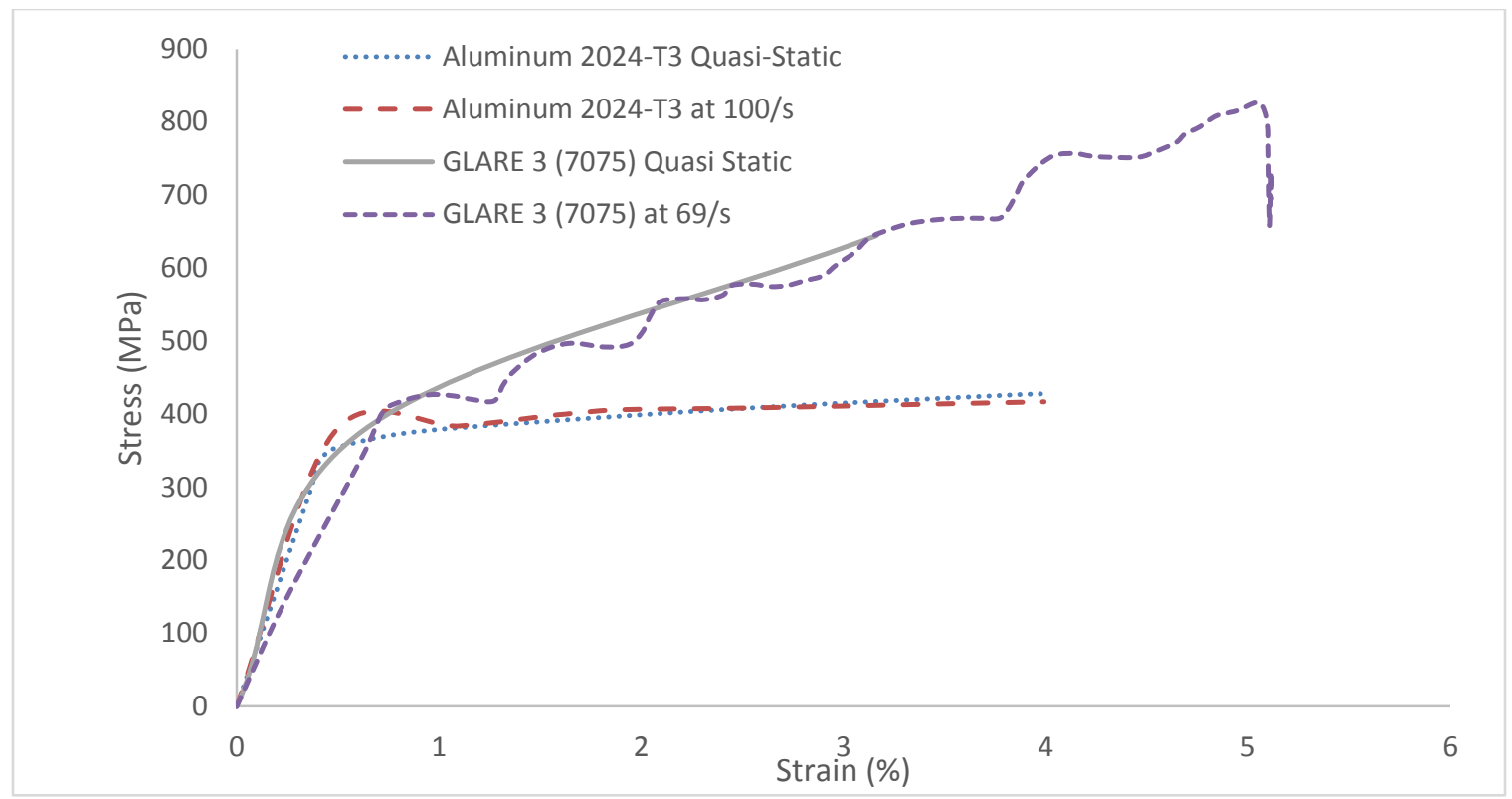

Figure 5: Effect of strain rate on the stress-strain curve of aluminum 2024-T3 (reduced) [26, 33] and GLARE 3 (7075) [32]

At a higher strain rate the available strain energy of GLARE increases resulting in improved impact resistance. Whereas for aluminum 2024-T3 there is a minor increase in yield strength at high strain rate but the post-yield response follows a similar trend to the quasi-static loading rate. Further impact resistance can be gained in FMLs by increasing the volume fraction of metallic sheet at the expense of also increasing the weight [34]. The constituents in FMLs are also being optimized for specific industries as the research work done in references $[35,36]$ has looked at pairing self-reinforced polypropylene with steel and aluminum for the automotive industry. In using a thermoplastic composite in FML technology the impact and vibrational damping advantages of FMLs are paired with the recyclability and fast manufacturing times of a thermoplastic. It has been proven in reference [36] that a self-reinforced aluminum FML can be manufactured with stamp 
forming techniques used by the automotive industry, even surpassing the formability of aluminum in some cases.

\subsection{Impact Testing}

A composite material's response to an impact event is much different than that of a metal. Metallic structures absorb low and intermediate impact energies through elastic and plastic deformation for which permanent deformation usually has a small effect on the load carrying capacity $[37,38]$. Even at very high impact energies that produce penetration of the metallic structure, its load carrying capacity can be predicted from fracture mechanics principles allowing for safe design practices [39]. Fibre reinforced composite structures are restricted in their ability to deform plastically and kinetic impact energy is dissipated through flexure, interlaminar shear deformations, fracture and delamination of the laminate $[29,40,41]$. This ultimately causes large damage areas and results in reduced strength and stiffness [40, 41]. It is therefore very difficult to design for an impact event on a composite structure as damage zones form complex shapes and the predictability of residual properties is poor.

Since a large majority of composite materials tend to behave in a brittle manner in dynamic impact resulting in reduced residual strength, it is crucial that designers understand the damage initiation and growth caused by impacts similar to those in service. While the inclusion of metallic layers in FMLs improves the impact resistance of sandwiched composite layers, the progression of both metallic and composite failure mechanisms during an impact event limits the use of prediction models for designers 
[26]. To better understand the impact performance of composite and FML materials in service a plethora of laboratory impact tests have been performed by researchers over the past forty plus years. This background section will not attempt to cover all aspects of impact testing, but instead will focus on the impact test characteristics relevant to this study. For further information on the impact testing of composite and FML materials the reader is directed to the following review articles [34, 42-45].

\subsubsection{Apparatus}

Charpy and Izod pendulum test rigs were used in early impact testing of thick beam composite specimens that could be ranked based on energy absorption [43]. Due to the complex fracture behaviour of composite materials it was realized that Charpy and Izod impact test methods were inadequate for a realistic impact condition [46, 47]. A major disadvantage of pendulum impact tests was their destructive nature, whereas common impacts in service create barely visible damage states. Based on the need to better control the damage initiated during lab testing, instrumented falling weight devices were constructed to produce more realistic transverse impact scenarios on composite structures. Through instrumentation of the impactor researchers were no longer restricted to solely judging impact resistance on absorbed energy. The force time history can be measured through a load cell in the impactor nose. The load cell starts collecting data from initial contact with the specimen and continues as the impactor traverses through the specimen thickness. The force signal can then be numerically integrated to find the velocity, displacement, and absorbed energy with respect to time for the impact event [26]. Contact force, energy absorbed, and displacement are all recorded parameters 
during an instrumented drop weight event that lend themselves to distinguishing a composite materials impact response. Force-time history has been used specifically to indicate the damage progression in an impacted composite test specimen [48-50]. Figure 6 shows a typical force and energy versus time plot for an impacted specimen for which the characteristic points for post impact analysis are labelled. Incipient load and energy points signify first failure in the laminate through either matrix micro-cracking, fibre damage, or delamination. Maximum load and energy are correlated with the maximum penetration and beginning of rebound for the impactor. The end of rebound phase for the impactor is denoted as the final load and energy points as $P_{t}$ and $E_{t}[51]$.

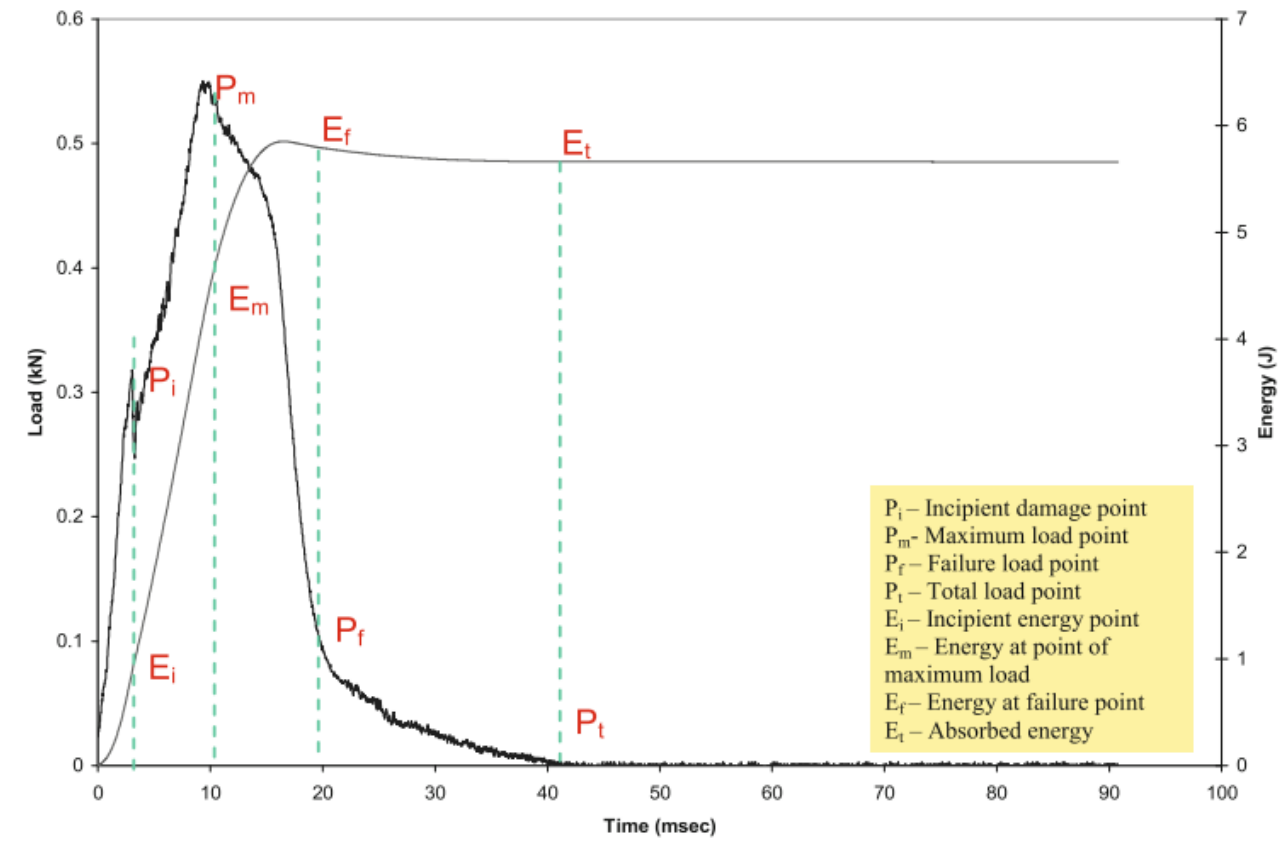

Figure 6: Typical load and energy versus time curve and characteristic points for post impact analysis [44] 
Damage created during instrumented drop weight tests is better controlled as barely visible up to penetration impact damage can be produced through varying the mass, velocity or ultimately the kinetic energy of the impactor.

Few researchers have monitored strains in low-velocity [24] and quasi-static indentation [52] testing to aide in the understanding of a materials impact response. Rosette strain gauges are often employed to capture strains at singular points on the impacted specimen. However, strain histories at the center of the impact specimen have been elusive as the large out-of-plane deformations at the contact site cause the strain gauges to disbond [52]. The incorporation of 3D digital image correlation technology on the non-impacted side of the specimen introduces further methods of evaluating the impact characteristics of a material that common instrumented impact devices cannot. By way of assigning subsets to a high-contrast pattern image of the specimen, DIC technology can track average displacements within subsets during impact events offering accessibility to full field displacement and strain data. Reu et al. [53] has been the only one to implement DIC technology in quasi-static indentation and dynamic impact tests though the tested material was limited to steel plates. An interesting outcome of the experimentation in ref [53] was the need for improved methods of validating DIC strain fields over the use of rosette strain gauges. Others have used DIC in dynamic punch tests to determine the formability of different metallic materials $[36,54,55]$. Therefore there exists a lack of methodology for not only approaching and validating DIC setups for LVI and QSI tests but also in the analysis of full field impact/indent data. 


\subsubsection{Classification}

A general categorization of impact tests is based upon whether the impact is at low, intermediate, high/ballistic, or hyper velocity [44]. It is generally accepted that low velocity impacts are performed with large masses while high velocity impacts are performed with small masses, however there is no clear transition between categories based on kinetic energies or velocities reached [56]. Instead of velocity limits another designation has been suggested in which low velocity impacts (LVI) are defined as impacts where through the thickness fracture or complete penetration of the laminate does not occur [57]. Thus impacts can be defined based on the type of damage incurred, where LVI results in delamination and matrix cracking only [58]. Alternatively, it has been proposed that impact type is governed by the impactor-plate mass ratio and not by impact velocity. It is suggested that if the impactor mass divided by the total plate mass is greater than 2, there will be global deformation of the plate where the contact force and plate deformation will be in phase as shown in Figure 7 a.) [59]. Thus the impact event could be treated as quasi-static as the contact duration is long enough for the entire structure to respond to the impact resulting in more energy being absorbed [60]. When the impactor to test specimen mass ratio is less than $1 / 5$, it means the damage and deformation is restricted to a small area at the impact site and the force and plate deformation are never in phase as shown in Figure 7 b.) [59]. Localized damage in these impact events are the result of stress wave propagation through the material for which the structure does not have time to respond [60]. 


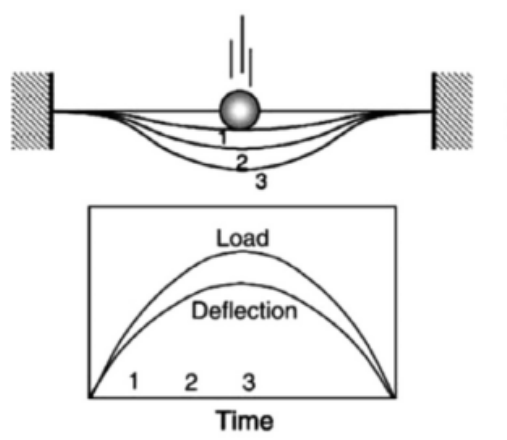

a.)
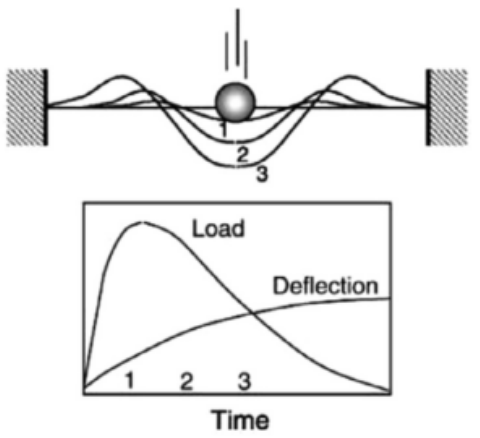

b.)

Figure 7: a.) Global deformation with Quasi-static response b.) dynamic impact response (stress wave) [59]

The quasi-static nature of low-velocity impact tests has allowed researchers to simplify analytical simulation models [26] and mimic dynamic impact behaviour through quasistatic indentation testing.

\subsubsection{Quasi-static Indentation Testing}

A quasi-static indentation testing method offers several advantages to that of low-velocity impact testing including repeatability, lower cost, and better damage control within the specimens [61-63]. The main concern when using QSI tests is their ability to simulate the damage and response parameters of low-velocity impact (LVI) tests. Works that have studied the LVI simulation competency of QSI tests on composite and FML materials are few and include refs $[32,52,61,64]$. Overall, the damage states are found to be similar but force, deflection, and energies can be different depending on the strain rate dependent mechanical properties of the material being tested [32]. Zhou et al. [64] found that even when FML constituents exhibit a low degree of rate sensitivity the impact perforation energy and maximum contact force can be 10\%-15\% higher in LVI tests. 


\subsubsection{Impact Testing for Automotive Applications}

Instrumented drop weight and pendulum impact tests are gaining popularity on the research and development side of the automotive industry as engineers require a detailed account of a materials impact response. While many automotive companies develop inhouse testing standards the majority loosely follow ASTM D3763 when using instrumented impact equipment [65]. Roche and Kakarala [66] were able to determine pertinent impact tests and parameters when considering a wide array of automotive applications in their paper titled Methodology for Selecting Impact Tests of Composite Materials in Automotive Applications. It was determined that four primary impact failure limits existed for automotive components that included incipiency, yield, break, and total failure responses. The belief was that initiation of these failure limits could be easily deciphered from low-velocity impact force-defection plots created with instrumented drop weight equipment (Figure 7). 


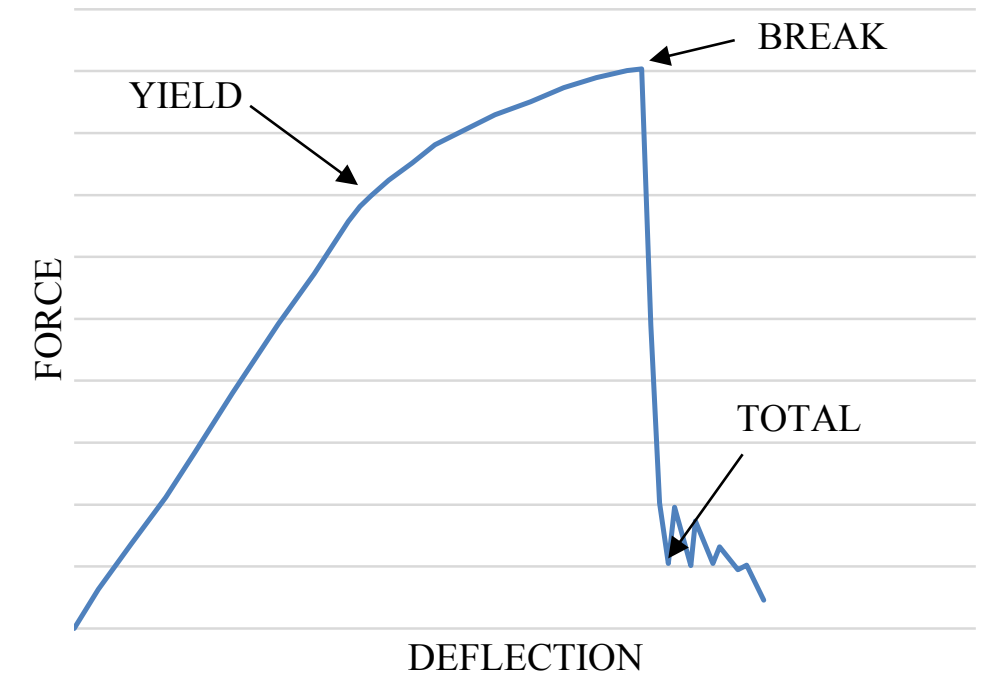

a. )

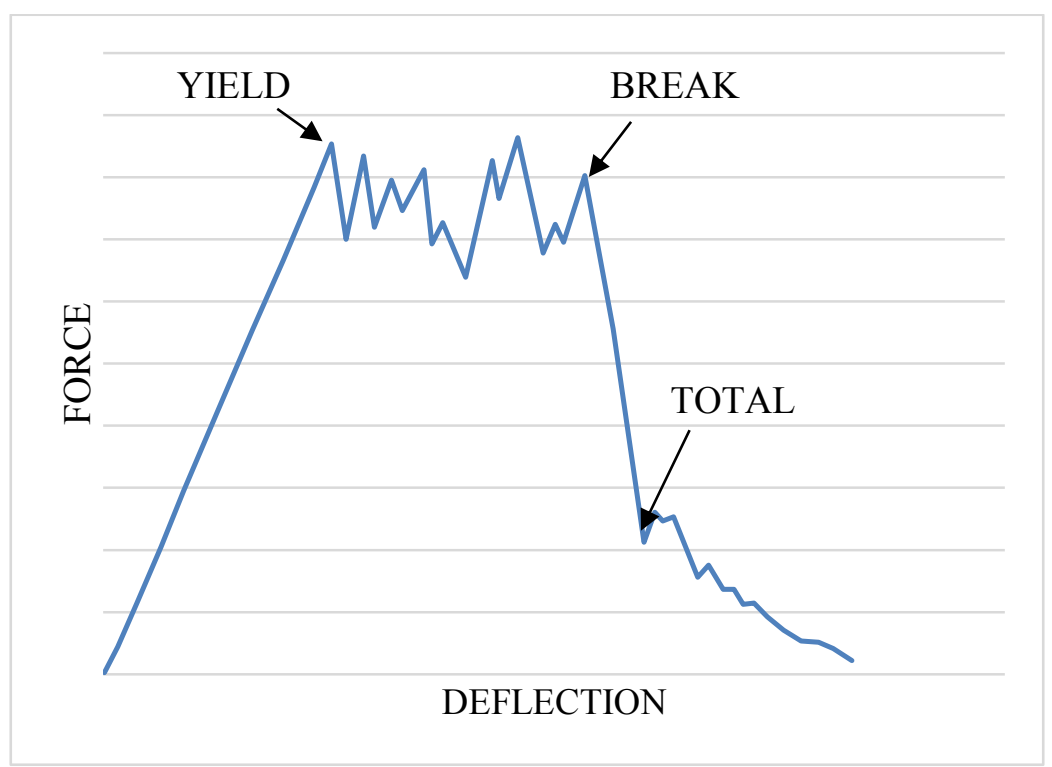

b.)

Figure 7: Typical force-deflection responses from LVI tests of a.) a ductile material and b.) a fibre reinforced material [66]

The exact force, deflection, and energy at the failure limits could then be compared between materials to determine which were best for a specific automotive application. A 
summary of the failure limits, their defining features from a force-deflection plot, and their correlating functional requirements are given in Table 4 .

Table 4: Performance criterion and force-deflection features of impact failure limits [66]

\begin{tabular}{|c|c|c|}
\hline Failure Limit & Performance Criterion & $\begin{array}{l}\text { Defining Force Vs. } \\
\text { Deflection Feature }\end{array}$ \\
\hline Incipiency & $\bullet \begin{array}{l}\text { No property } \\
\text { degradation }\end{array}$ & $\begin{array}{l}\text { Sharp oscillation in } \\
\text { the elastic slope }\end{array}$ \\
\hline Yield & $\bullet$ No visible damage & $\bullet$ Proportional limit \\
\hline Break & $\bullet$ Minimum damage & $\bullet \begin{array}{l}\text { Point at which max } \\
\text { load or near max load } \\
\text { is no longer sustained }\end{array}$ \\
\hline Total & $\bullet \begin{array}{l}\text { Maximum energy } \\
\text { absorption }\end{array}$ & $\begin{array}{l}\text { Point at which } \\
\text { frictional loads are } \\
\text { causing the last } \\
\text { remaining load traces }\end{array}$ \\
\hline
\end{tabular}

After applying the developed methodology to six different automotive applications that included a car fender and an outer door panel the frequencies of interest were obtained (Figure 8) for the individual failure limits and the controlling variables of an impact test. The frequencies in Figure 8 therefore represent the probability that a failure limit and controlling variable will be of importance in the performance and design of an automotive component. 


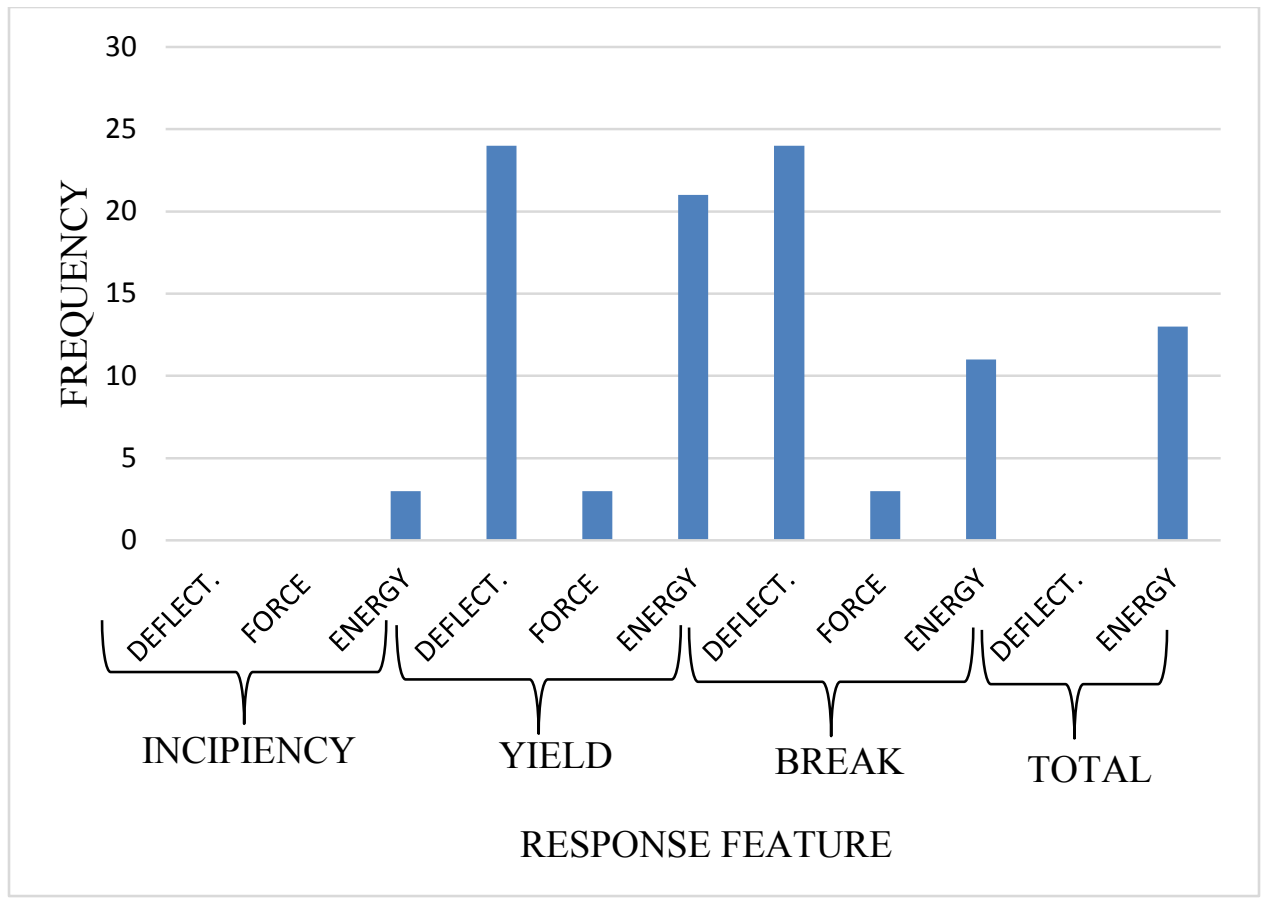

Figure 8: Frequency of occurrence for response features in the characterization of six example applications [66]

It was determined that yield and break responses are more frequently of interest in automotive applications than incipient and total failure limits. It is also worth noting that the ability to absorb deflection is just as important at yield and break as the impact energy to cause the failure limit. Furthermore, the assessment of automotive applications revealed that, at the very least three or more impact test methods with changing stress states would be required to fully evaluate a composite material for an automotive application. However, biaxial bending/tension (ASTM D3763), normal shear, and normal compression impact tests were listed as first priority tests for design. 


\section{Research Scope}

The motivation for this research is based on the need for lightweight materials in the automotive industry and the continuous evolution of testing methodologies used to evaluate their dynamic impact performance. This research therefore bridges the assessment of potential lightweight automotive materials with the evaluation of two testing methodologies used in assessing their low-velocity impact performance. The research objectives of this thesis are:

- To assess lightweight composite and FML materials for automotive applications subjected to dynamic impacts.

- To determine if quasi-static loads can replicate dynamic impact responses and ultimately if QSI tests are an appropriate method to qualify and compare materials for impact prone automotive components.

- To study the advantages of full field DIC data and its capability in characterizing the quasi-static out-of-plane loading of possible automotive materials.

In this research certain limitations in scope were implemented. Only four different variations of lightweight materials were tested, each having approximately the same total thickness of $1.2 \mathrm{~mm}$. These consisted of carbon fibre reinforced thermoset and thermoplastic composites as well as carbon fibre and glass fibre FMLs, all of which were compared to monolithic aluminum 2024-T3. The testing methodologies used for evaluating low-velocity impact performance were restricted to: 1) instrumented drop 
weight low-velocity impact and 2) quasi-static indentation tests. DIC technology was strictly applied to the QSI tests to avoid the need for high speed cameras.

To achieve the first research objective of assessing composite and FML lightweight material for impact performance contact force, central deflection and energy absorption data collected in LVI tests was used. Further evaluation of the FML and aluminum 2024T3 materials was undertaken using full field displacement and strain data gathered in QSI testing. In determining whether quasi-static loads can simulate dynamic impact responses the data collected and damage states resulting from LVI tests were contrasted to that of QSI tests. 


\section{$4 \quad$ Materials and Experimental Procedures}

This chapter provides a detailed overview of the materials and experimental procedures used in this thesis work.

\subsection{Lightweight Materials}

This study investigates the impact resistance of various lightweight composite and FML material options that could replace common steel and aluminum components in the automotive industry. The materials tested range from thermoset and thermoplastic reinforced carbon fibre to two different variations of FMLs, all of which were manufactured at TU Delft. Mechanical properties of the laminate constituents are given in Table 5.

Table 5: Mechanical properties of laminate constituents

\begin{tabular}{|c|c|c|c|c|}
\hline Property & $\begin{array}{c}\text { Aluminum } \\
\text { 2024-T3 [26] }\end{array}$ & $\begin{array}{c}\text { UD } \\
\text { M30SC/DT120 } \\
\text { prepreg [67] }\end{array}$ & $\begin{array}{c}\text { UD S2 } \\
\text { glass/FM94 } \\
\text { prepreg [67] }\end{array}$ & $\begin{array}{c}* \text { Carbon fibre } \\
\text { reinforced } \\
\text { nylon 6,6 [68] }\end{array}$ \\
\hline$E_{11}(\mathrm{GPa})$ & 70.15 & 155 & 48.9 & 24.13 \\
\hline$E_{22}(\mathrm{GPa})$ & 70.15 & 7.8 & 5.5 & $\mathrm{~N} / \mathrm{A}$ \\
\hline$G_{12}(\mathrm{GPa})$ & 27.6 & 5.5 & 5.5 & $\mathrm{~N} / \mathrm{A}$ \\
\hline$\sigma_{\text {ult }}(\mathrm{MPa})$ & 480 & 1800 & 2640 & 193 \\
\hline$v_{12}$ & 0.33 & 0.27 & 0.33 & $\mathrm{~N} / \mathrm{A}$ \\
\hline$\varepsilon_{\text {break }}(\%)$ & $15-18$ & 1.6 & 4.5 & $1.5-2.5$ \\
\hline$\rho\left(\mathrm{g} / \mathrm{cm}^{3}\right)$ & 2.78 & 1.76 & 1.98 & $\mathrm{~N} / \mathrm{A}$ \\
\hline $\mathrm{t}(\mathrm{mm})$ & 0.3 & 0.156 & 0.133 & 0.155 \\
\hline
\end{tabular}

*Property data not available from manufacturer Tencate so reference [68] used. 
Both a glass and carbon fibre reinforced FML were manufactured. The glass FML is designated as GLARE 5-2/1-0.3 which means it has a $\left[0^{\circ} / 90^{\circ} / 90^{\circ} / 0^{\circ}\right] \mathrm{S} 2$-glass arrangement between $0.3 \mathrm{~mm}$ thick clad aluminum 2024-T3 layers where the $0^{\circ}$ direction coincides with the rolling direction of the aluminum. The other FML is denoted as Caral (CARbon Reinforced aluminum) 5-2/1-0.3 which is similar to the GLARE setup except the sandwiched material is carbon fibre reinforced epoxy. Panels of monolithic non-clad aluminum 2024-T3 were also impact tested to gain a full spectrum of composite, FML, and metallic impact samples. A standalone S2 glass reinforced composite panel was not tested in this study due to the large number of test samples.

As a baseline application, aluminum autobody sheets were chosen since they are known to have a standard gauge of 1-1.2 mm [15]. Therefore, all materials tested had a thickness of approximately $1.2 \mathrm{~mm}$ (Table 6 ) to establish comparability of impact results as suggested in ASTM D3763. A complete overview of the laminates tested is given in Table 6 along with their respective layups and physical properties. 
Table 6: Configuration of materials tested

\begin{tabular}{|c|c|c|c|c|c|}
\hline Material & Layup & $\begin{array}{c}\text { Dimensions } \\
(\mathbf{m m} \times \mathbf{~ m m})\end{array}$ & $\begin{array}{c}\text { Panel } \\
\text { Thickness } \\
(\mathbf{m m})\end{array}$ & $\begin{array}{c}\text { Areal } \\
\text { Weight } \\
\left(\frac{\mathrm{kg}}{\mathbf{m}^{2}}\right)\end{array}$ & *MVF \\
\hline Carbon/Epoxy & {$\left[0^{\circ} / 90^{\circ} / 0^{\circ} / 90^{\circ}\right]_{S}$} & $110 \times 110$ & 1.26 & 1.84 & 0 \\
\hline $\begin{array}{c}\text { Carbon/Nylon } \\
6,6\end{array}$ & {$\left[0^{\circ} / 90^{\circ} / 0^{\circ} / 90^{\circ}\right]_{S}$} & $110 \times 110$ & 1.23 & 1.81 & 0 \\
\hline $\begin{array}{c}\text { CARAL } \\
5-2 / 1-0.3\end{array}$ & {$\left[0^{\circ} / 90^{\circ} / 90^{\circ} / 0^{\circ}\right]$} & $110 \times 110$ & 1.23 & 2.6 & 0.49 \\
\hline $\begin{array}{c}\text { GLARE } \\
5-2 / 1-0.3\end{array}$ & {$\left[0^{\circ} / 90^{\circ} / 90^{\circ} / 0^{\circ}\right]$} & $110 \times 110$ & 1.15 & 2.73 & 0.53 \\
\hline $\begin{array}{c}\text { Aluminum } \\
2024-\mathrm{T} 3\end{array}$ & N/A & $110 \times 110$ & 1.22 & 3.42 & 1 \\
\hline
\end{tabular}

*MVF- metal volume fraction

Thermoset based materials were cured in an autoclave while the thermoplastic panel was consolidated in a hot press. Non-destructive inspection tests were performed with TU Delft's C-scanning machine for all composite and FML materials to avoid testing any areas with manufacturing defects. Appendix A provides the $\mathrm{C}$-scan images for all materials manufactured and highlights the defect areas on the as manufactured panels.

\subsection{Low-Velocity Impact Testing}

Low-velocity impact testing was completed on an Instron Dynatup 8200 instrumented drop weight impact tower complimented with Instron Dynatup Impulse impact analysis software at Carleton University. Impact energies can be set by either altering the height of the impactor or by placing individual $1 \mathrm{~kg}$ lead blocks on the impactor carriage.

Velocity of the impactor was captured prior to impact by the passing of a steel velocity 
flag through a magnetic sensor which also triggers a pneumatic braking system on rebound to avoid multiple impacts on each sample. The contact force time history between impactor and sample was measured by a $178 \mathrm{kN}$ load cell. Velocity, displacement and impact energy time histories were then determined by the Impulse software through numerical integration of the force signal. ASTM standard D3763 was used as a guide for analyzing the impact data and for designing the clamping fixture to ensure multiaxial deformation. All samples were cut to square dimensions of 110x110 $\mathrm{mm}$ and clamped between parallel steel plates that had a circular opening of $80 \mathrm{~mm}$ diameter as demonstrated in Figure 9. Eight socket cap bolts held the steel plates together and were tightened to $40 \mathrm{~N} \cdot \mathrm{m}$ with a torque wrench to provide a consistent clamping force.

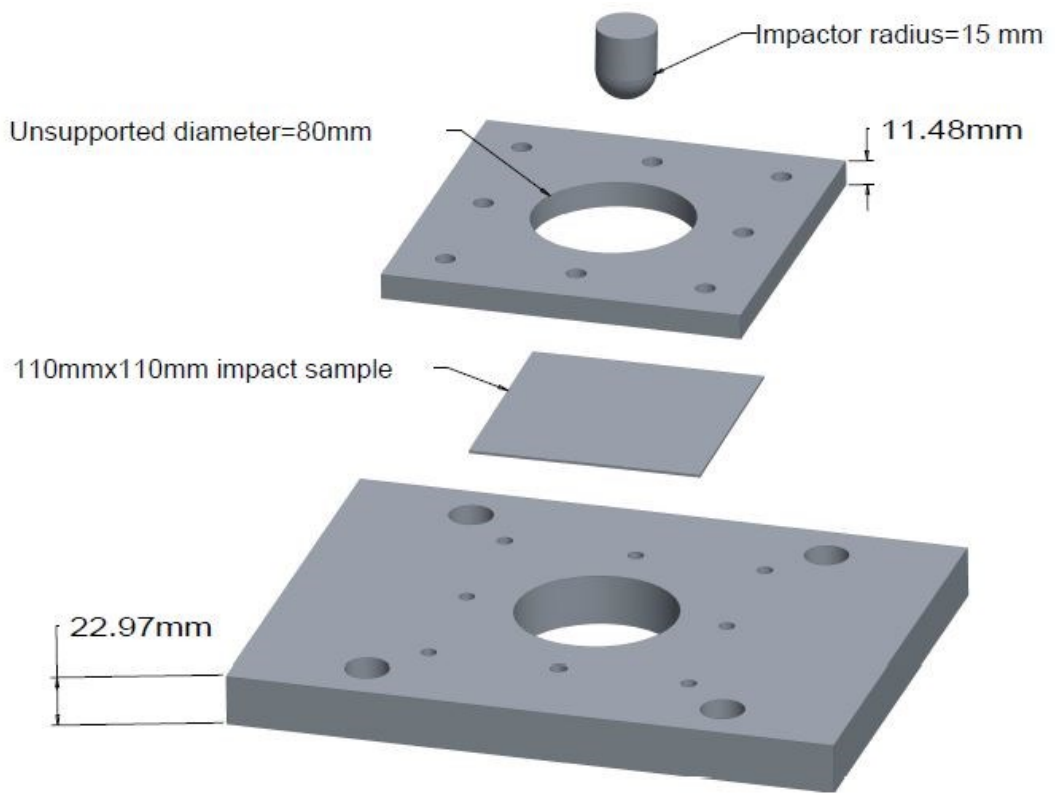

Figure 9: Clamping fixture and impactor for LVI and QSI tests 
A hemispherical impactor with a diameter of $30 \mathrm{~mm}$ (figure 9) and mass of $0.272 \mathrm{~kg}$ was used to impact the lightweight materials considered in this study at the energy levels given in Table 7.

Table 7: Test matrix for LVI tests

\begin{tabular}{|c|c|c|c|c|c|}
\hline & Carbon/Epoxy & Carbon/Nylon & $\begin{array}{c}\text { CARAL 5 } \\
\mathbf{2 / 1 - 0 . 3}\end{array}$ & $\begin{array}{c}\text { GLARE 5 } \\
\mathbf{2 / 1 - 0 . 3}\end{array}$ & Al 2024-T3 \\
\hline \multirow{3}{*}{$\begin{array}{c}\text { Impact } \\
\text { Energies (J) }\end{array}$} & 5 & 5 & 5 & 2 & 2 \\
\cline { 2 - 6 } & 15 & 15 & 12.55 & 5 & 5 \\
\cline { 2 - 6 } & 25 & 25 & 15 & 15 & 15 \\
\cline { 2 - 6 } & 35 & 35 & 25 & 25 & 25 \\
\cline { 2 - 6 } & 45 & 45 & 35 & 35 & 35 \\
\cline { 2 - 6 } & 55 & 55 & 45 & 55 & 55 \\
\cline { 2 - 6 } & & & 55 & 65 & 65 \\
\cline { 2 - 6 } & & & & 75 & 75 \\
\cline { 2 - 6 } & & & & 85 & 85 \\
\cline { 2 - 6 } & & & & 95 & 95 \\
\cline { 2 - 6 } & & & & & 105 \\
\cline { 2 - 6 } & & & & & 125 \\
\cline { 2 - 6 } & & & & & 145 \\
\hline
\end{tabular}

Energy levels were chosen based on achieving a full spectrum of damage progression in each material leading up to full penetration. Each material was impact tested at least once at the energy levels given in Table 7 and in some cases multiple times to establish confidence in the reliability of test results. This is similar to the impact energy methods used in references $[69,70]$. Due to limitations in space for mounting the lead masses, producing impact energies in a 5-155 $\mathrm{J}$ range required altering the impactor height and ultimately the impactor velocities. Thus the potential for strain rate dependent mechanical responses in the materials was unavoidable as impactor velocities exceeded $3 \mathrm{~m} / \mathrm{s}$ [70]. This was accepted as a wide range of velocities was desirable for comparing QSI to LVI 
test parameters. Appendix B provides details on total impactor mass and velocity achieved for each impact energy.

Sources of error for the low-velocity impact tests include any compliance in the custom manufactured clamping fixture (figure 9) which is constructed from mild steel with thickness dimensions based on those from standard Instron dynatup fixtures. To increase the reliability of the LVI data recorded, a larger sample size is encouraged at each energy level in the future. There are no provisions on the ratio of impactor to unsupported region diameter in ASTM D3763 or in reference [66] with regards to impact testing materials for automotive applications. However, the ratio in this study is quite small (ratio is 5.3) which may introduce edge effects around the clamped boundary. Future tests should use larger unsupported regions to minimize this and also better represent automotive applications. The reason for utilizing a relatively large impactor diameter in this study is to induce greater membrane deformation in the samples to better simulate impacts in automotive applications.

\subsection{Aluminum 2024-T3 Low-Velocity Impact Finite Element Model}

Development of efficient and accurate numerical or analytical impact models was not part of this research study. However, a low-velocity finite element model was adopted and slightly modified from reference [52] for aluminum 2024-T3. This allowed the study of strain rates developed at varying impact velocities and to make a general comparison with QSI full field DIC strain data. The finite element software Abaqus/Explicit was used 
with Johnson-Cook flow stress and damage models characterized by the aluminum 2024T3 material parameters in Table 8 .

Table 8: Aluminum 2024-T3 material properties

\begin{tabular}{|c|l|}
\hline Elastic parameters & $\mathrm{E}=70.15 \mathrm{GPa}, v=0.33[26]$ \\
\hline Yield surface parameters & $\mathrm{A}=369 \mathrm{MPa}, \mathrm{B}=684 \mathrm{MPa}, \mathrm{C}=0.015, \mathrm{~m}=1, \mathrm{n}=$ \\
& $0.4[71,72]$ \\
\hline Failure parameters & $d_{1}=0.13, d_{2}=0.13, d_{3}=-1.5, d_{4}=0.011[71,72]$ \\
\hline Fracture energy & $G_{I C}=8 \mathrm{~kJ} / \mathrm{m}^{2}[71,72]$ \\
\hline
\end{tabular}

An R3D4 discrete rigid element impactor head was placed directly on top of a C3D8R solid brick element plate (Figure 10) where mass and initial velocity of the impactor could be specified to match that in LVI tests.

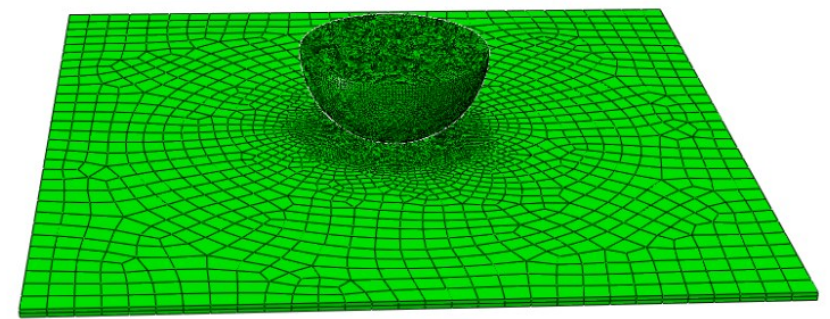

Figure 10: Aluminum 2024-T3 low-velocity impact finite element model

The general contact algorithm specific to Abaqus/Explicit was used to define the interaction between the impactor head and plate. To simulate the clamping of test specimens in LVI tests an encastre boundary condition was placed on the plate surfaces outside the $80 \mathrm{~mm}$ diameter unsupported region. Figure 11 contrasts force-deflection 
plots of aluminum 2024-T3 created in the FEA impact model to those obtained from experimental LVI tests.

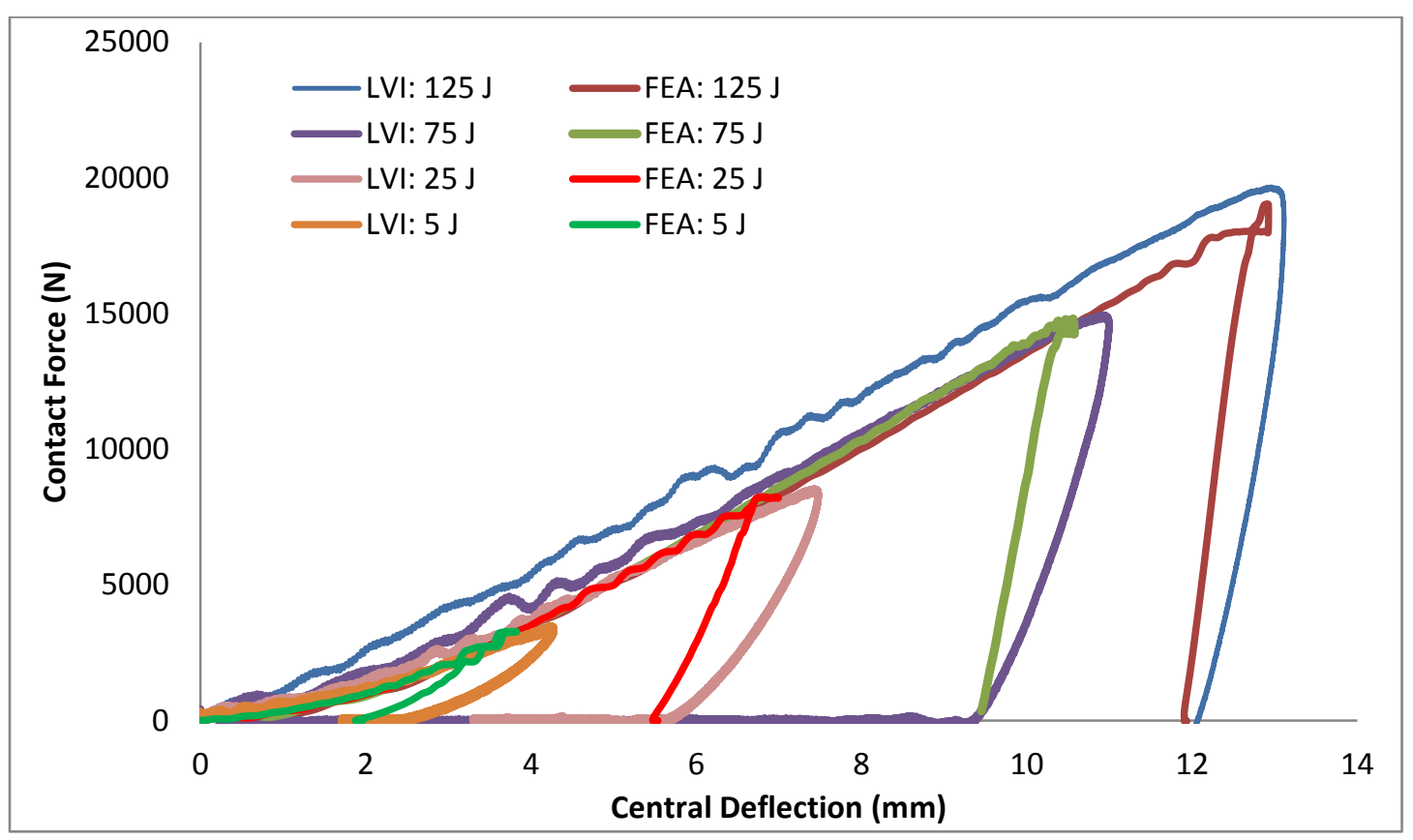

Figure 11: Force-deflection curves of aluminum 2024-T3 from low-velocity impact FEA simulation and experimental LVI tests

Agreement in impact response at various impact energies is good where differences in maximum contact force never exceeds $5 \%$ and differences in central deflection never exceeds $10 \%$ between the FEA model and LVI tests.

\subsection{Quasi-static Indentation Testing}

Quasi-static indentation tests were performed on a $20 \mathrm{kN}$ Zwick testing bench at TU Delft which provided load, point deflection, and energy absorption data. Square 110x110 mm samples of each lightweight material were clamped and indented with an 
identical fixture (Figure 9), clamping force, and indenter to that used for low-velocity impact tests. The indenter head was fixed in position as the sample moved with the Zwick cross-head at a displacement controlled rate of $2 \mathrm{~mm} / \mathrm{min}$ for all tests. Most quasi-static indentation tests were stopped when the force dropped to $20 \%$ of the maximum load or when the tooling came within $5 \mathrm{~mm}$ of separation. This meant the majority of samples were taken to a full puncture damage state. For one round of tests the lightweight materials were only indented to $80 \%$ of their deflection at maximum force which was calculated from previous full indentation tests. This provided samples that were not fully penetrated and could be used to compare damage states with low energy impact samples. Table 9 provides the $80 \%$ deflection value each material was taken to.

Table 9: Set deflection values based on $80 \%$ of deflection at maximum contact force

\begin{tabular}{|l|l|}
\hline Material & $\begin{array}{l}\mathbf{8 0} \text { \% Deflection } \\
(\mathbf{m m})\end{array}$ \\
\hline Carbon/Epoxy & 13.93 \\
\hline Carbon/Nylon 6,6 & 10.71 \\
\hline CARAL 5-2/1-0.3 & 6.5 \\
\hline GLARE 5-2/1-0.3 & 9.15 \\
\hline $\begin{array}{l}\text { Aluminum 2024- } \\
\text { T3 }\end{array}$ & 11.75 \\
\hline
\end{tabular}

Sources of error during QSI tests are similar to those found in LVI testing where there is the possibility of compliance in the test fixture and the small impactor to unsupported region diameter ratio could lead to unwanted edge effects during indentation. Reliability 
is less of an issue in QSI tests however, as each sample was indented to reach puncture at least twice.

A significant portion of this project dealt with the practical issues of implementing DIC technology in a novel mechanical testing platform, in this case quasi-static indentation testing. Due to the lack of methodology available in the literature for approaching and verifying stereovision DIC setups in QSI tests, a complete overview of the process followed in this study is given in chapter 5 . 


\section{$5 \quad$ Methodology for Approaching and Verifying Stereovision}

\section{DIC Setups}

A methodology was developed in this study for approaching and verifying stereovision DIC setups to be used in quasi-static indentation testing. For first time DIC users it is important to understand the terminology and grasp how the parameters associated with this technology can affect the outcome of full field displacement or strain results. Appendix $\mathrm{C}$ provides an overview of the terms associated with DIC and gives quantitative evidence of how DIC parameters can alter experimental outcomes. Initially this chapter will review the MatchID DIC software used. This will be followed by a detailed account of: 1 . choosing the correct speckle pattern, 2. justifying a moving DIC system 3. eliminating shadows, 4. verifying DIC data through displacement rates and a secondary strain measuring technique and 5. monitoring noise and resolutions. The chapter will conclude with a review of DIC quasi-static indentation testing procedures along with a tabulated summary of the DIC testing parameters. Reporting in detail the stated approach towards DIC collected data in QSI experiments should assist researchers in the future when implementing stereovision DIC technology in any mechanical test.

\subsection{MatchID DIC Software}

MatchID DIC software was used on a standalone computing station to capture and save images, perform calibrations, and display full field displacement and strain data. The MatchID software is subset based; meaning the displacement and strains found are 
actually averaged solutions within the center of each subset. To form a new independent solution a subset must move a step size less than half in value of the subset. Furthermore, a subset will only move and deformation fields will only be given in a designated area of interest (AOI). The subset, step size and AOI are shown in Figure 12 which is a sample view of what the stereo DIC system captures for this experiment. It is important that the user identifies the subset and step sizes used for DIC data processing as these parameters effect spatial resolution and strain gradients captured.

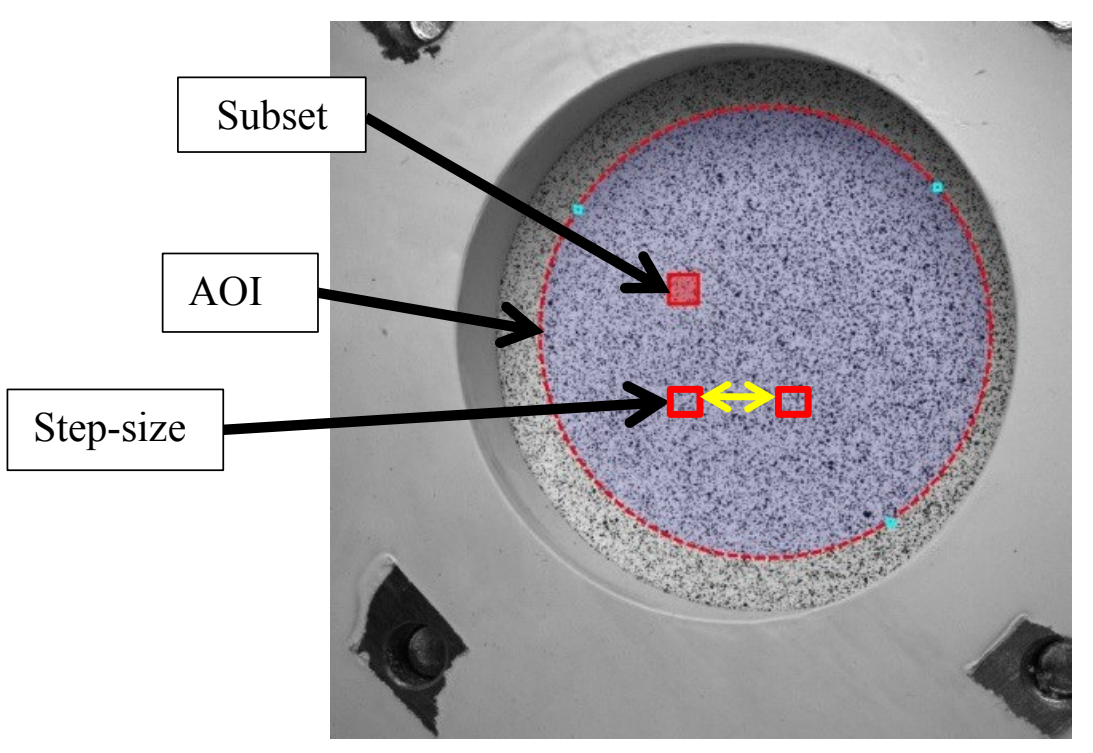

Figure 12: Critical DIC parameters in the MatchID software and how they relate to the captured image [73]

\subsection{Choosing an Appropriate Speckle Pattern}

Achieving high resolutions with DIC means creating good contrast in the loaded objects image as DIC software tracks and compares a contrasted pattern to develop displacement and strain fields. Whether natural or applied, the pattern on the loaded objects surface 
must be random. Applied patterns most often consist of a full coating in white paint followed by black speckling (Figure 12). The quality, density, and size of speckles in a DIC specimen speckle pattern can influence the results through changes in resolutions and noise. Poor quality, dull speckle patterns can cause aliasing while large spaces between speckles can result in poor correlation for small subset sizes. In terms of speckle size, if all speckles are larger than the subset size chosen the DIC software will have a difficult time correlating undeformed and deformed images.

As a first step in approaching the DIC setup, several types of speckle patterns were trialled before conducting the quasi-static indentation tests. The objective was to find a speckle pattern that allowed for suitable subset sizes which could highlight small strain gradients. Available sources for creating speckle patterns were 1.) white and matte black spray paint cans and 2.) Staedtler Fineliner pens ranging in sizes of $0.05,0.1,0.2,0.3$, 0.5 , and $0.8 \mathrm{~mm}$. Spray paint is a popular method used to create speckle patterns as it is fast and easy to apply, yet the pattern will be different each time in speckle density and size. While it is important that the speckle pattern be random and not ordered, it is preferred to have some consistency between samples to make result comparisons more parallel. Thus, during preliminary experiments speckle patterns were applied by hand on the samples with Staedtler pens and with spray paint cans to determine which speckling technique is best suited for the indentation testing. Figure 13 a.) gives an example of the speckle patterns applied by each method. Without comparing the noise and resolution differences it was found that the speckle spacing required and that achievable by hand with pens is the limiting factor. This is easily identified when trying to process a pen 
produced speckle pattern with a subset size of 11 and step size of 5 for which the DIC software is not able to correlate the results due to the large speckle spacing in the pattern. An example of this is in Figure 13 b.) where the $E_{2}$ minimum principal strain fringe plot is shown on an area surrounding the pen speckled area. It is clear that with a small subset size of 11 the DIC software was unable to correlate the deformation leaving much of the strain field missing.
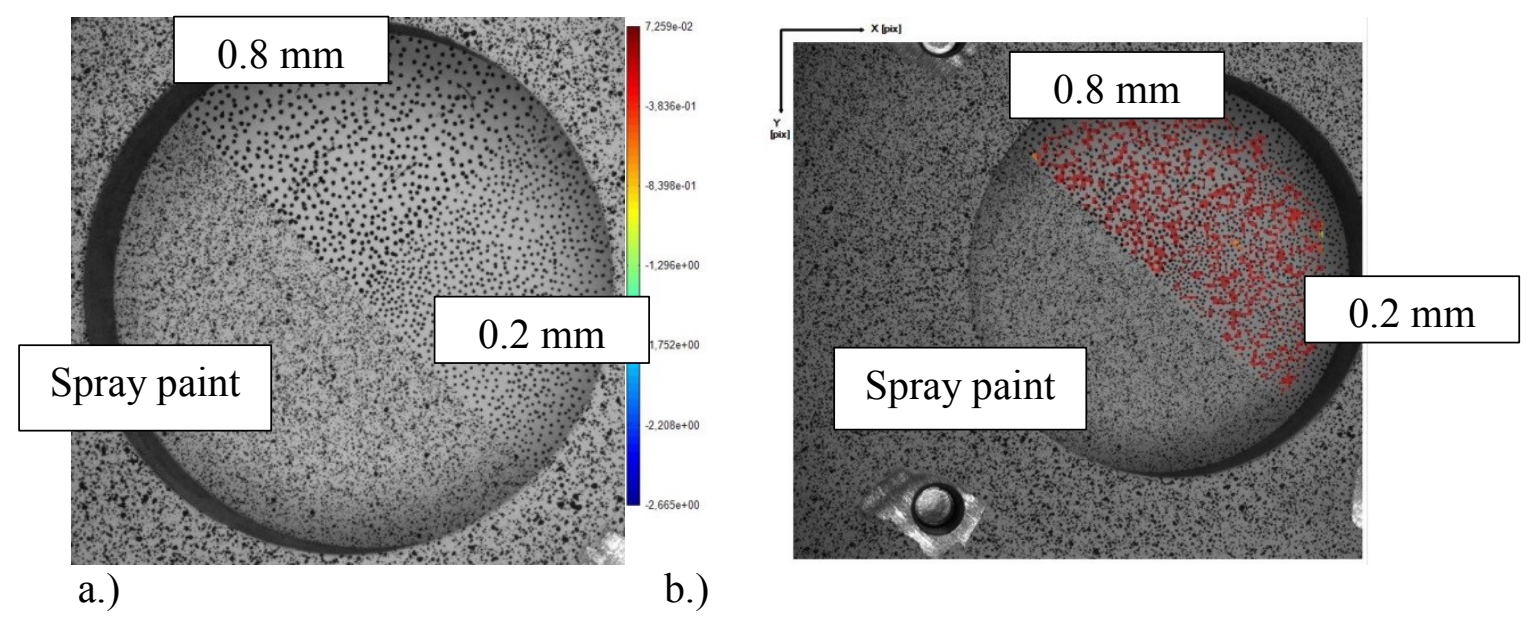

Figure 13: Sample containing three different speckle patterns a.) before indentation b.) minimum principal strain $\mathrm{E}_{2}$ fringe plot captured during indentation

It is suggested that to obtain good correlation when using DIC there must be 3 pixels in a speckle and 3 speckles in a subset [73]. This means the pixel size at the object determines how large a speckle should be and restricts the spatial resolution. For the given DIC experimental setup the pixel to millimeter conversion is $0.0891 \mathrm{~mm}$, meaning an appropriate speckle size should be $0.267 \mathrm{~mm}$ and therefore the pen sizes of $0.05,0.1$, and $0.2 \mathrm{~mm}$ are too small. In terms of spacing the speckles must be $\leq 0.98 \mathrm{~mm}$ apart for a subset size of 11 . 
The speckle patterns created using a pen do correlate well for larger subset sizes such as 21 and 41 as shown in Figure 14.
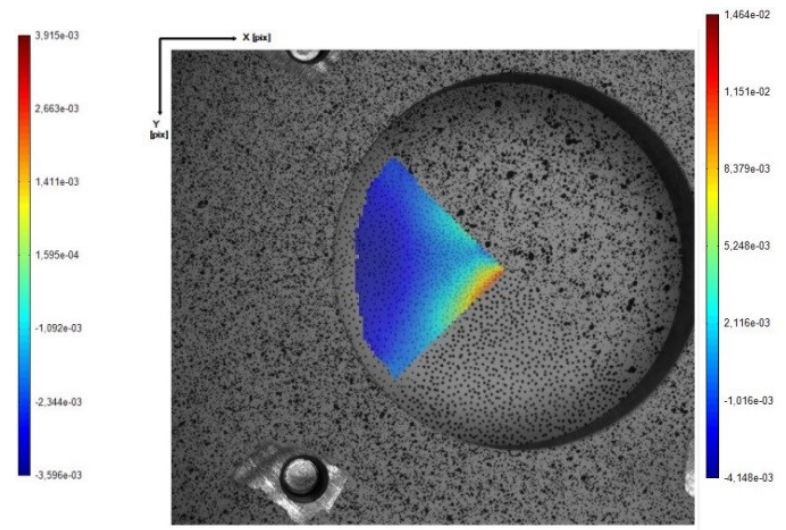

a.)

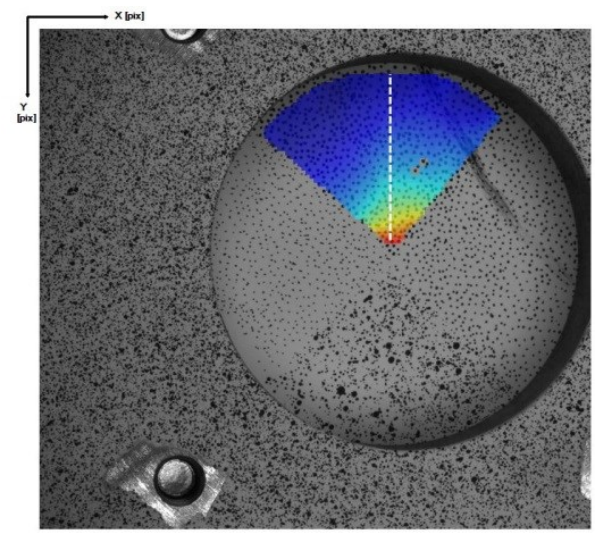

b.)

Figure 14: $\mathrm{E}_{\mathrm{xx}}$ plotted on a.) $0.1 \mathrm{~mm}$ pen speckle pattern for a subset size of 21 and b.) on $0.8 \mathrm{~mm}$ pen speckle pattern for a subset size of 41

However, for the QSI tests performed it is desirable to have flexibility in choosing smaller subsets as a main interest is to look for minute gradients in the strain fields due to failures. Therefore, it was concluded that spray paint is a better speckle pattern producer as it correlated well at smaller subset sizes such as 11 as long as speckle density was high. Figure 15 shows both a poorly correlated spray paint specimen due to low speckle density and an excellent correlation on a properly spray painted speckle sample. 


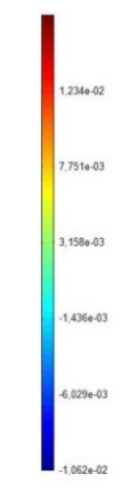

a.)

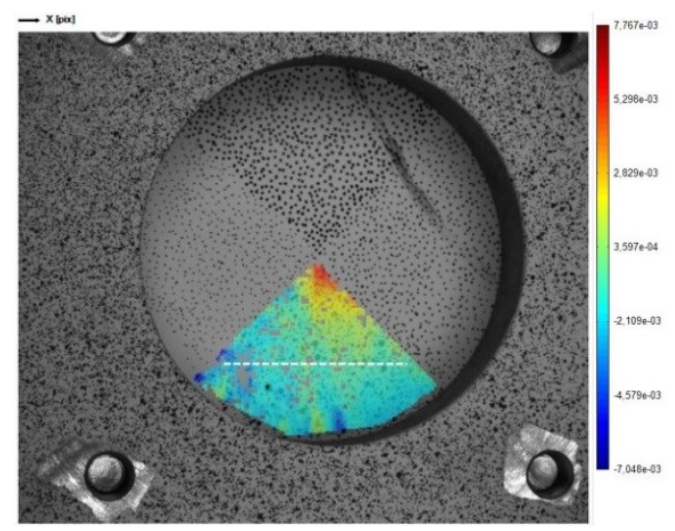

b.)

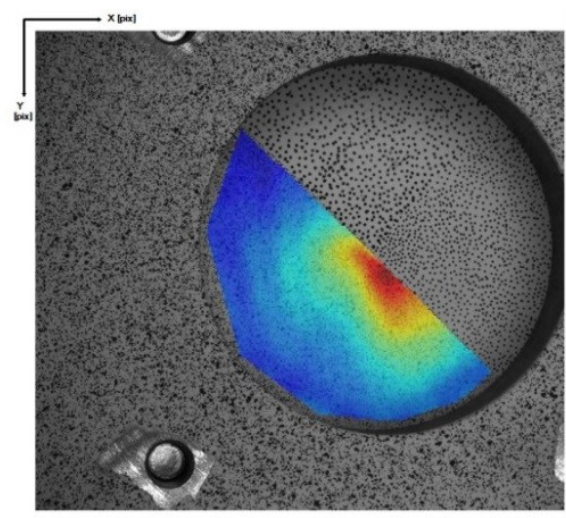

Figure 15: a.) $E_{x x}$ plotted on a spray painted low density speckle pattern for a subset size of 11 b.) $E_{2}$ plotted on a high density speckle pattern for a subset size of 11

The goal for creating spray paint speckle patterns in this study was to emulate as best as possible the speckle pattern shown in Figure 15 b.). A subset size of 11 was chosen for the QSI tests since 11 is the smallest subset size that still provides excellent correlation when using the spray paint can method. Thus the step size was set at 5 since it must be less than half of the subset.

\subsection{Placement of Stereovision DIC Setup}

The stereovision DIC setup included: two Allied Stingray F-125 (1292x964 resolution) cameras with $25 \mathrm{~mm}$ lenses, and two LED flat panel lights. Placement of the DIC equipment was not trivial for the quasi-static indentation experiment as the indenter is stationary while the sample moves with the $20 \mathrm{kN}$ Zwick machine's cross-head. Therefore, two DIC setups were trialled: 1.) the DIC system was stationary on the floor of the lab and the Zwick cross-head moved upwards relative to the cameras and 2.) the 
DIC system was fixed to the Zwick cross-head with aluminum construction rails as displayed in Figure 16 and hence there was no relative motion between the cameras and indented specimen .

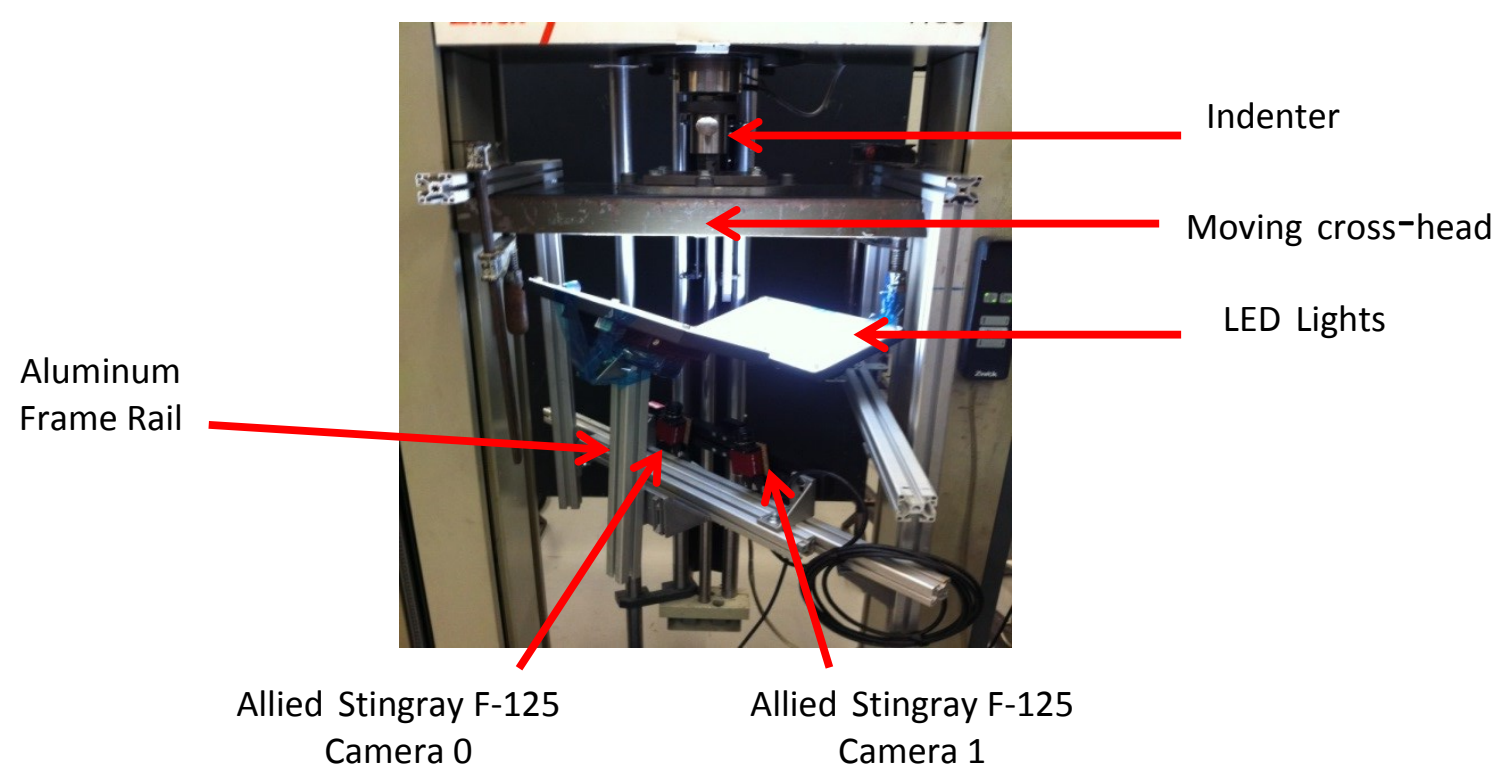

Figure 16: Moving DIC test rig

The selected setup was of that given in Figure 16 since it allows the lighting to move with the specimen and results are easier to interpret since out-of-plane displacement profiles include only the deformation of the specimen and not the motion of the cross-head. A baseline test was performed where the DIC system and cross-head moved $1 \mathrm{~cm}$ over 5 minutes without the indenter coming in contact with the sample to determine if movement of the cameras has a sizeable effect on the DIC measurements. From this baseline test Figures 17 a.) and b.) have been constructed to show variations in displacement and strain resolutions as well as the noise evolution in the cameras as they move $1 \mathrm{~cm}$ in 5 minutes. Each data point in the $\mathrm{x}$-axis represents a picture taken in time 
during the baseline test where the first 4 data points are from static pictures taken before the cross-head moves. The other 5 data points are from pictures taken when the crosshead is moving with a time separation of 60 seconds. Overall the resolutions and noise do not vary significantly due to the motion of the DIC system. However, the displacement and strain resolutions do show an increase near the end of the baseline test which may be due to a change in surrounding lighting as the cross-head approaches the top of the Zwick machine. This is something both setup options would suffer from as shadowing on the specimen becomes worse. Therefore, a moving DIC setup doesn't introduce excess error into the experiment.

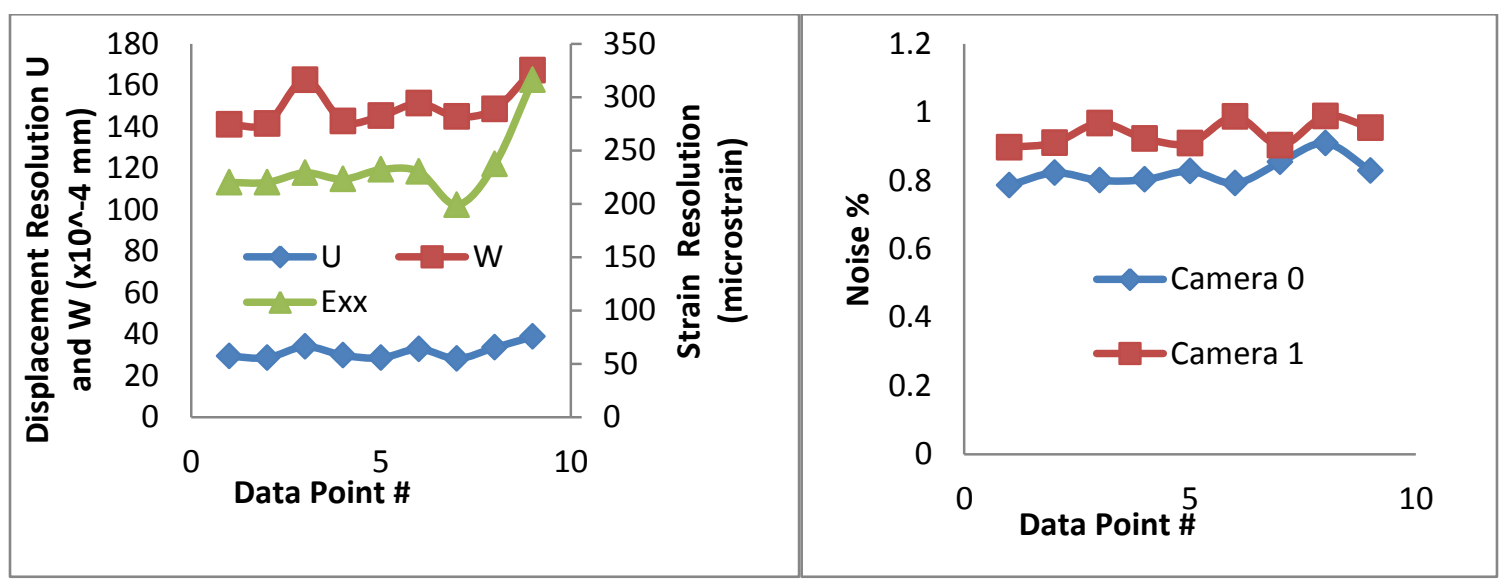

a.)

b.)

Figure 17: a.) change in displacement and strain resolutions and b.) change in noise for a moving DIC setup

\subsection{Lighting and Eliminating Shadows}

Properly lighting DIC samples is a tedious task as an overabundance of light causes 
saturation and not enough results in the formation of shadows. An easy way to evaluate the lighting is to plot the $\mathrm{X}, \mathrm{Y}$, and $\mathrm{Z}$ pixel co-ordinates from a static specimen on a $3 \mathrm{D}$ map. Early on in the QSI tests it was noticed that the out-of-plane pixel positioning does not always project a flat specimen shape as it should. This is demonstrated in Figure 18 where the change in Z-direction pixel position values is shown with a 3D plot of what the DIC system believes the undeformed specimen looks like.

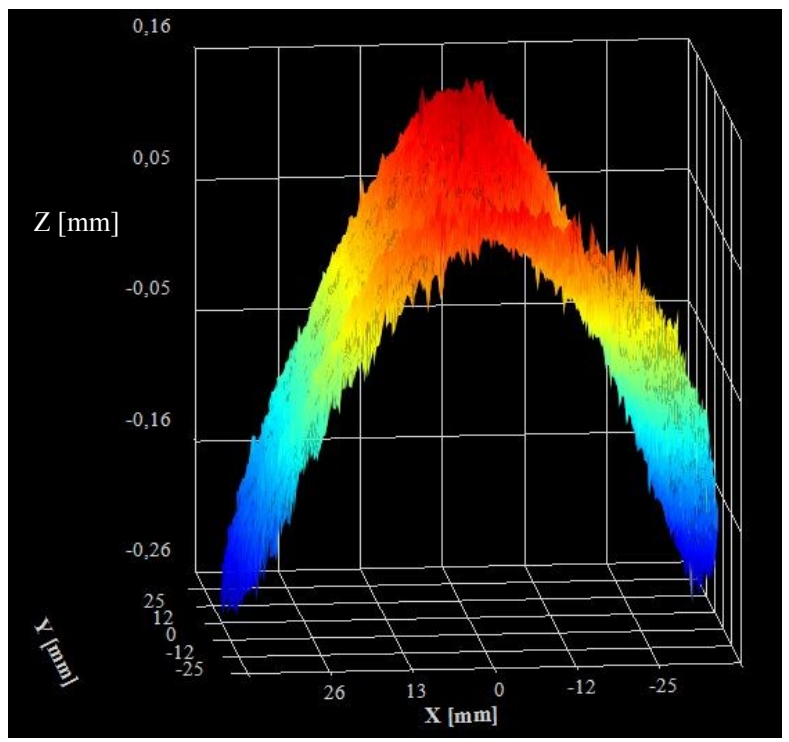

Figure 18: 3D projection of undeformed GLARE specimen

Variations in the out-of-plane pixel positioning may be due to shadowing caused by the large thickness of the test fixture and how the intensity of the light varies from the middle to the outside edges of the specimen. The misconceived deformation can be lowered by adjusting the lighting and painting the test fixture white eliminating as much of the shadowing as possible. Through these techniques the change in out-of-plane pixel positioning across an undeformed specimen (Figure 18) has changed from $1.04 \mathrm{~mm}$ to approximately $0.3 \mathrm{~mm}$. As there is always the possibility of unevenness on panel 
surfaces, undeformed specimens may exhibit deformation indications on 3D projections. The shadowing effect with a bare metal fixture compared to that of a painted white fixture is shown in Figure 19.

a.)

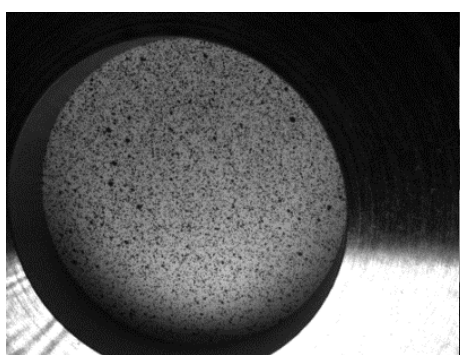

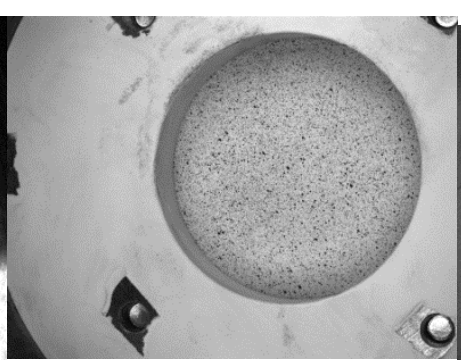

b.)

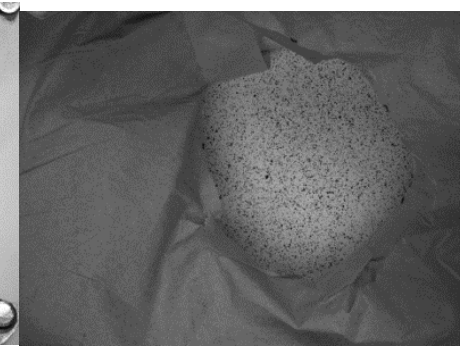

c.)

Figure 19: Shadowing on different test fixture backgrounds a.) bare metal b.) spray painted white c.) covered with opaque cloth

\subsection{Validating DIC Data through Displacement Rates}

Quasi-static indentation tests took place throughout the months of July and August in 2014 with the stereovision DIC setup being constructed and disassembled on several occasions. Therefore, it was important to find a method for validating the full field DIC data after each test was conducted. DIC results can be validated quite easily by comparing the known displacement rate of the Zwick machine $(2 \mathrm{~mm} / \mathrm{min})$ to the calculated out-of-plane displacement rate of the DIC images. To find out-of-plane displacement rates with DIC images one must plot the displacement from the center of the indented sample versus time and find the slope. During the early stages of testing discrepancies were found between the known $2 \mathrm{~mm} / \mathrm{min}$ displacement rate of the machine and the displacement rate calculated through DIC which was consistently found 
to be slower at approximately $1.3 \mathrm{~mm} / \mathrm{min}$. After altering various aspects of the DIC setup it was determined that a larger calibration panel needed to be used in order for the two cameras within the DIC setup to be properly mapped in 3D space. With a stereovision DIC setup it is required to calibrate the MatchID software with a calibration panel consisting of a known pattern of black dots (Figure 20). Once calibrated the MatchID software assigns coordinates to each camera in 3D space allowing for full field in-plane and out-of-plane measurements.

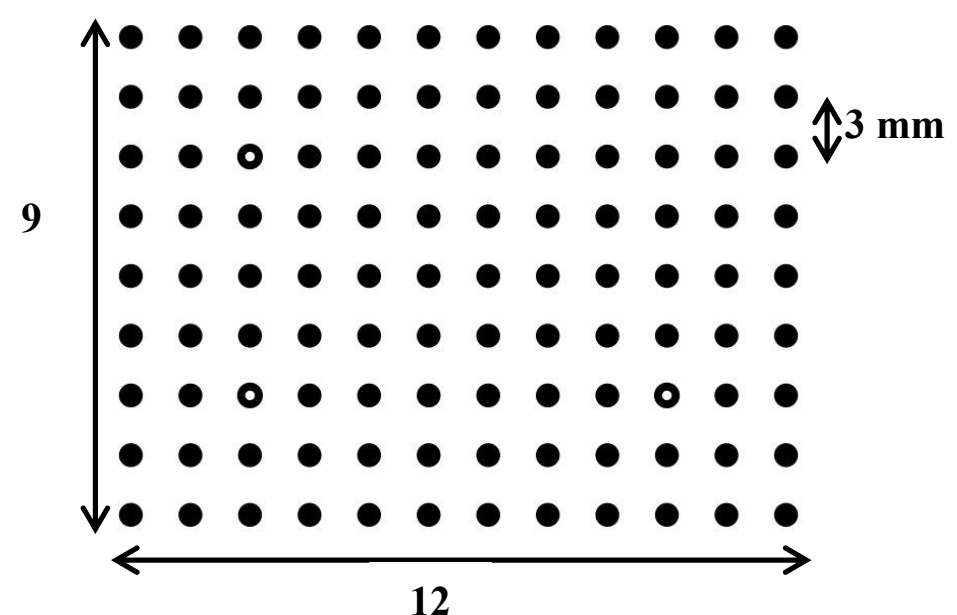

Figure 20: 9x12 DIC calibration panel with $3 \mathrm{~mm}$ grid spacing

Figure 21 highlights the difference between calibrating with a small 9x12 calibration panel with $3 \mathrm{~mm}$ grid spacing and a large $9 \times 9$ calibration panel with $5 \mathrm{~mm}$ grid spacing. Clearly it is important to use a calibration panel of appropriate size since it has a significant influence on the out-of-plane displacement data obtained which is a critical aspect of this research. Therefore, for each quasi-static indentation test completed the DIC out-of-plane displacement rate was calculated as a way to ensure the DIC data was accurate. 


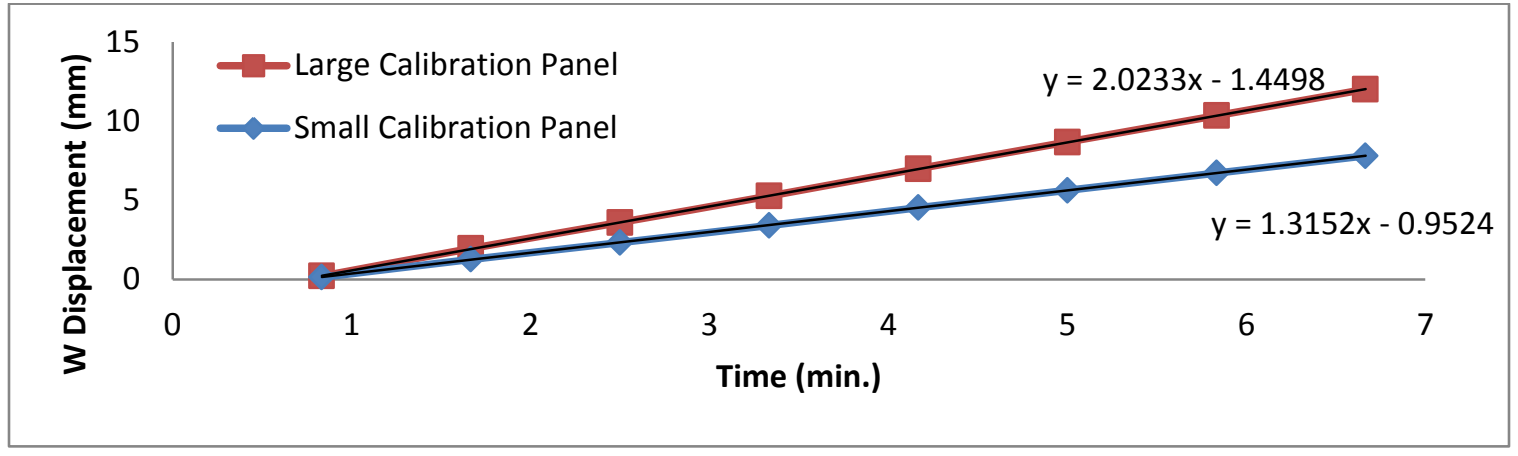

Figure 21: Comparing DIC displacement rates after calibration with different panel sizes

\subsection{Validating DIC Data with a Secondary Strain Measuring}

\section{Technique}

The final validation test conducted on the DIC system included the incorporation of a fibre optic strain sensor with super glue on the non-impact side of a GLARE specimen (Figure 22) to allow for in-plane strain comparisons to be made between the two measuring techniques. After the QSI test was completed it was noted that the fibre optic sensor was still securely mounted to the GLARE panel along its entire length shown in Figure 22. 


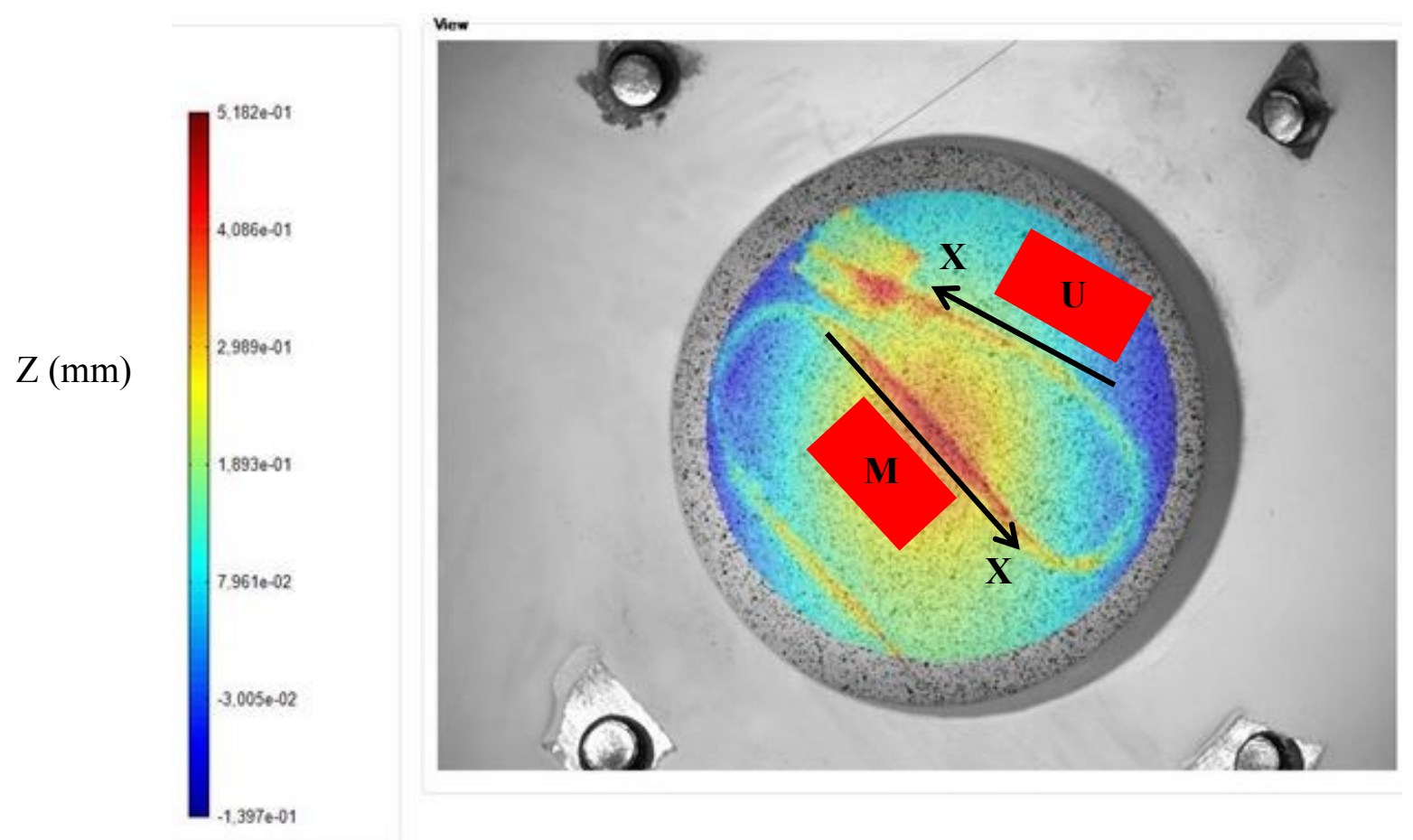

Figure 22: Out-of-plane (Z-direction) position fringe plot showing the fibre optic strain sensor placed throughout GLARE QSI sample

Unfortunately, the Luna ODiSIA fibre optic strain system used is only valid to 10,000 microstrain which was reached within approximately 45 seconds at the tip of the indenter (geometric center) during the quasi-static indentation. Once the limit is reached within the fibre optic strain sensor the data becomes very noisy and unusable. Thus Figure 23 displays the strain comparison between the two systems across section M (Figure 22) of the fibre optic sensor that includes the geometric center of the panel for an indentation time of 20 seconds. 


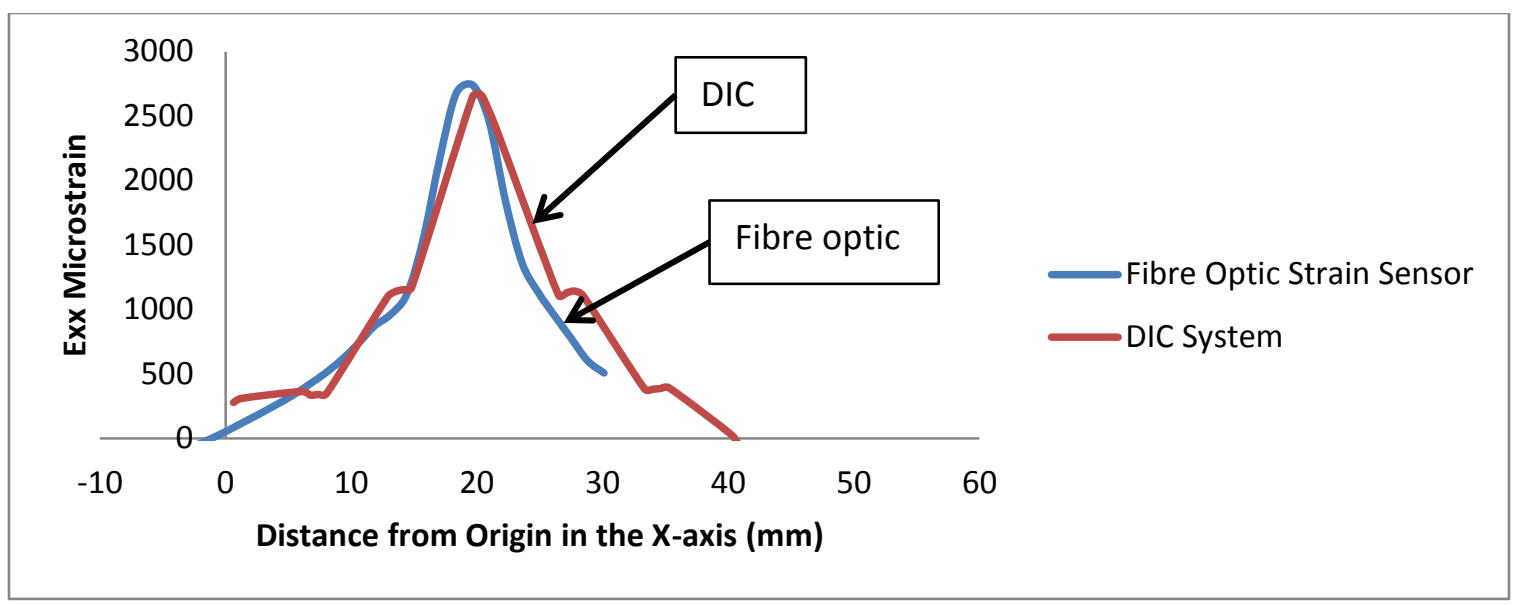

Figure 23: Comparing DIC and fibre optic strain data at an indentation time of 20 (s) for section $\mathrm{M}$ of the fibre optic strain sensor

Strains in the upper portion (section U in Figure 22) of the indented sample are compared in Figure 24 at an indentation time of 260 seconds since strains outside the main indent region do not exceed 10,000 microstrain.

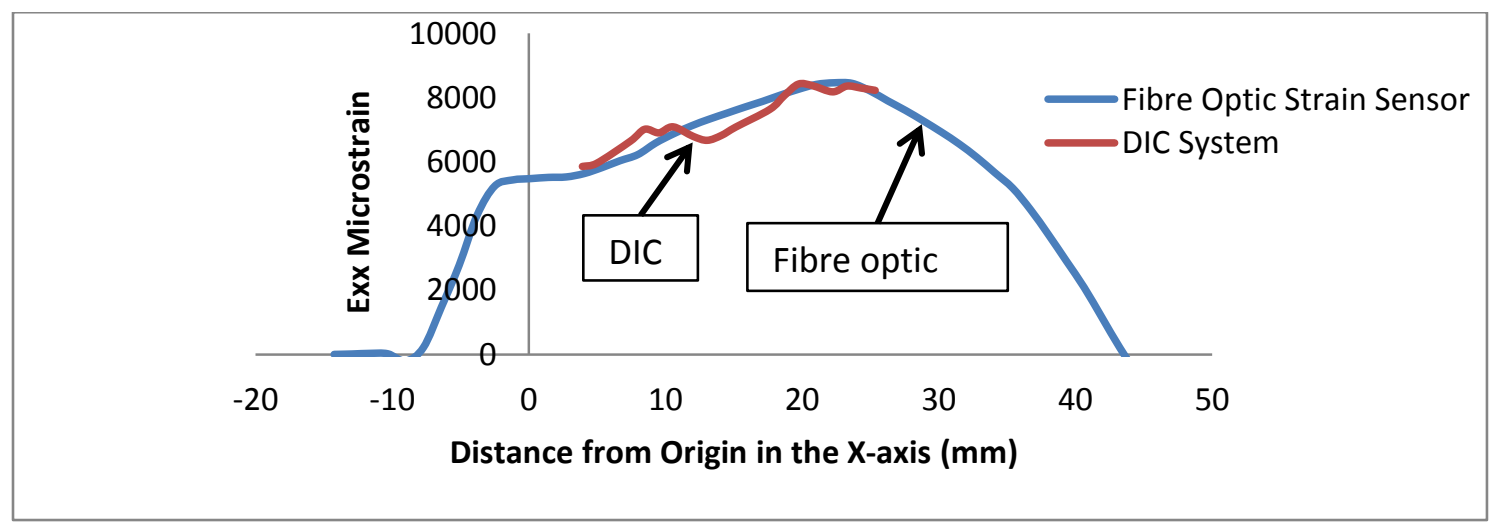

Figure 24: Comparing DIC and fibre optic strain data at an indentation time of 260 (s) for section $U$ of the fibre optic strain sensor

In general the in-plane DIC strains match very well with those from the fibre optic strain 
sensor at varying time durations and position within the specimen. Complete confidence in the stereovision DIC setup and data is therefore achieved. It should be noted that the superposition of the data sets is difficult; however the MatchID software provides an option to conduct transformations on the full field DIC data. This has allowed for placement of a new origin at the start of each fibre optic sensor section as displayed in Figure 22, meaning DIC strains in the $\mathrm{x}$-direction can be aligned along any linear portion of the fibre. Even with these minor difficulties it can be concluded that validating DIC data with a fibre optic strain sensor is a far superior method than using rosette strain gauges as was done in reference [53]. The fibre optic strain sensor can be placed on the same side as the DIC speckle pattern and provides more data points for comparison.

\subsection{Monitoring Noise and Resolutions}

Noise is introduced into the DIC data from the cameras' sensors and digitization of the signal [73]. Quantifying the noise is significant as it has a strong effect on a systems resolution and should thus be checked and recorded for all tests. Noise levels in camera 0 (Figure 16 above) of the stereovision setup are provided in Figure 25 for several indentation tests over the months of July and August, 2014. 


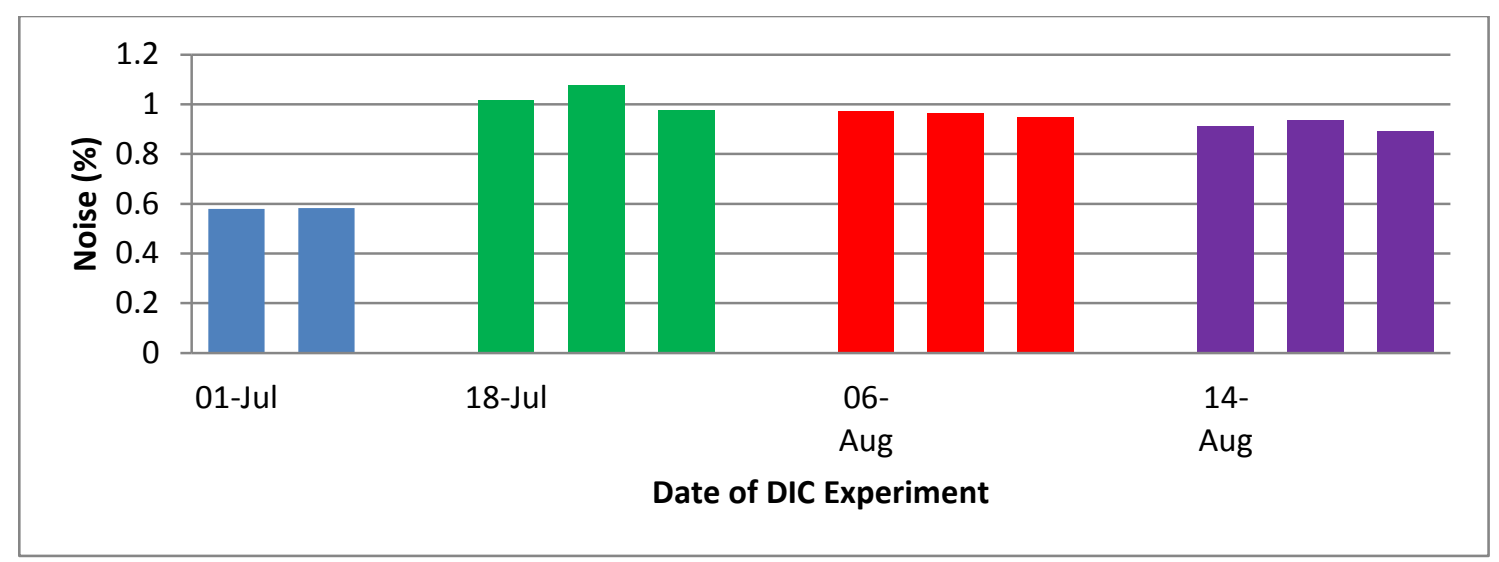

Figure 25: Standard deviation error $\%$ in camera 0

The percent error in camera 0 is shown to have increased after testing on July $1^{\text {st }}$ perhaps due to changing of the bare metallic fixture surface to white to reduce shadowing. A blue opaque shop towel was used to cover the white test fixture (Figure 19 c.)) to determine if the state of the test fixtures surface affected camera noise. This comparison is given in Figure 26 where it is shown that the darker test fixture background produces less noise than the one painted white. From Figure $\mathrm{C} 1$ in Appendix $\mathrm{C}$ it is shown that in-plane displacement resolutions increase linearly with camera noise. Therefore doubling the noise levels from $0.5 \%$ on July 1 to $1 \%$ on July 18 , the in-plane displacement resolution approximately doubles from $16.83 \times 10^{-4} \mathrm{~mm}$ to $33.96 \times 10^{-4} \mathrm{~mm}$ based on Figure 65 . 


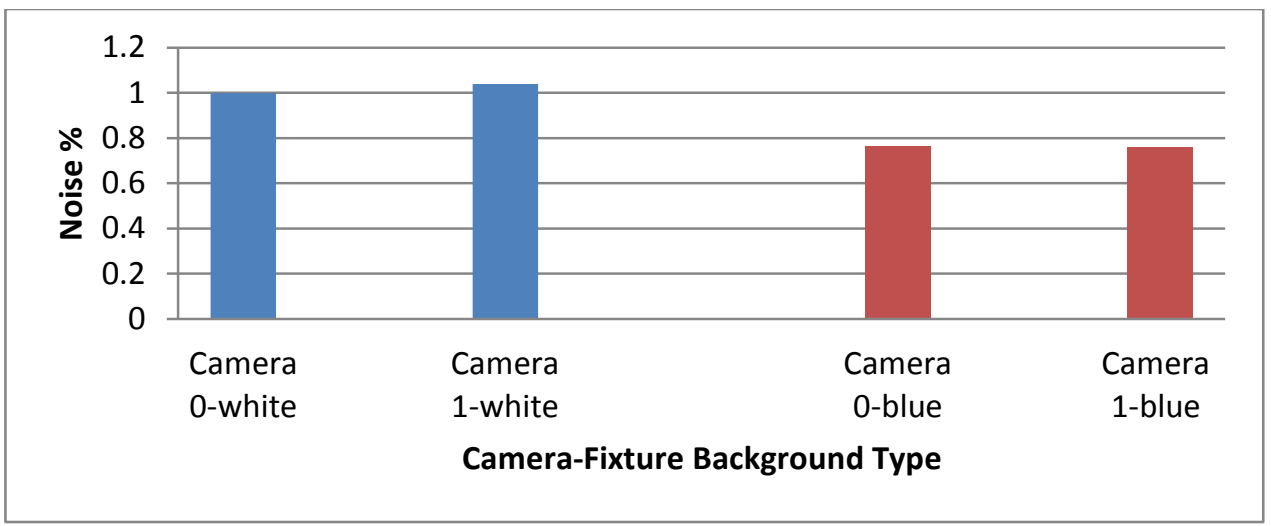

Figure 26: Comparison of camera noise with changing test fixture surface

As with noise, displacement and strain resolutions have been gathered and compared over the testing months of July and August, 2014 to ensure the stereovision DIC setup is consistent. Figure 27 shows how the out-of-plane displacement and strain resolutions have varied throughout testing.

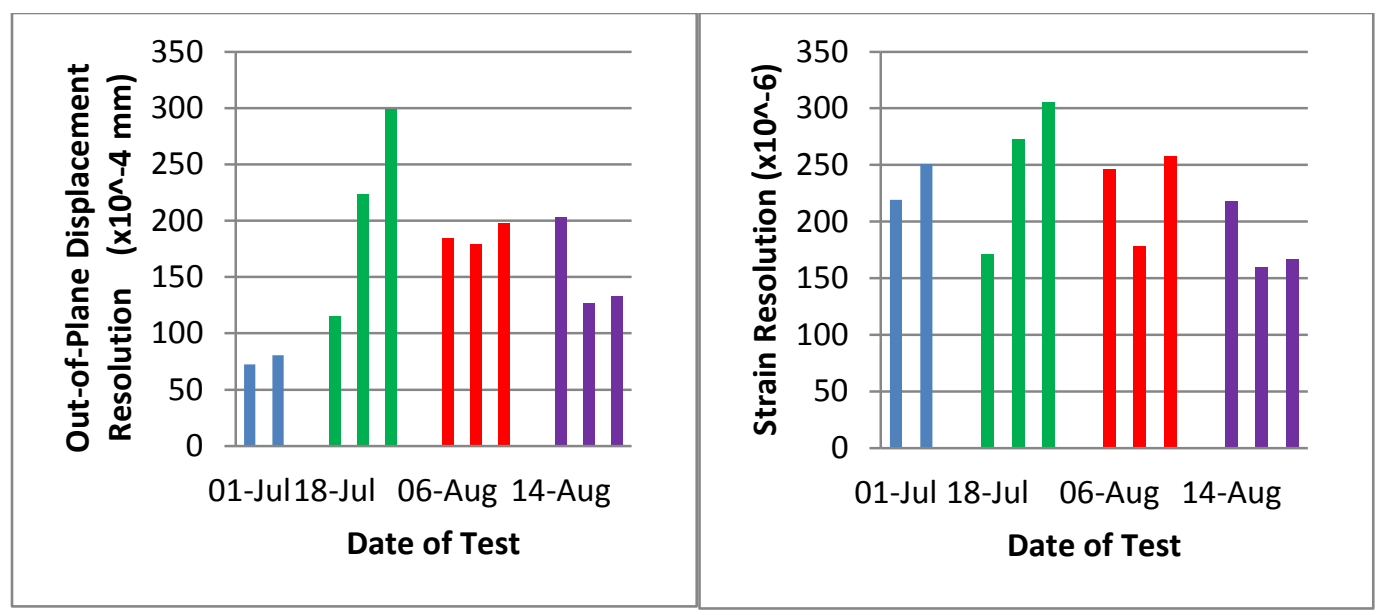

a.)

b.)

Figure 27: DIC resolutions a.) out-of-plane displacement b.) in-plane strain 
As expected the out-of-plane displacement resolutions have increased based on the increase in camera noise after the July $1^{\text {st }}$ test, yet the strain resolutions are reasonably consistent. Although painting the test fixture white has affected the out-of-plane displacement resolutions negatively it should be kept in mind that the resolutions are gathered from static images of undeformed samples. Hence, when the indentation sample experiences large out-of-plane deformations the shadowing on the sample becomes more severe affecting the resolution quality. It was therefore decided to keep the test fixture painted white, but for future tests perhaps only painting the areas closest to the indent sample will suffice.

\subsection{DIC Testing Procedure and Summary of Parameters}

At the start of a quasi-static indentation test each day, the stereovision DIC setup underwent calibration only once using a 9x9 (dot pattern) calibration panel with $5 \mathrm{~mm}$ spacing between calibration dots. Prior to an individual test, forty static images of the specimen were taken at a one second interval to determine noise and resolution characteristics of the setup. To begin the actual indentation test the $20 \mathrm{kN}$ Zwick machine and MatchID image grabber were started simultaneously with images from each camera being saved at one second intervals. After reaching full penetration of the material the MatchID image grabber was stopped and the DIC data was verified through displacement rates. Processing DIC results involves reporting set and measured parameters such as noise and spatial resolution that may have an effect on displacement and strain results. Important parameters set in the MatchID software and averaged noise and resolutions are provided in Table 10 . 
Table 10: Summary of DIC parameters for QSI tests

\begin{tabular}{|c|c|}
\hline Technique Used & $\begin{array}{l}\text { Stereovision Digital Image } \\
\text { Correlation }\end{array}$ \\
\hline Noise Camera 0 & 0.9989 \\
\hline Noise Camera 1 & 1.0211 \\
\hline Pre-filtering & Gaussian - Kernel 5 \\
\hline Subset & 11 \\
\hline Step & 5 \\
\hline Correlation Criterion & ZNSSD \\
\hline Shape Function & Affine \\
\hline Interpolation Function & Polynomial - Bicubic \\
\hline Measurement Points & $\mathrm{N} / \mathrm{A}$ \\
\hline Image Capture Rate (pics/second) & 1 \\
\hline Displacement: & \\
\hline Spatial Resolution & 11 pixels \\
\hline In-plane resolution & $35.1 \times 10^{-4} \mathrm{~mm}$ \\
\hline Out-of-plane resolution & $175.25 \times 10^{-4} \mathrm{~mm}$ \\
\hline Strain: & \\
\hline Smoothing Method & Polynomial - bilinear \\
\hline VSG & $6.318 \mathrm{~mm}$ (71 pixels) \\
\hline Spatial Resolution & $7.208 \mathrm{~mm}$ (81 pixels) \\
\hline Resolution & $216 \mu \epsilon$ \\
\hline
\end{tabular}


Even with the standard approach and validation of the stereovision DIC setup there exists potential sources of error. During QSI testing the change in surrounding lighting in the laboratory was unavoidable and could have potentially caused unwanted shadowing on the specimen. Thus in future work the stereovision DIC system should be re-calibrated every thirty minutes to one hour following re-adjustments of the LED light intensities. Furthermore, during the QSI tests out-of-plane displacements became quite large and may have affected the correlation between stereoscopic camera pairs. To avoid this in the future a four camera DIC system could be used. Lastly, quantification of vibrations in the cameras during puncture of an indented sample should be studied to give further confidence in the moving DIC system. 


\section{$6 \quad$ Results and Discussion}

\subsection{Low-velocity Impact Testing}

One of the objectives of this research is to correlate low-velocity impact tests performed with the impact requirements of automotive applications. A biaxial bending/tension impact testing method has been chosen to rate the impact resistance of lightweight materials in this study since it creates stress states that are of high design priority [66]. The materials in this study are therefore not being considered for a specific automotive application as this would require the implementation of multiple impact testing methods. Instead the materials are being evaluated under a definitive impact condition that applies to numerous automotive components. Roche and Kakarala's [66] impact characterization of six different automotive applications will be used as a guide towards determining what failure limits with corresponding controlling variables are of interest for the impact testing method chosen. Throughout the section comments on the limitations affiliated with instrumented drop weight testing data in the assessment of potential automotive materials will be provided.

\subsubsection{Identifying Failure Limits}

According to Roche and Kakarala [66] failure limits are to be defined from forcedeflection curves developed at the low-velocity impact energy near or at puncture as depicted in Figure 28 for the materials tested. 


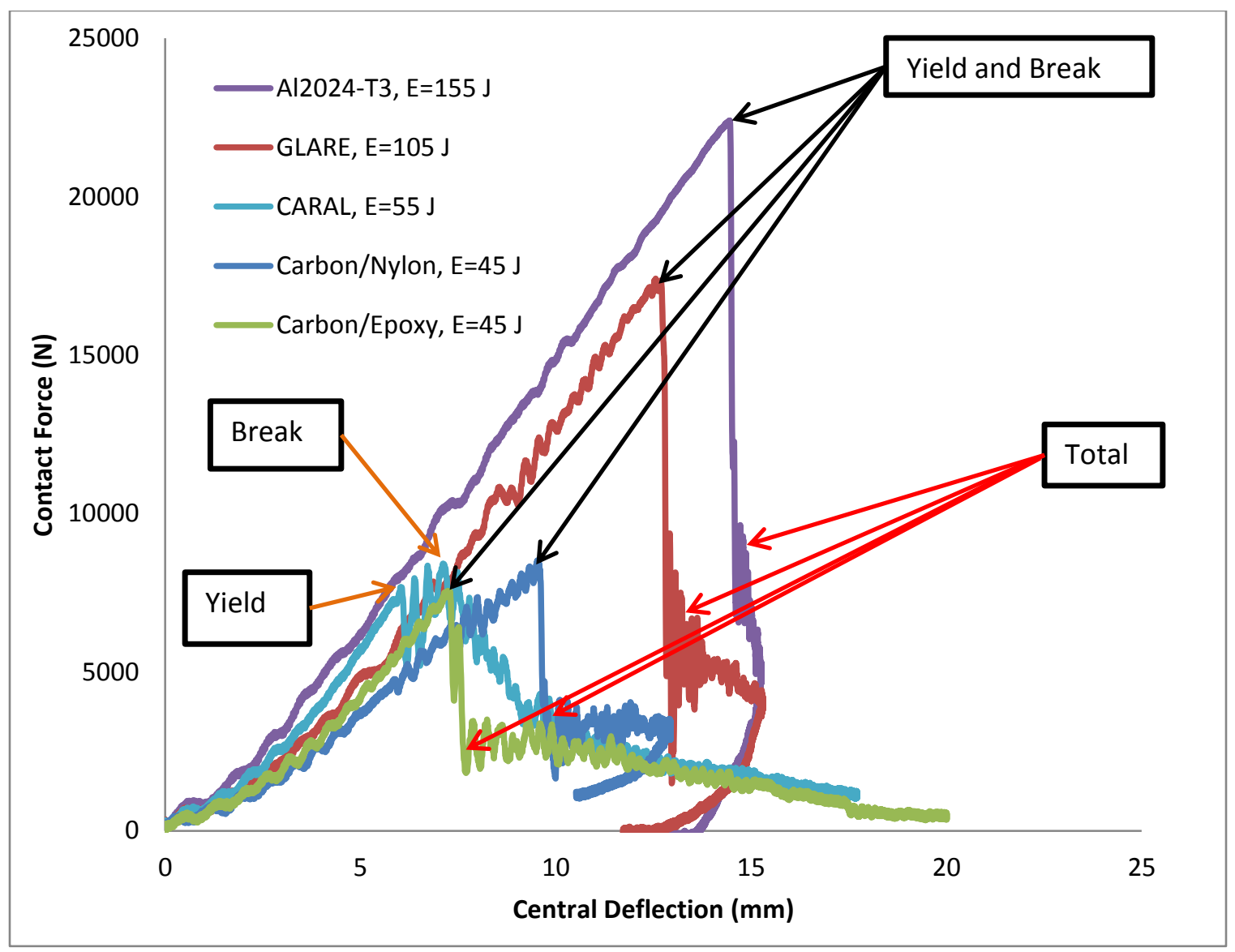

Figure 28: Force-deflection plots from LVI tests highlighting failure limits

Total and break responses are well defined from the force-deflection curve, however incipiency and yield are less obvious. Incipiency, defined as early property degradation, is difficult to differentiate from early vibrations as the impactor strikes the plate (Figure 28). Since incipiency is of little importance in automotive applications it is no longer considered here. The yield failure limit is defined as the proportional limit and is shown to be indistinguishable from the break response for the majority of lightweight materials tested. This can be attributed to the small thickness chosen for the specimens where membrane deformation and fast fracture are promoted versus delayed damage regions found in impact testing thick panels [43]. CARAL is the only material that has a 
definitive yield response where break exceeds the proportional limit. Roche and Kakarala [66] associate the yield failure limit with the formation of visible damage in the impact specimen, however this does not coincide with the proportional limit in the forcedeflection plot. Visible damage was present in each material at impact energy levels well below that to cause the proportional limit or break responses. The first occurrence of visible damage on the non-impact side is shown in Figure 29 for the carbon composite panels in the form of fibre breakage and matrix cracking. For comparison the impact energy required to obtain the visible damage and to reach the proportional limit is given.

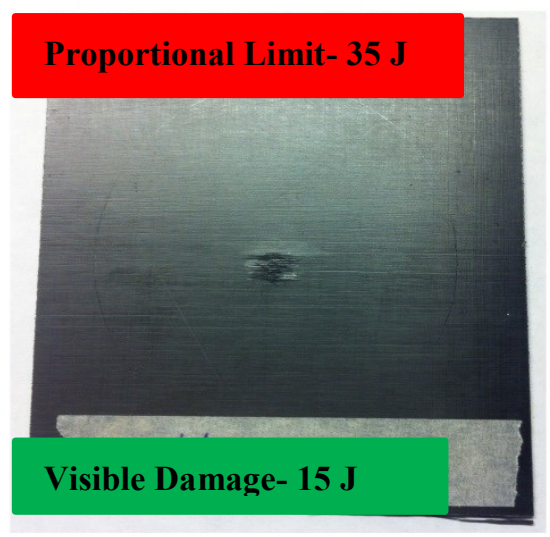

a.)

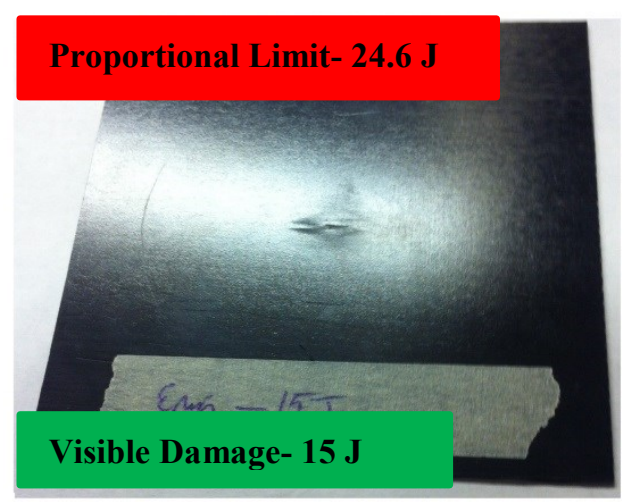

b.)

Figure 29: Visible damage states on the non-impact side that occur before the proportional limit is reached for a.) carbon/nylon and b.) carbon/epoxy

In the FML and monolithic aluminum 2024-T3 panels damage progresses from a small plasticity zone at the site of impact to plastic deformation of the entire $80 \mathrm{~mm}$ diameter unsupported region. Unfortunately, neither of the plastic damage modes is definable in the force-deflection plots in Figure 28. The final damage state before full puncture takes 
place is cracking of the aluminum layer on the non-impact side for FML samples or complete through thickness cracking in the monolithic aluminum 2024-T3. Cracking damage modes take place at approximately the break point in both FML and aluminum 2024-T3 materials. Examples of plasticity and visible damage occurring at lower impact energies than the proportional limit are given in Figure 30.
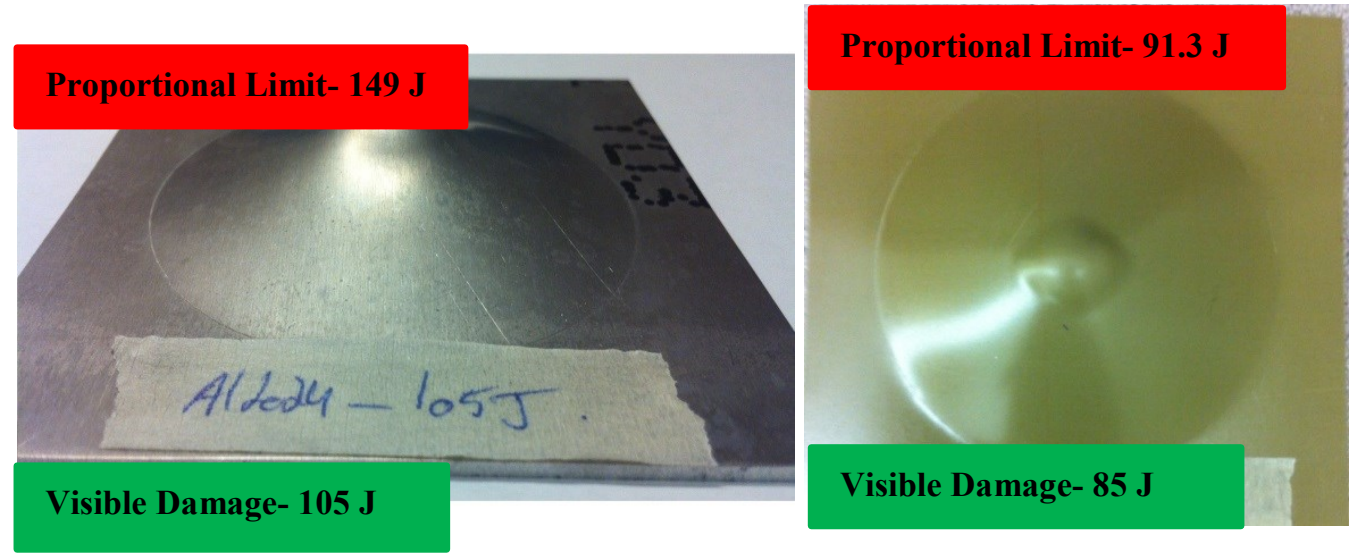

a.)

b.)

Figure 30: Plastic deformation at impact site and in unsupported region of a.) aluminum 2024-T3 panel and b.) GLARE panel

It is then proven that the start of visible damage cannot be deciphered from a forcedeflection curve and this failure limit must then be based on inspecting the impacted samples at each energy level. From here on this failure limit will be referred to as visible damage instead of yield.

\subsubsection{Visible Damage Failure Limit}

The importance of the visible damage failure limit is based on the need to maintain an aesthetically pleasing structure for the automobile consumer. Visible damage also 
indicates that structural integrity may be compromised. Automotive components are thus required to sustain low energy impacts during manufacturing and in service without incurring visible damage. Whether this visible damage is relevant on the impacted, nonimpacted or both sides of the structure is dependent on the specific application. For example, in a vehicle fender visible damage on the side of impact is of concern as it is viewed in the form of a permanent dent.

Deflection at maximum load and permanent dent depths obtained from the Impulse impact analysis software are given in Figure 31 at low impact energy levels for the lightweight materials tested.

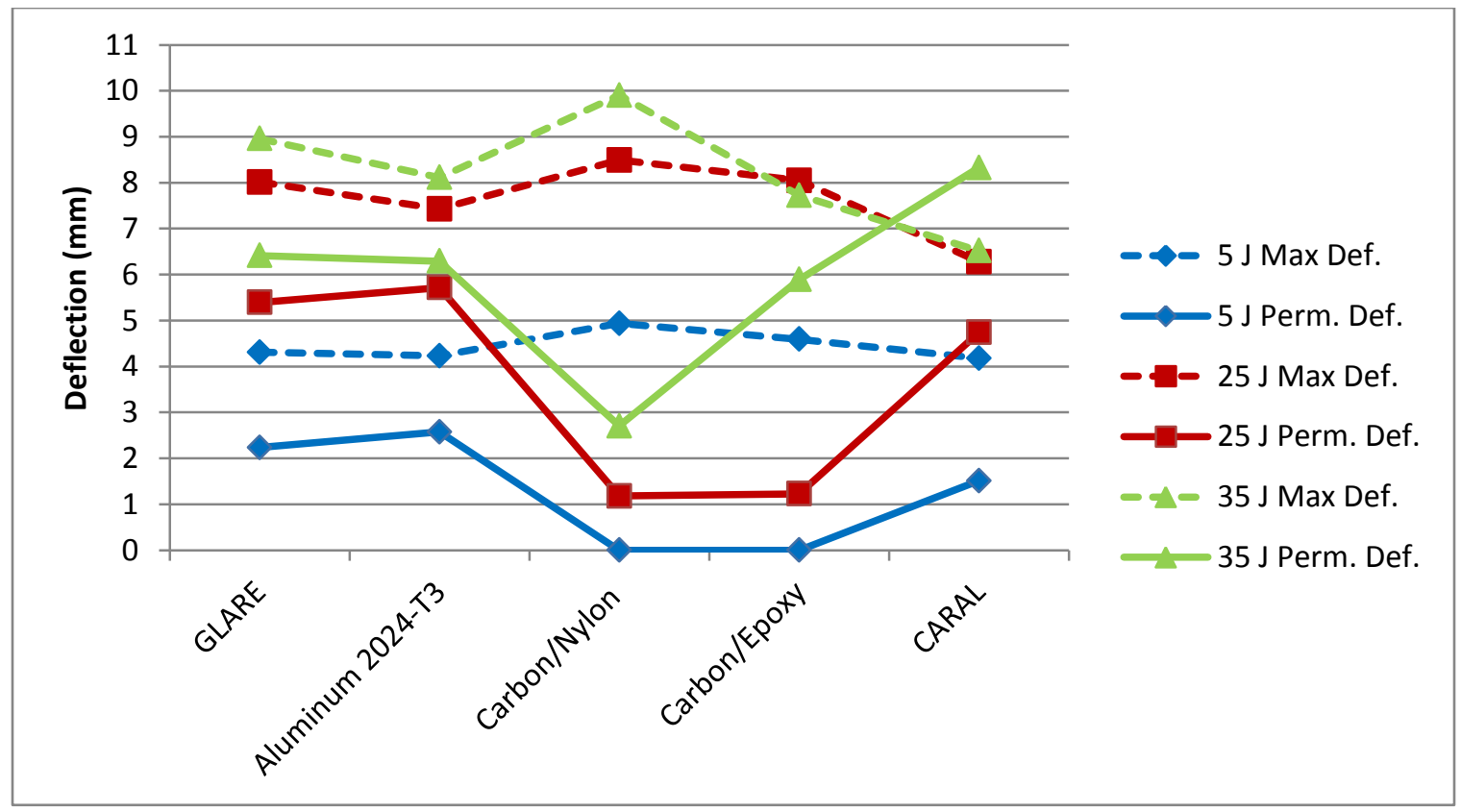

Figure 31: Maximum and permanent deflections at increasing low energy impacts 
A dent is visible to the naked eye at a depth of $1 \mathrm{~mm}$ [74] and so at impact energies as low as $5 \mathrm{~J}$, visible damage can be seen on the impact side of the FMLs and aluminum 2024-T3. GLARE and aluminum 2024-T3 are shown to have similar max deflections and permanent dent depths at every energy level. Carbon composite panels are better at resisting denting as they can reach an impact energy of 25 joules while maintaining a barely visible dent. Identical max deflections at 25 and 35 joule impact energies for carbon/epoxy and CARAL indicate their deflection limit has been reached at $8 \mathrm{~mm}$ and $6.5 \mathrm{~mm}$ respectively. Overall, carbon/nylon has the best dent resistance at low impact energies but also the highest deflections at max load. Upon impact the carbon/nylon panel is therefore most efficient at storing and transferring the energy from and back to the impactor without incurring visible damage on the impact side. In the aluminum 2024-T3 and FML panels a lot of the impact energy is stored as plastic deformation at the site of impact and not transferred back to the impactor.

Although carbon/epoxy and carbon/nylon have barely visible dent depths at an impact energy of 25 joules, from Figure 29 it is shown that at 15 joules visible damage exists in both materials on the non-impact side. First signs of visible damage on the non-impact side (Figure 29) were found to be more severe than that on the impacted side. This is due to the high tensile bending stresses present on the non-impact side where the largest outof-plane deformations are directly beneath the impactor. Visible damage on the nonimpact side of the structure is thus more of a structural integrity concern than an aesthetic one. As an example, the box bed of a pick-up truck is regularly subjected to low-velocity impacts, yet it must also regularly endure heavy static loads. For carbon/epoxy and 
carbon/nylon panels the first visible damage is represented by matrix cracking and fibre breakage in the layer farthest from impact. For FMLs and aluminum 2024-T3 the first sign of visible damage on the non-impacted side is in the form of plastic deformation followed by the development of a crack. Plasticity is difficult to quantify and compare to composite damage modes; thus first signs of cracking in the aluminum will be used for comparison purposes. Measured impact parameters at the first sign of visual damage on the non-impacted side are given in Figure 32.

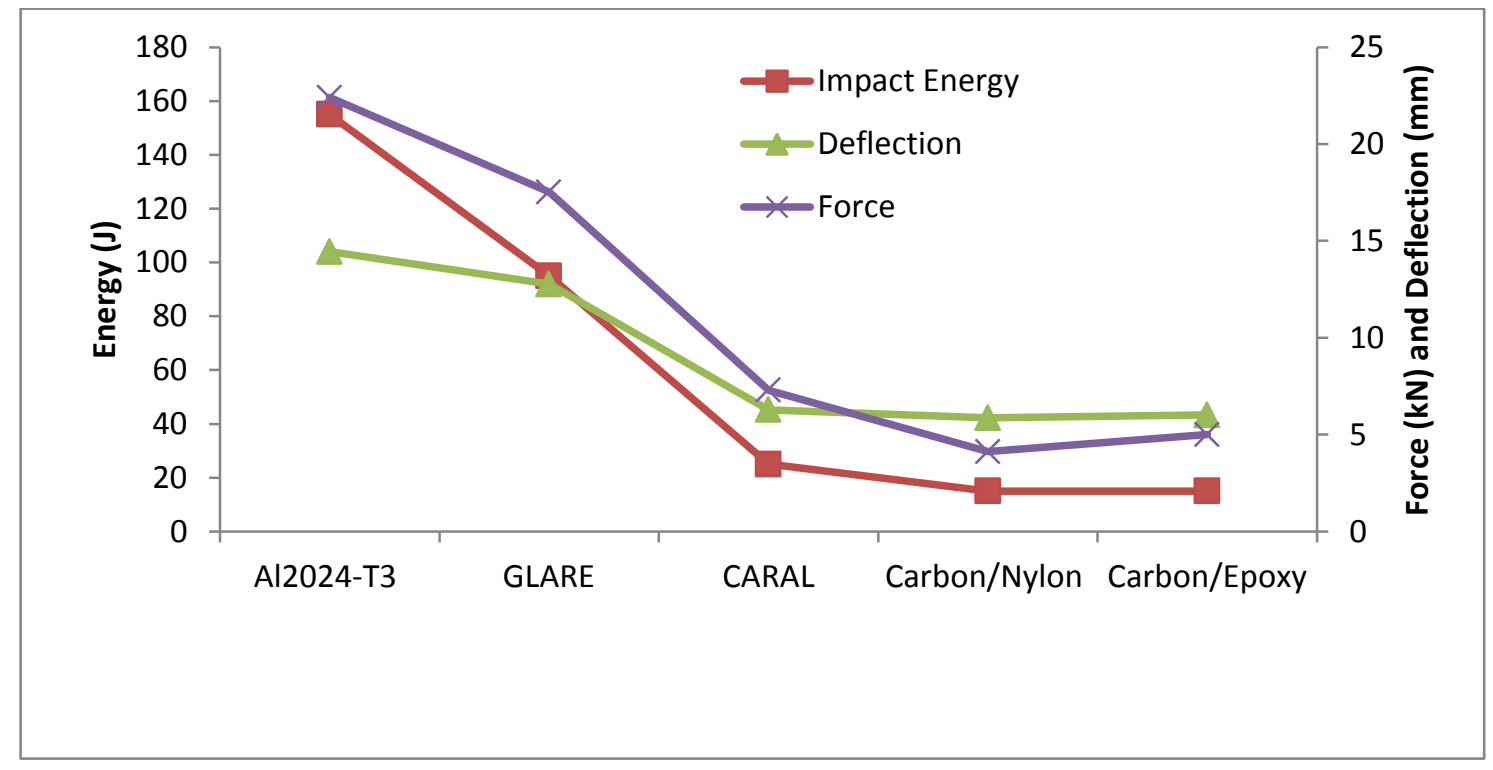

Figure 32: Impact parameters at the first sign of visible damage on the non-impact side

When examining visible damage on the non-impact side it is aluminum 2024-T3 and GLARE that perform best. Considering the material properties in Table 5 it is clear that strain to failure plays an important role in delaying visible damage on the highly strained non-impact side. Aluminum 2024-T3, with the highest strain to failure of $18 \%$, requires the largest force, deflection, and energy to crack. Controlling variables of highest interest 
for the visible damage failure limit are deflection and energy. As demonstrated in Figure 32, GLARE is close to matching the deflection of monolithic aluminum. However, in GLARE the sandwiched layers of S2 glass, with an inferior strain to failure of $4.5 \%$, cause cracking in the outer aluminum layer at an impact energy 65 joules lower than that of aluminum 2024-T3. The visible damage in aluminum 2024-T3 and GLARE panels could be better related if the plasticity regions could be quantified at various deflections and impact energy levels. This would provide a better understanding of how the visible damage propagates in these materials. Panels containing carbon fibres, with the lowest failure strain of approximately $1.6 \%$, are the first to show cracking/fibre breakage as shown in Figure 32. The addition of thin aluminum 2024-T3 layers did not help to increase the visible damage limit of carbon/epoxy in CARAL.

\subsubsection{Break Failure Limit}

The break failure limit represents the start of severe damage during impact at which point the material is no longer able to sustain impactor loads. There is great interest in this failure limit for automotive applications that are required to absorb significant deflection at high impact energies before losing their load carrying capacity. This would apply to any automotive component that comes in contact with a compliant support. An example would be an outer door panel which is required to withstand side impact with a car or pole and transfer the load to a door beam without puncturing. Energy, deflection, and load at the break point in each material are compared in Figure 33. 


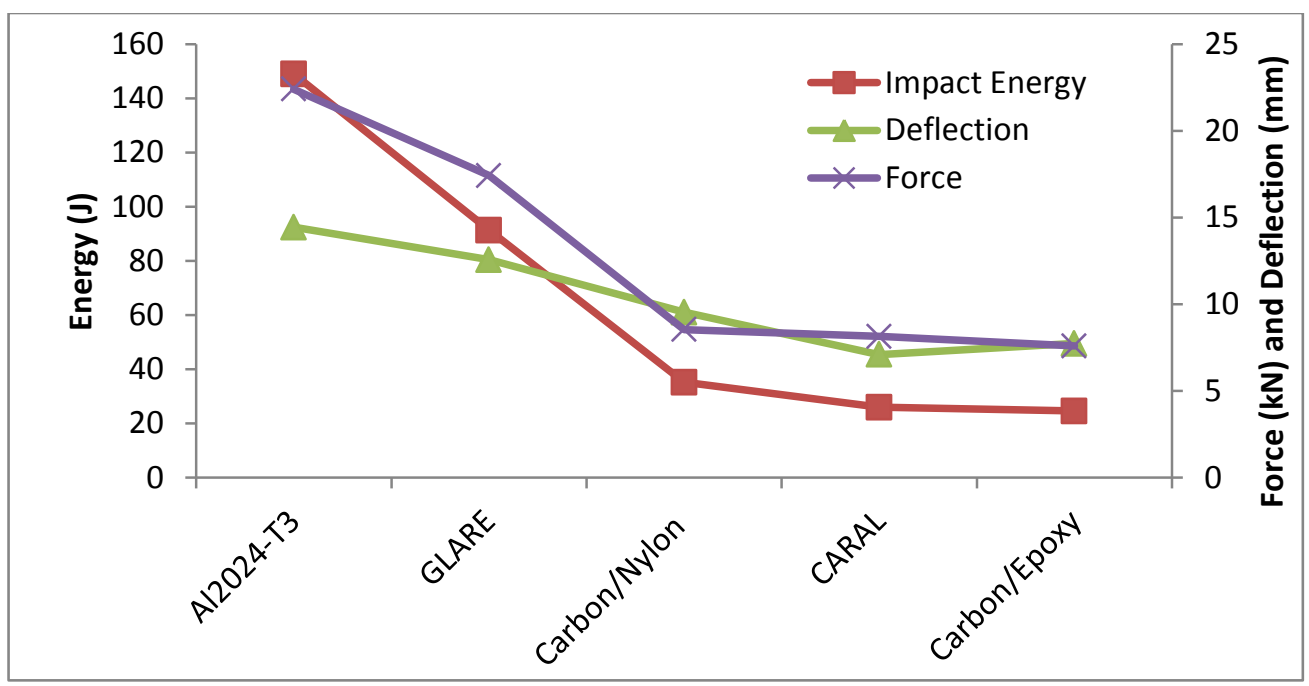

Figure 33: Impact parameters at the break point

It is noticed that the measured impact parameters at the break point are similar in value to those gathered at the first sign of visible damage on the non-impact side in Figure 32. This is expected as visible damage on the non-impact side represented fibre breakage and/or metal cracking after which greater loads cannot be sustained at much higher impact energies. It is then confirmed that visible damage on the non-impact side signifies the start of structural degradation in the panels. Aluminum 2024-T3 is observed to have superior impact parameters at the break failure limit over all other materials tested with GLARE being the closest to match the same energy, force, and deflection. An interesting outcome is the higher break point parameters achieved by carbon/nylon over the CARAL fibre metal laminate. Once again it is realized that the outer aluminum panels in CARAL have offered little improvement over a carbon/epoxy panel of the same thickness at the break point. Just as for the yield failure limit, deflection is the controlling variable of interest in most automotive applications that require high breaking points (Figure 8). The difference in maximum deflection between aluminum 2024-T3 and GLARE is less than 2 
$\mathrm{mm}$ at the break point. A significant constraint in contrasting the deflection from instrumented LVI tests is only the deflection at one point, centre of the panel, can be obtained. In automotive components that come in contact with compliant supports it is imperative that the contact area be maximized for optimal load transfer and energy absorption. A full field profile for the out-of-plane displacement of the panels would therefore be beneficial in comparing the deflection capacity of materials.

\subsubsection{Total Failure Limit}

Few automotive applications specify the total failure limit as the response feature of interest. However, lightweight materials with high energy absorption capabilities are highly sought after in the automotive industry for the manufacturing of crash structures. The energy absorption limits produced from clamped boundary conditions and biaxial membrane deformation caused by impact in this research can be used as a preliminary evaluation step in choosing materials for crash structures that could include side door beams or B-pillars. The energy absorption and puncture characteristics of the lightweight materials in this study are presented in an energy profile diagram in Figure 34. In this research complete puncture is defined for each material when energy absorption equals the impact energy and the impactor does not rebound [30]. Not shown in Figure 34 is the decrease in energy absorption at impact energies beyond the puncture threshold since the impactor retains some of its kinetic energy [24, 70]. 


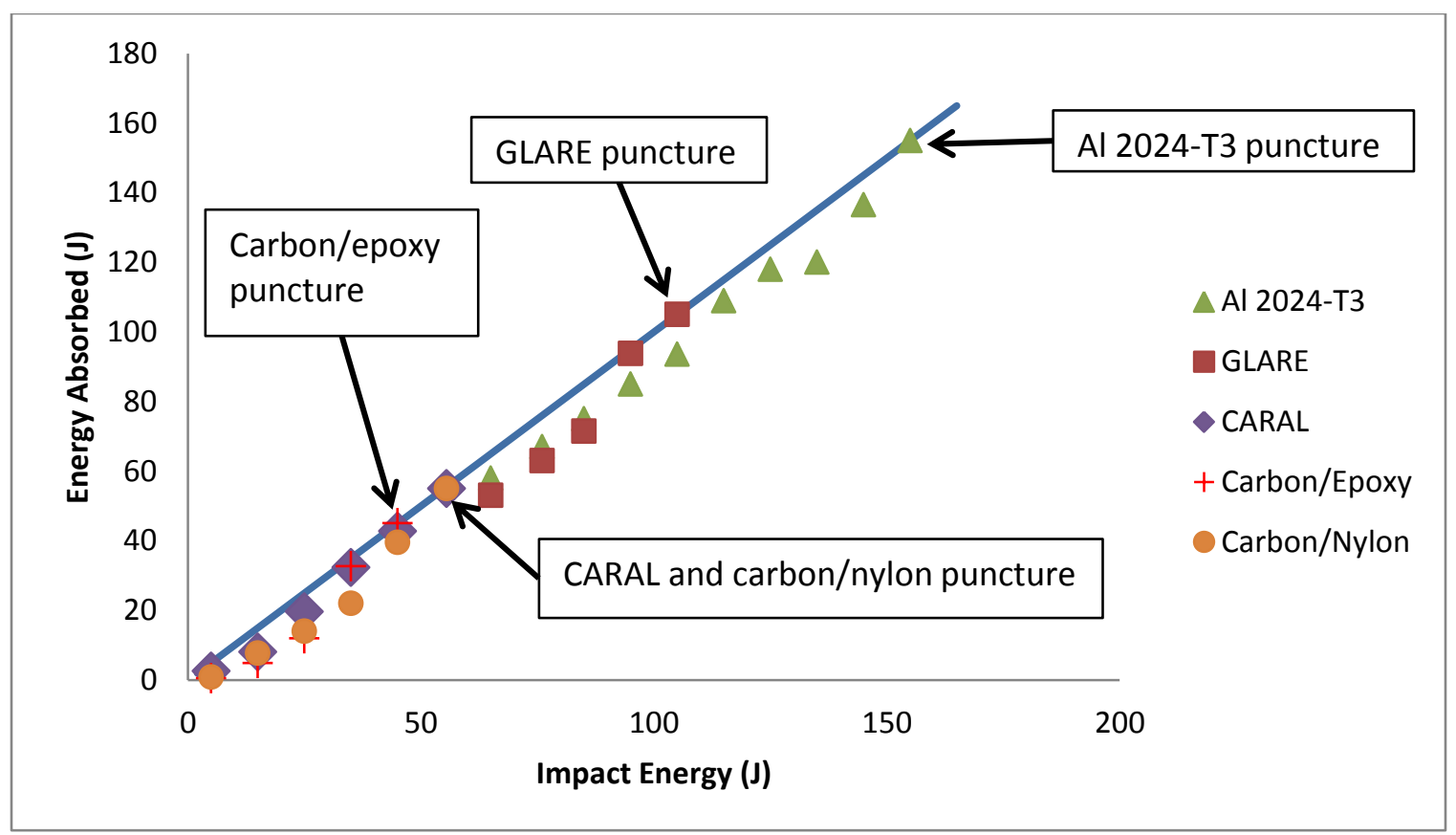

Figure 34: Energy profile diagram emphasizing impact energies to cause puncture

Aluminum 2024-T3 requires the greatest energy to puncture at 155 joules which is 50 joules more than the next material, GLARE at 105 joules. Carbon/epoxy represents the lowest end of the energy absorption spectrum at 45 joules, while CARAL and carbon/nylon both puncture at 55 joules. Energy absorption limits and resistance to puncture can be explained by comparing the stress-strain curves in Figure 35 for the laminates and their material constituents. The curves are gathered from literature where stacking sequences and FML configurations are slightly different than those used in this study. 


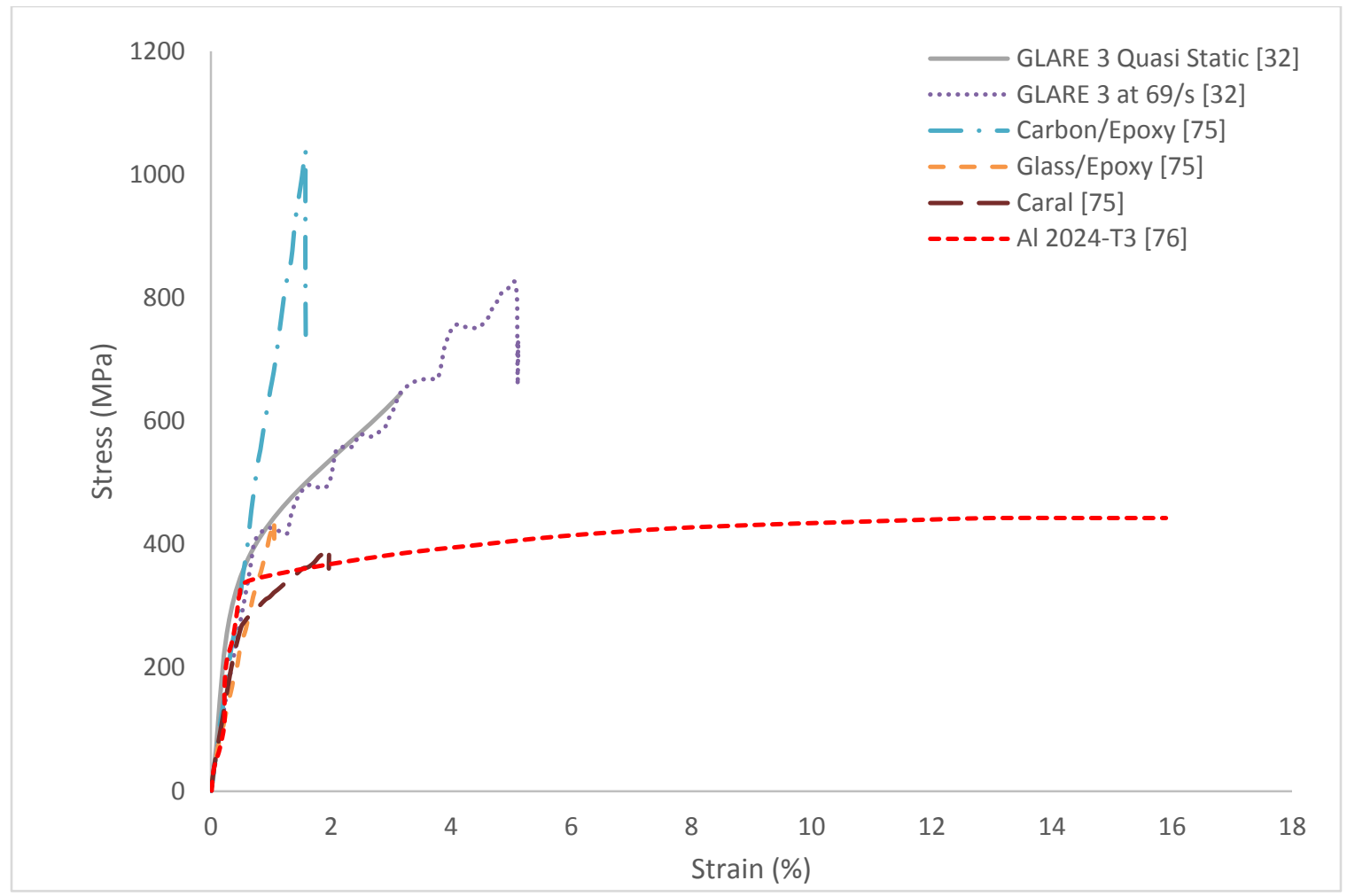

Figure 35: Stress-strain curves of the various laminates and their constituents

Carbon/epoxy has superior stiffness to monolithic aluminum 2024-T3 (Figure 35), yet it punctures at a lower impact energy (Figure 34). This is a result of differences in strain energy densities or area under the stress-strain curves. Monolithic aluminum 2024-T3's large plastic deformation region and high failure strain give it the best strain energy density among all materials tested. This correlates well with its superior energy absorption and puncture limits in the LVI impact tests. GLARE and CARAL's inner composite cores are shown to gain strain energy density with the addition of aluminum layers as they develop a plastic deformation region and higher failure strains (Figure 35). Again observations in the stress-strain curve run parallel with the total response in LVI tests since the addition of aluminum to carbon/epoxy in CARAL increased the puncture 
energy by 10 joules. GLARE's energy absorption and puncture limit dominance over the other laminates is of course related to the strain rate dependent mechanical properties of glass-fibres that can increase the strain energy density at higher strain rates (Figure 35). Carbon/nylon is estimated to have a similar stress-strain curve to that of CARAL since they puncture at the same impact energy. Improved toughness with a thermoplastic matrix is then beneficial for raising strain energy density analogous to that achieved by adding the aluminum layers to the thermoset matrix in a CARAL panel.

An approximate panel thickness of $1.2 \mathrm{~mm}$ was selected in this study to represent automotive body panels. It has been shown that under constant thickness aluminum 2024$\mathrm{T} 3$ provides the highest energy absorption before puncture; however it is also the heaviest panel tested. Specific energy absorptions of each material are contrasted in Figure 36 where it is evident that aluminum 2024-T3 is the most efficient energy absorbing material based on energy absorption per mass. Energy absorption has been normalized based on density since it takes account of the differences in mass and slight variations in panel thicknesses. 


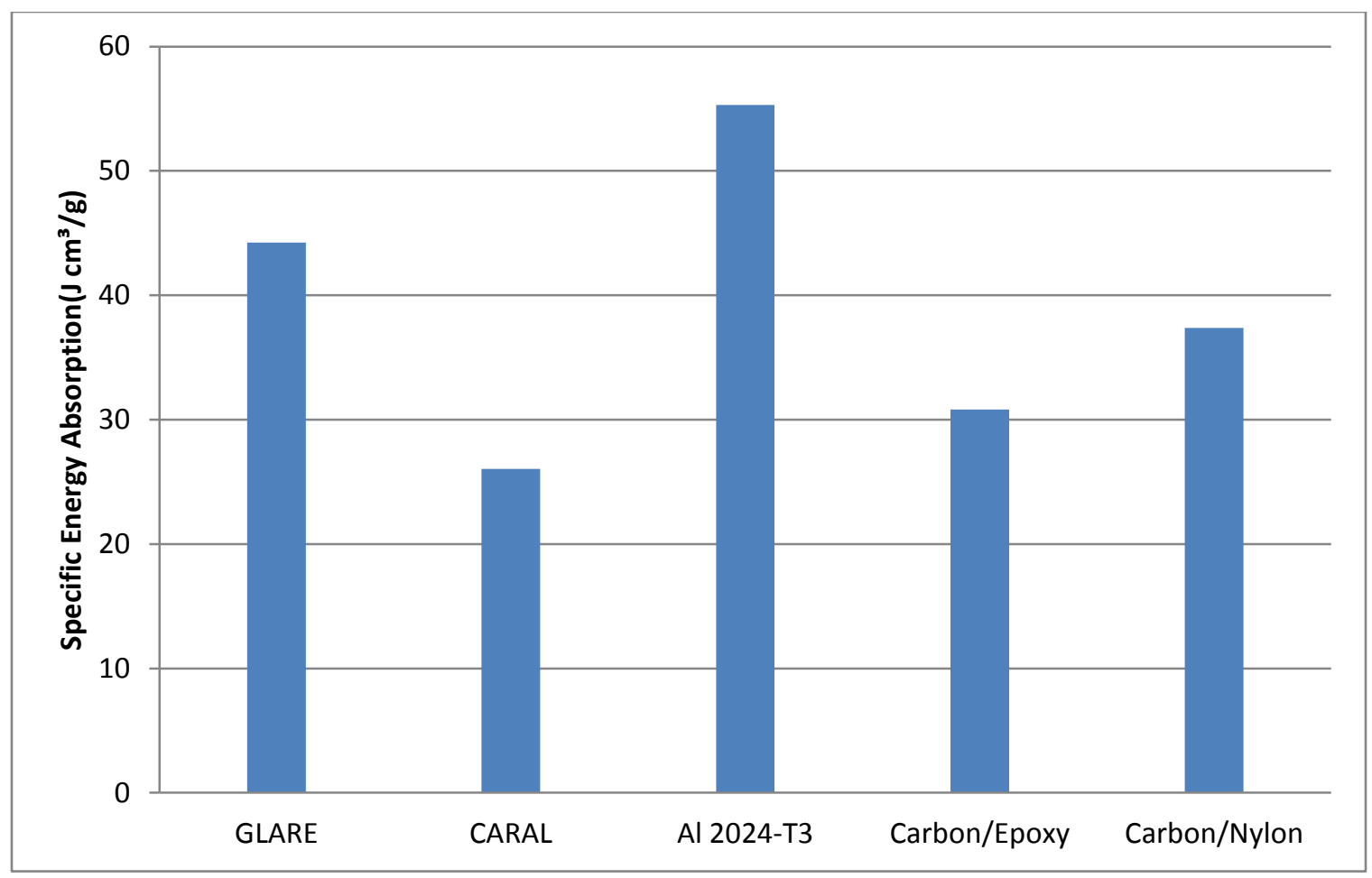

Figure 36: Specific energy absorption calculated at puncture

To match the energy absorption of aluminum the thickness of all other lightweight materials would have to be increased to such an extent that their mass would exceed that of the aluminum. This conclusion has its limitations however as the specific energy absorption of FMLs was found to increase with thickness in reference [64]. Also, the tailorability of composites and FMLs leaves the possibility that different layup orientations or metal-to-composite layer configurations could provide improved specific energy absorption. For instance, Moriniere et al. [77] administered LVI impact tests on $1.3 \mathrm{~mm}$ thick aluminum 2024-T3 and GLARE 5 2/1-0.4 panels and concluded GLARE had a specific energy absorption $72 \%$ greater than the aluminum panels. Reasons for discrepancies among Moriniere [77] and the results in this study could be due to the increased thickness of aluminum layers used in GLARE. The more likely reason is the 
differences in boundary conditions and shape of impactor head employed. More specifically, rectangular versus circular unsupported areas and conical shaped as opposed to hemispherical impactor heads. Therefore when considering materials for impact resistance the dependence of impact response on clamping boundary conditions, impactor geometry, and other impact test variables must be appreciated. Future studies should thus strive to simulate the impact conditions that occur in the application of interest. The clamping fixture used in this study has an unsupported region smaller than what may be found in some automotive applications such as an outer door panel or vehicle fender. In these cases a larger unsupported region means greater membrane deformation and more energy absorption especially in GLARE and aluminum 2024-T3.

\subsection{Quasi-Static Indentation Tests}

The purpose of the quasi-static indentation tests is to simulate a dynamic impact event while allowing a DIC stereovision setup to capture full field displacement and strain measurements. The full field DIC data offers many advantages over the instrumented drop weight test data in evaluating potential lightweight automotive materials. These advantages and analysis of the DIC results will be given in this section, but first the limitations of using QSI tests to simulate low-velocity impacts are explored.

\subsubsection{Comparing Quasi-Static Indentation to Low-Velocity Impacts}

The impactor-plate mass ratios for the low-velocity impact tests in this study were greater than 200 which far exceed the ratio of 2 needed to classify an impact response as quasistatic. The equivalency of LVI and quasi-static impact response with full plate 
deformation can be evaluated by comparing the contact force and deflection histories.

Contact force and deflection histories for several materials at various low-velocity impact energy levels are given in Figure 37 to show how the phase of the two impact parameters compares.

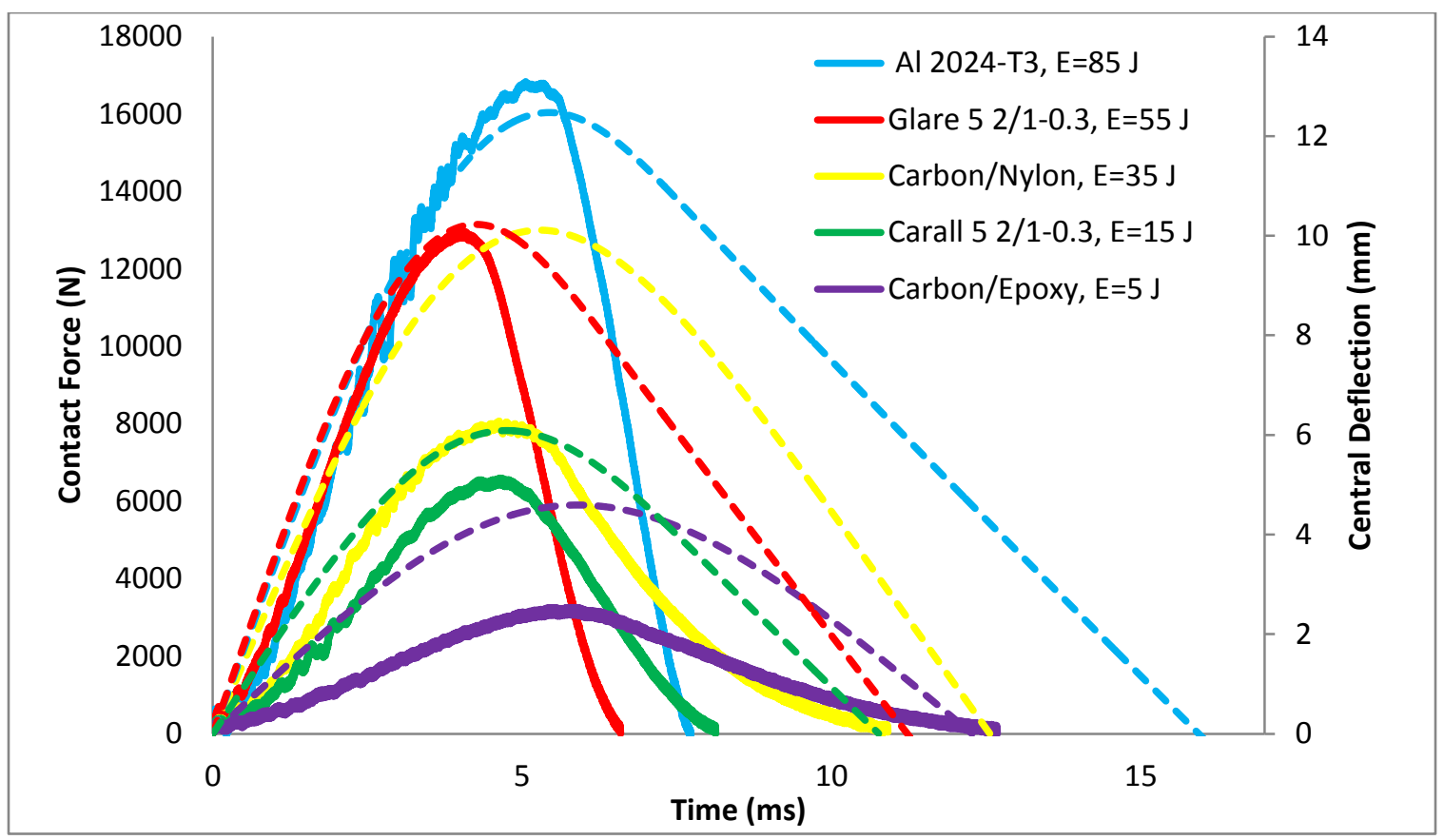

Figure 37: LVI test contact forces (solid line) and central deflections (dotted line) as a function of time

The contact force and deflection histories can be specified as in phase meaning the LVI tests in this study provoke a quasi-static impact response versus the possibility of initiating dynamic wave controlled behaviour. The next step is to compare forces, displacements and energy results between the two tests for each material. This is shown in Figure 38 where the LVI and QSI results are given at a point when puncture occurs. Parameters at puncture were chosen to make first contrasts since it is an easy damage 
state to identify that represents the impact/indent limits of a material. Aluminum 2024-T3 is excluded from these figures because its properties exceeded the $20 \mathrm{kN}$ abilities of the static bench before puncture took place.

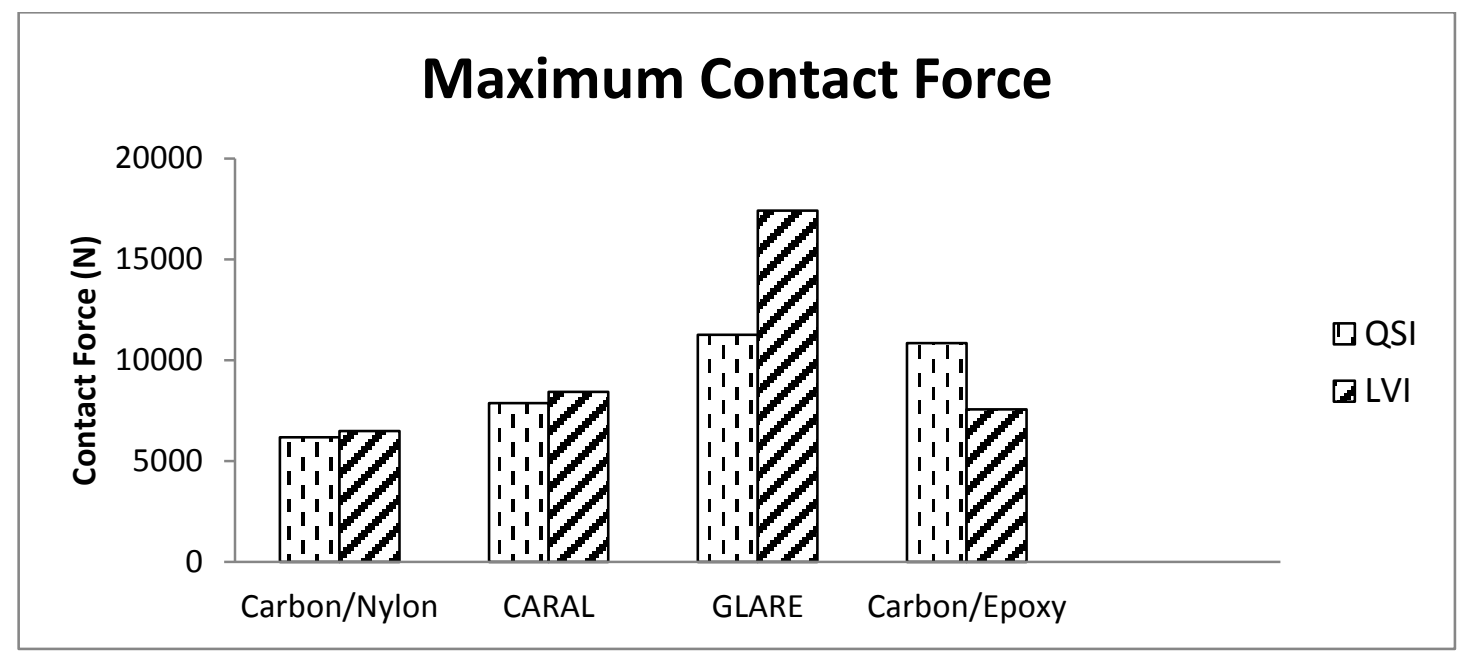

a.)

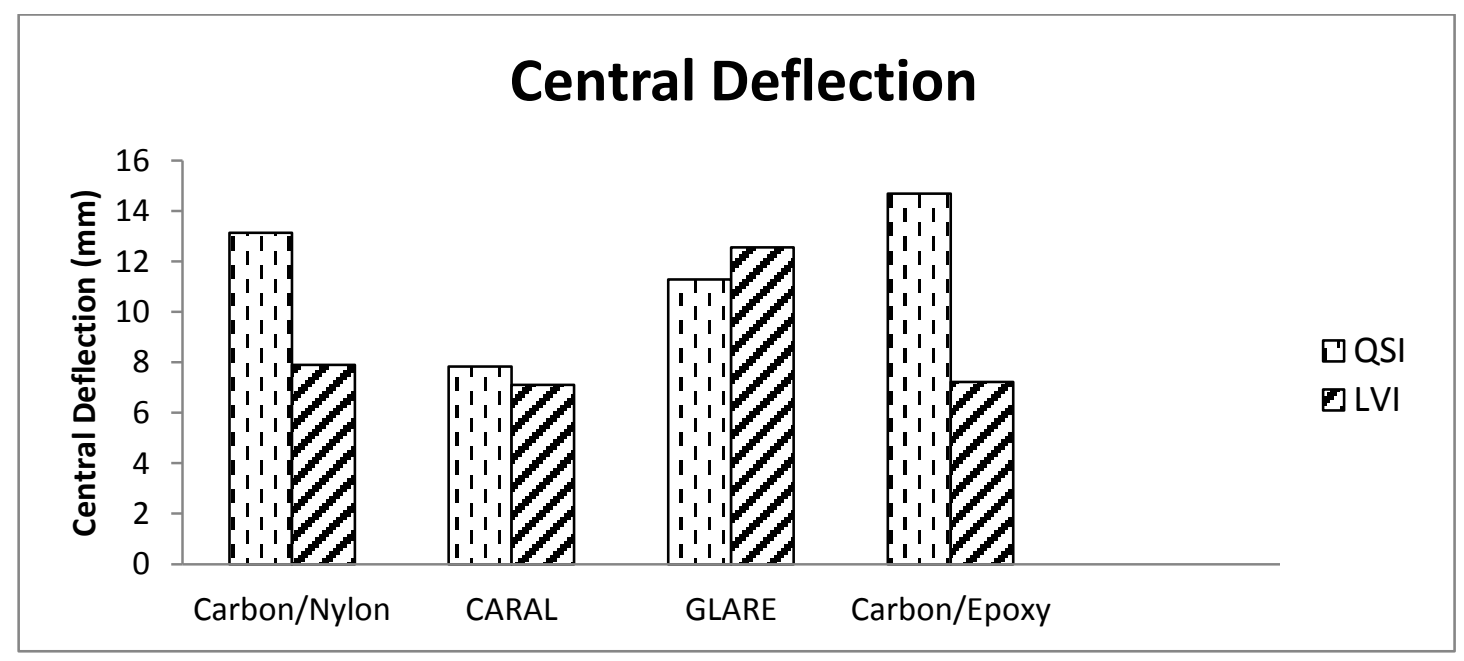

b.) 


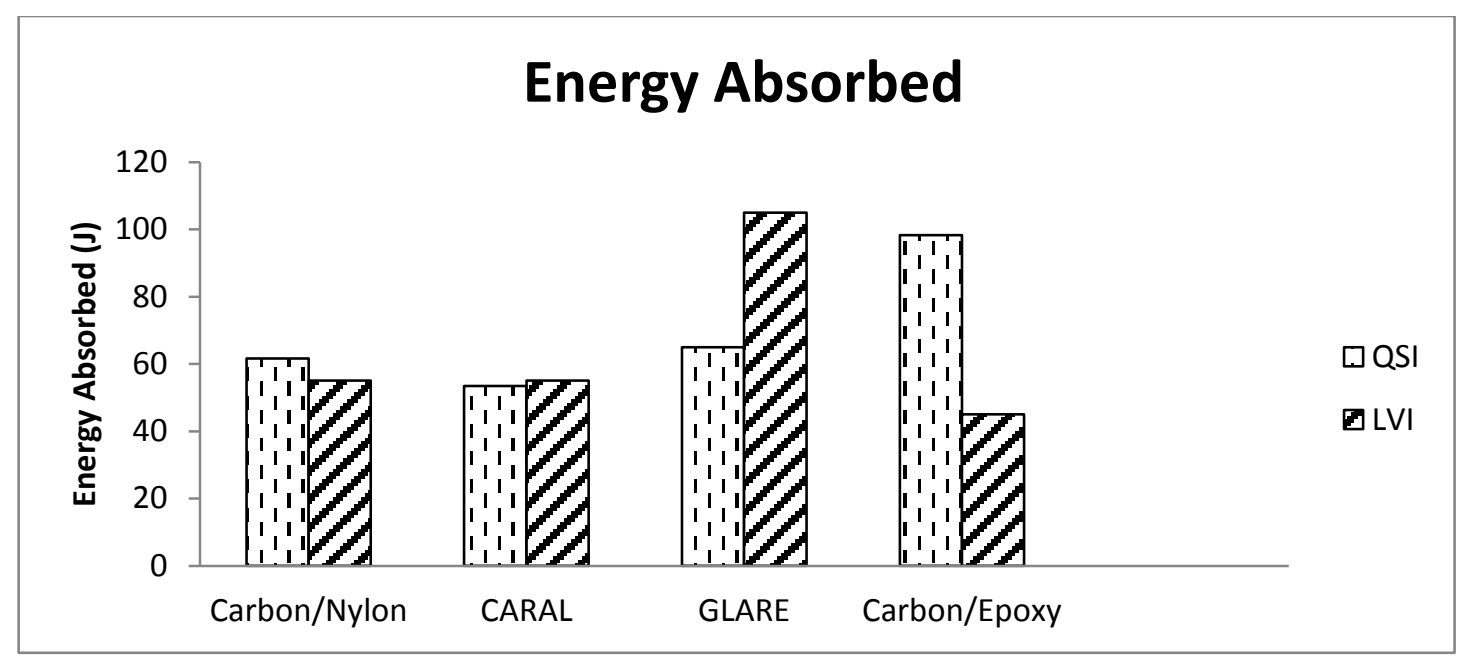

c.)

Figure 38: Contrasting a.) maximum contact force b.) Central deflection at maximum contact force and c.) energy absorbed between LVI and QSI tests at puncture

The ability of QSI tests to simulate low-velocity impacts resulting in puncture is shown to be dependent on the type of material and impact parameter that is being contrasted. Carbon/epoxy's low-velocity impact resistance is not well simulated by QSI tests which provides an over estimation of maximum contact force, central deflection, and energy absorbed. The opposite is true for CARAL as all impact parameters at puncture match well between the two tests. For carbon/nylon contact force and energy absorbed are similar but central deflection in QSI tests far exceeds that found in LVI. GLARE is unique in that although the central deflection is similar, in general the LVI results are more favourable to those found during QSI testing at puncture. In LVI tests of GLARE contact force is $35 \%$ greater, central deflection is $10 \%$ higher, and energy absorbed at puncture is $38 \%$ above that experienced in QSI tests. 
Differences in QSI and LVI results at puncture can be linked to specific material properties and characteristics. Carbon/epoxy is known to be very brittle and under dynamic impact loads requires little energy for fibre breakage and matrix cracking to occur at the impact site [32]. This brittle behaviour is less significant in QSI testing as the loading rate is slow enough to allow the high stiffness carbon fibres at the indentation site to evenly re-distribute the load into plate bending without fracture. Figure 39 demonstrates the significant amount of plate bending and membrane deformation during QSI puncture and lack of it during puncture in the LVI test even though indentor and impactor heads of the same dimension were used in QSI and LVI tests respectively.

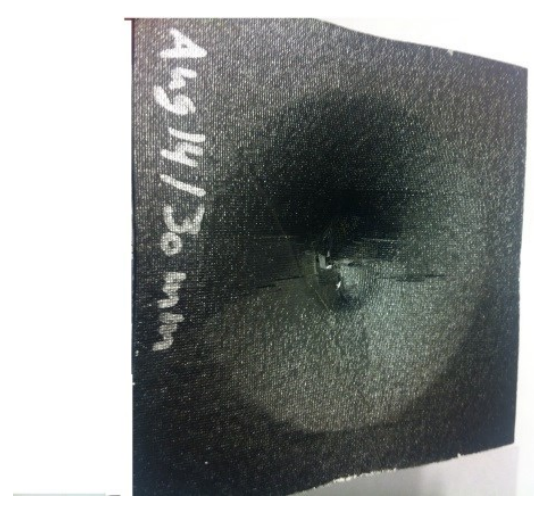

a.)

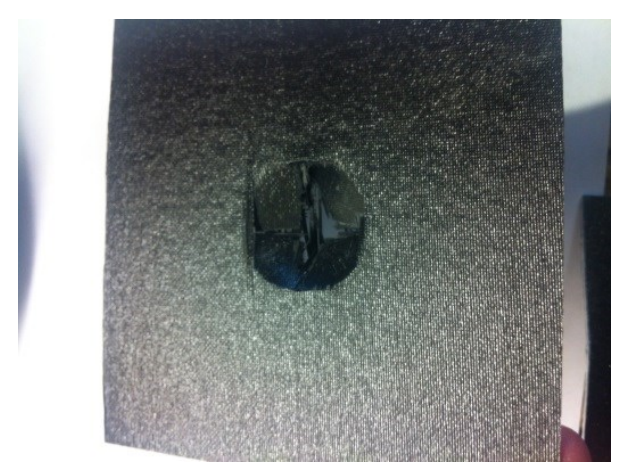

b.)

Figure 39: Damage states at puncture in carbon/epoxy for a.) QSI test showing significant plate bending and membrane deformation and b.) LVI test showing brittle fracture

The thermoplastic matrix in carbon/nylon is tougher (less brittle) than the thermoset matrix in carbon/epoxy resulting in a closer comparability between the two tests. Damage 
states at puncture are still very different for carbon/nylon as plate bending and membrane deformation are present in QSI tested samples but not in LVI (Figure 40).

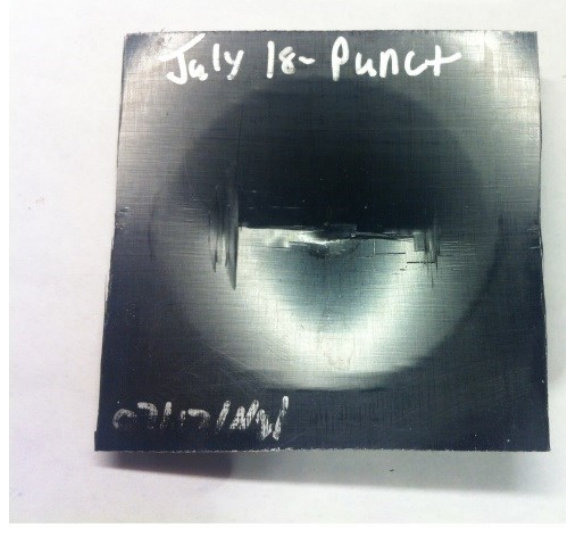

a.)

Figure 40: Damage states at puncture in carbon/nylon for a.) QSI test showing significant plate bending and membrane deformation and b.) LVI test showing brittle fracture

In the case of CARAL the outer aluminum 2024-T3 layers compensate for the brittle behaviour of the inner carbon/epoxy layers through their ability to deform plastically. This balance of brittleness and toughness in CARAL has produced the closest matching of puncture parameters gathered from QSI and LVI testing. Damage states at puncture in CARAL are also identical amidst the two tests as shown in Figure 41. Aluminum cracking in CARAL first appears on the non-impact side and runs parallel to the $0^{\circ}$ fibre direction. 

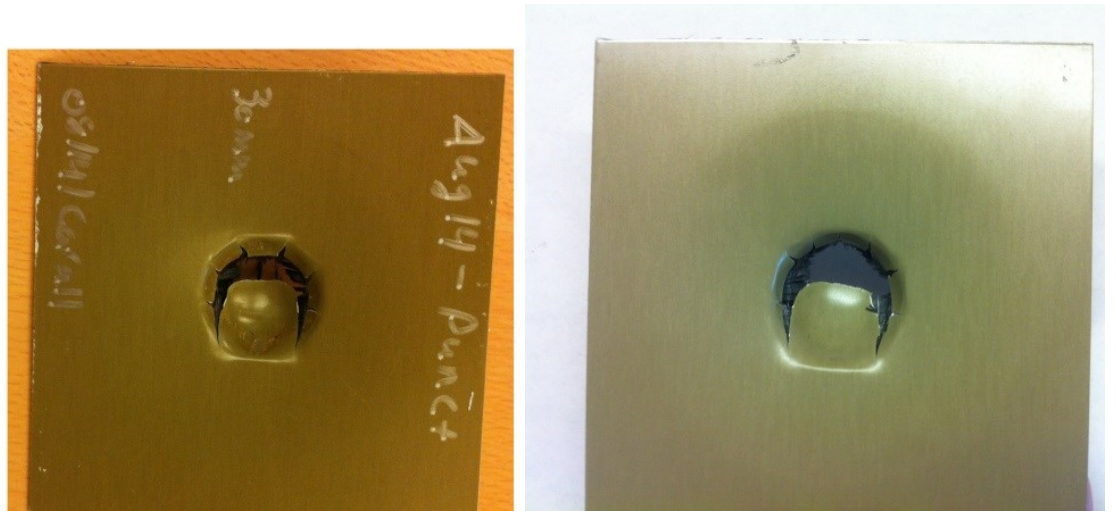

a.)

b.)

Figure 41: Damage states at puncture on the impact side in CARAL for a.) QSI test b.)

\section{LVI test}

GLARE's ability to perform better in LVI testing is a direct result of the increase in strength and failure strain at high strain rates for glass fibres. Impactor velocity in an LVI test will dictate the strain rates within an impacted sample as demonstrated in Figure 42 for the finite element low-velocity impact model of aluminum 2024-T3 (more FEA results will be presented in section 6.2.2.3). Energies and velocities in Figure 42 coincide with those used in the LVI tests. 


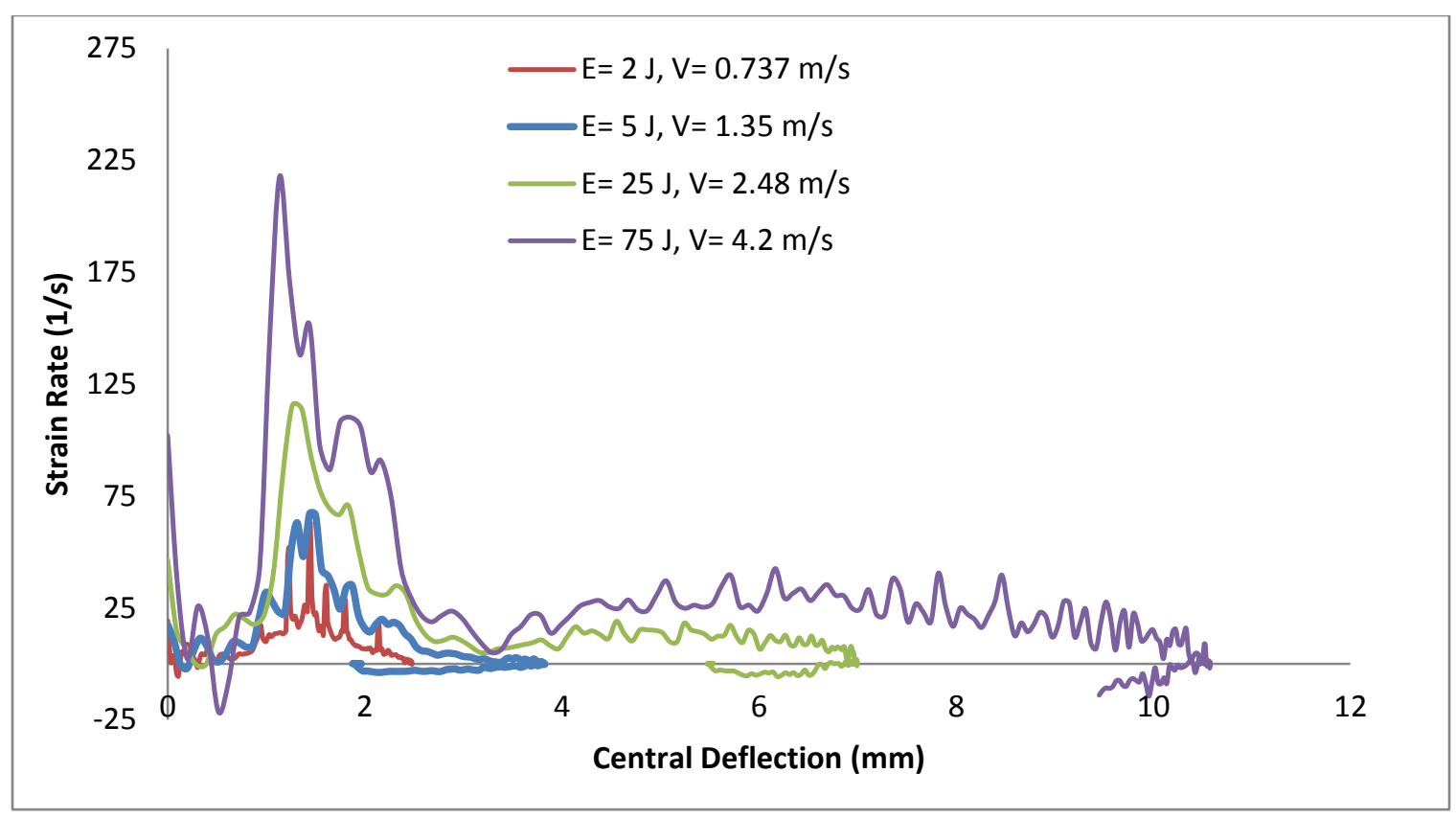

Figure 42: Dependency of strain rate on impactor velocity in FEA low-velocity impact model of aluminum 2024-T3

Peaks of high strain rate occur shortly after the impactor makes contact with the plate specimen and as expected at lower impactor velocities the strain rates are also lower. As impactor velocity and impact energy increase there is sustained high strain rates through the full deflection of the plate. Assuming GLARE exhibits similar strain rate response as aluminum 2024-T3 (see similarities in force-deflection curves in Figure 53), it is the strain rates that are sustained for a longer period of deflection that have the greatest effect on the mechanical properties of the glass-fibres. Noteworthy changes in strength and strain to failure in glass-fibres were found to take place at strain rates above $10 \mathrm{~S}^{-1}$ [78]. The strain rate sensitivity of aluminum 2024-T3 is also found to initiate above a strain rate of $10 S^{-1}$ [72], although property changes are not as great as in the glass-fibres [26]. Hence, the QSI and LVI resistance parameters may correlate better at lower impactor 
velocities and impact energies for GLARE and aluminum 2024-T3 as strain rate effects are minimized. Force-deflection curves plotted in Figure 43 for GLARE show that at a lower impactor velocity of $2.5 \mathrm{~m} / \mathrm{s}$ and at the same impact/indent energy of $25 \mathrm{~J}$ the maximum contact force is identical for the two tests. The deflection however is still slightly lower in the LVI test as it was at puncture (Figure $38 \mathrm{~b}$.)). At a higher impactor velocity of $3.8 \mathrm{~m} / \mathrm{s}$ the strain rate strengthening effects of GLARE are dominant as a higher contact force is reached for the same impact/indent energy level of $65 \mathrm{~J}$ without puncturing in the LVI sample. In maintaining the same impactor velocity of $3.8 \mathrm{~m} / \mathrm{s}$, but by adding more mass to increase impact energy, the increased strength of GLARE is able to absorb more energy until puncture in the LVI test. This is directly related to the increased area below the stress strain curve for GLARE at high strain rates as the failure point is extended (Figure 35).

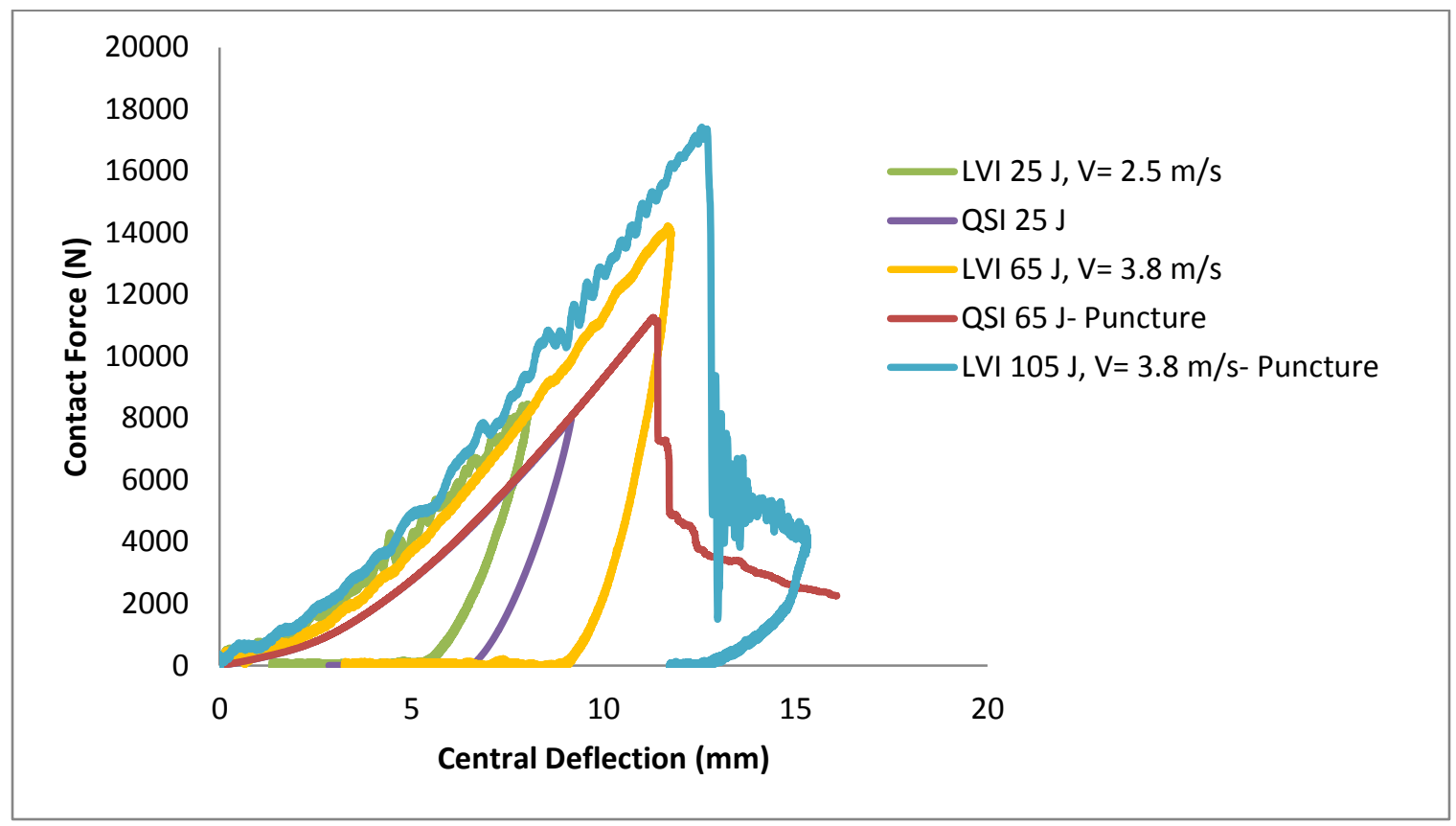

Figure 43: Comparing force-deflection curves for QSI and LVI tests on GLARE 
The effects of strain rate strengthening are examined more closely in Figure 44 for both GLARE and aluminum 2024-T3 by contrasting QSI and LVI contact force and deflection behaviour at increasing LVI impactor velocities. Each data point in Figure 44 is contrasted at identical indent/impact energy levels where only energy levels below QSI puncture are considered. For GLARE force and deflection parameters are indistinguishable among the quasi-static and low-velocity impact tests at a velocity of $0.74 \mathrm{~m} / \mathrm{s}$. As velocities increase to between $1.4 \mathrm{~m} / \mathrm{s}$ and $2.8 \mathrm{~m} / \mathrm{s}$, strain rate effects are minimal but present since contact force is greater and deflection lower in the LVI test. Therefore, it can be concluded that QSI and LVI tests invoke similar responses in GLARE for impactor velocities below approximately $3 \mathrm{~m} / \mathrm{s}$ as strain rate effects are minimized. Aluminum 2024-T3 has shown to provide better comparability between QSI and LVI test parameters as the contact forces are identical and LVI deflections on average slightly lower through an impactor velocity range of $0.74 \mathrm{~m} / \mathrm{s}$ to $4.2 \mathrm{~m} / \mathrm{s}$. Thus strain rate strengthening effects in aluminum 2024-T3 are minimal compared to GLARE.

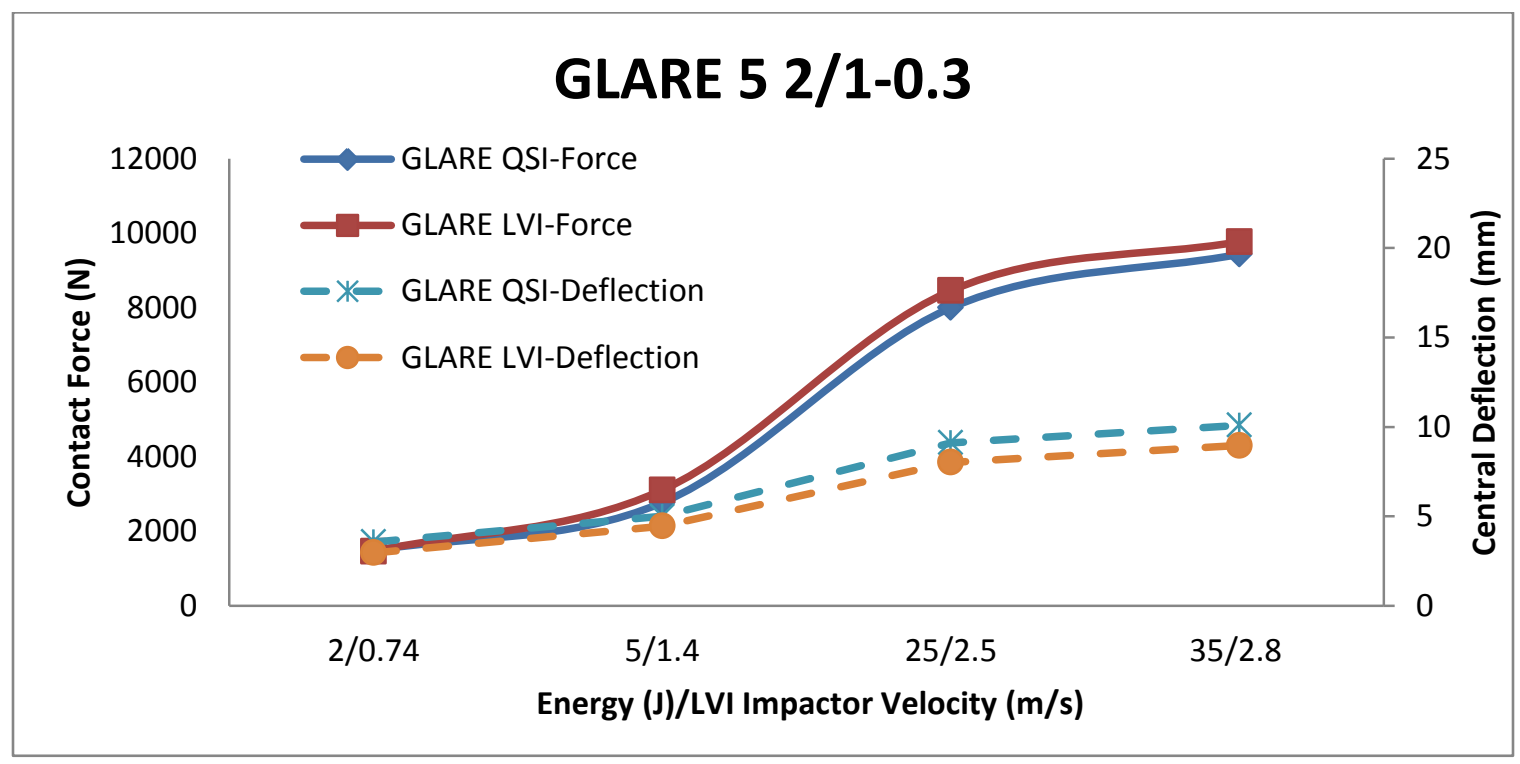

a.) 


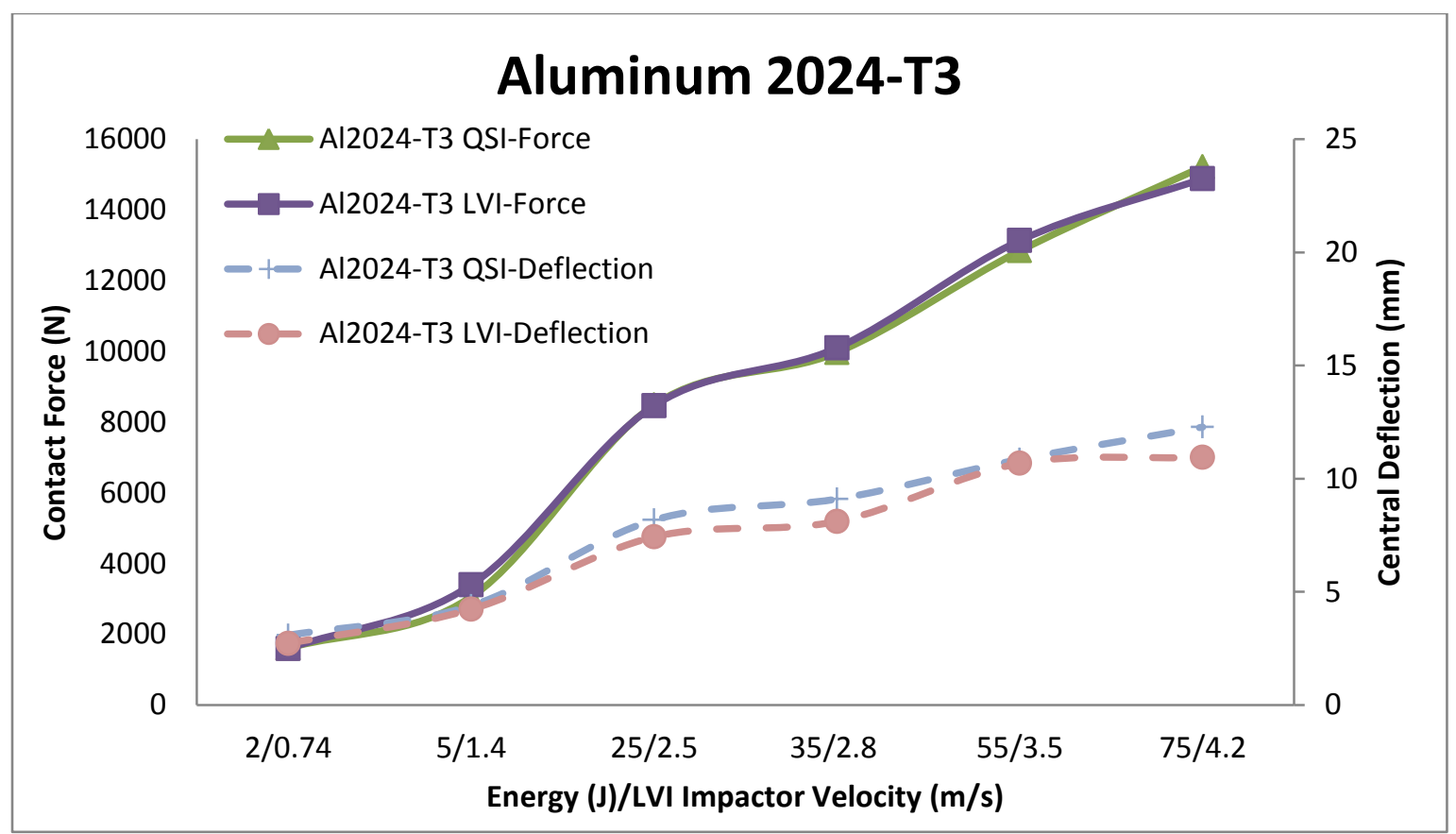

b.)

Figure 44: LVI and QSI parameter comparability for increasing LVI impactor velocity in

a.) GLARE and b.) aluminum 2024-T3

By chemically etching the GLARE samples in a hydrochloric acid solution the outer aluminum layers have been removed in Figure 45 to fully expose damage states at puncture in LVI and QSI tests. 


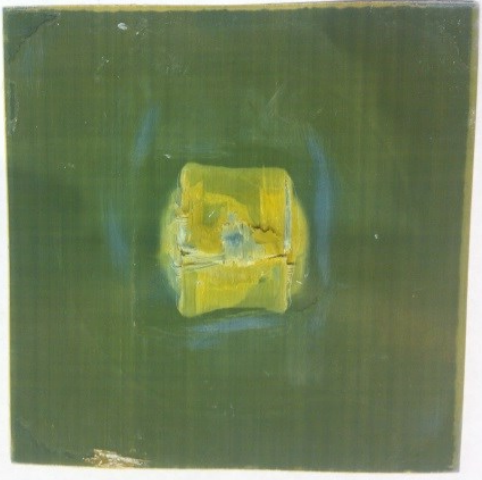

a.)

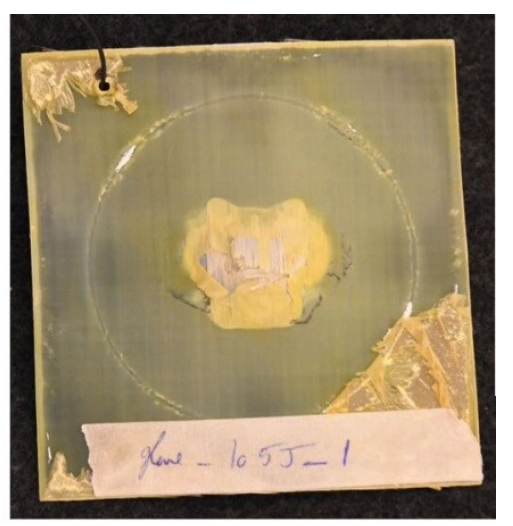

b.)

Figure 45: Chemically etched samples of a.) GLARE QSI specimen and b.) GLARE LVI specimen at puncture

Strain rate strengthening effects in GLARE during low-velocity impact do not appear to alter the damage state at puncture when compared to a quasi-statically loaded sample. In both tests the damage is composed of fibre breakage and delamination restricted to the site of impact. Aluminum cracking in GLARE first appears on the non-impact side and runs perpendicular to the $0^{\circ}$ fibre direction. Damage states at impact/indent energies below that to cause puncture are presented in Figure 46 for both CARAL and GLARE. 


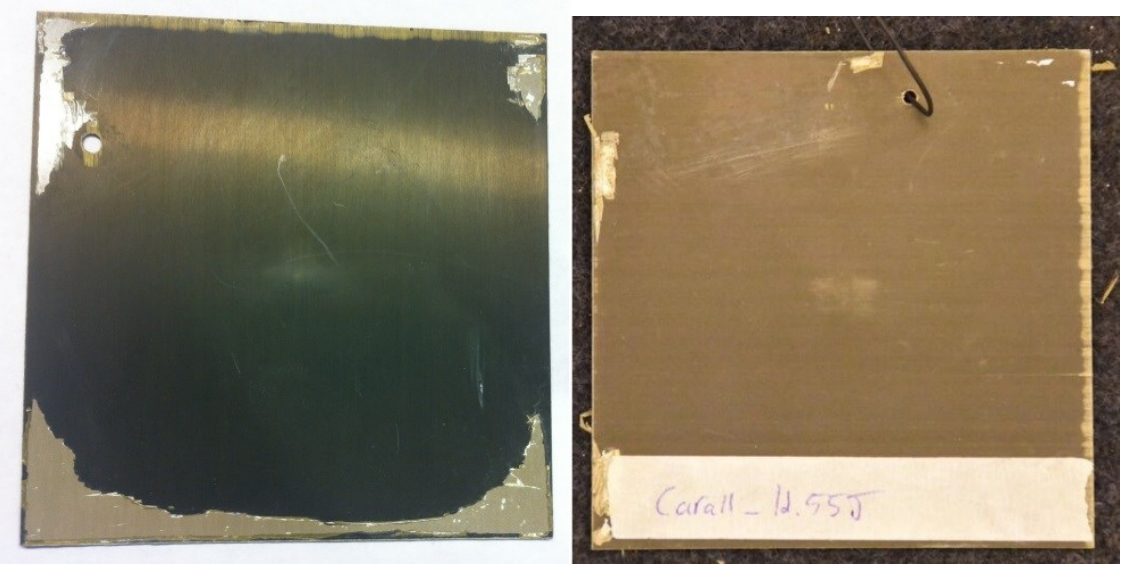

a.)

b.)
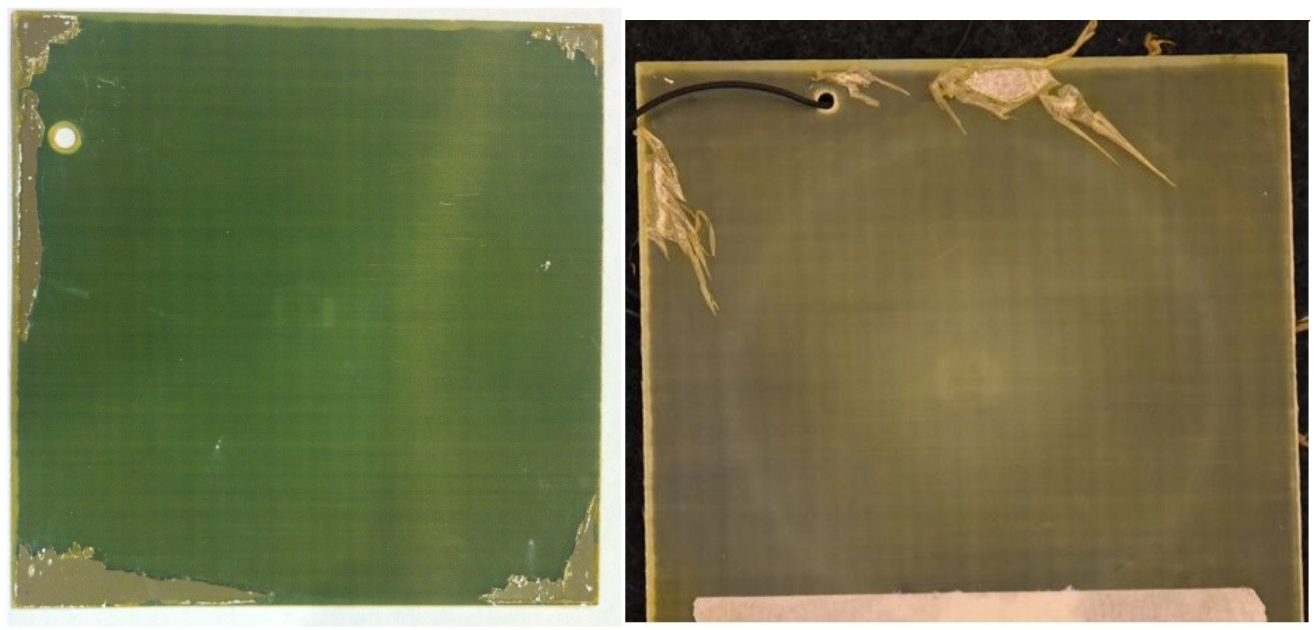

c.)

d.)

Figure 46: Chemically etched samples of CARAL at an impact/indent energy of $12.55 \mathrm{~J}$ for a.) QSI and b.) LVI and GLARE at an impact/indent energy of $25 \mathrm{~J}$ for c.) QSI and d.) LVI

Even at lower impact energies the damage states are identical between low-velocity and quasi-static test samples where delamination and fibre breakage does not occur. 


\subsubsection{Advantages of Using DIC in Quasi-static Indentation Tests}

Present and prospective automotive materials have been rated for impact resistance based on the parameters gathered from instrumented low-velocity impact equipment. While maximum contact force and energy absorption data has been used to justify material choices, there has been a lack of experimental evidence characterizing the full field deformation behavior of materials under impact loads. This sub-section will explore the advantages of using full field digital image correlation data in QSI tests when considering the impact performance of future automotive lightweight materials. DIC results and analysis will focus on the QSI tests of aluminum 2024-T3 and GLARE since these materials had the best response to low-velocity impacts. Also, quasi-static loading conditions were unable to replicate impact parameters achieved in LVI tests for carbon fibre composite panels. Moreover, the DIC results presented are only applicable for low strain rate induced impact events on GLARE.

\subsubsection{Out-of-Plane Displacement Profiles}

An important safety feature of certain automotive components is the ability to deform sufficiently and engage compliant supports that absorb the majority of impact energy. As mentioned before, an instrumented LVI test only provides deflection at the point of impact. However, global deformation outside the point of impact is of interest for components that must deflect and engage secondary supports. In using DIC during QSI testing the out-of-plane displacement fields (Figure 47 a.) can be obtained for GLARE and monolithic aluminum 2024-T3. To compare the deflection in each material displacement data has been extracted from the DIC fringe plots as shown in Figure 47 a.) to construct the out-of-plane displacement profiles in Figure 47 b.) at increasing 
indentation times. Since the QSI tests are displacement controlled ( $2 \mathrm{~mm} / \mathrm{min})$, the profile peaks are identical at the center of the panel at each indentation time in Figure 47 b.). This is a testament to the repeated accuracy of the DIC setup and the methods developed in chapter 5. By contrasting out-of-plane displacement profiles at identical central deflections differences in displacement outside the contact area are highlighted. At small central deflections the profiles of GLARE and aluminum 2024-T3 in Figure 47 b.) are identical. As central deflections increase to $10 \mathrm{~mm}$ the deflection of GLARE outside the contact zone is greater than that of aluminum 2024-T3. This supports the analytical model findings in [77] where it was found that GLARE absorbs more impact energy in membrane deformation (77\%) than aluminum 2024-T3 (53\%). Although the largest difference in deflections outside the contact zone was just over $1 \mathrm{~mm}$, it is important to note that the unsupported region in both the LVI and QSI tests was $80 \mathrm{~mm}$ in diameter. Also, the indenter head radius was only $15 \mathrm{~mm}$. The unsupported region in a car fender or outer door panel will be much greater, hence the differences in membrane deformation for aluminum 2024-T3 and GLARE will be magnified. Lotus Engineering Inc. [3] determined that the intrusion of door panels during side impact and side pole impacts reached $65 \mathrm{~mm}$ and $160 \mathrm{~mm}$ respectively. 


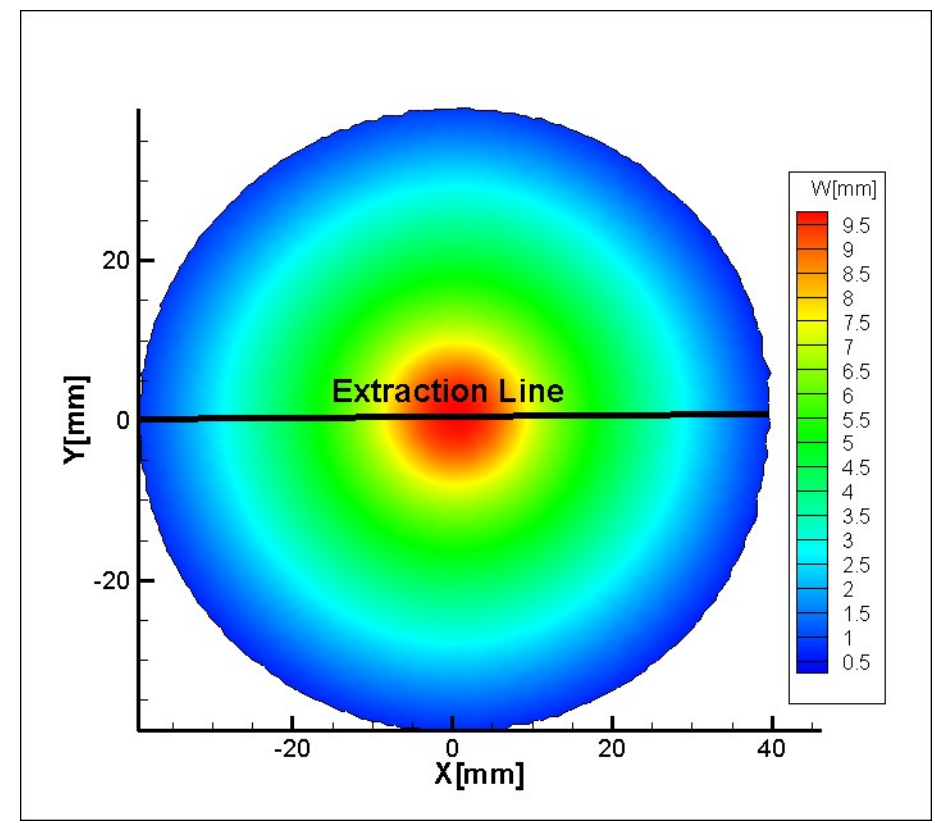

a.)

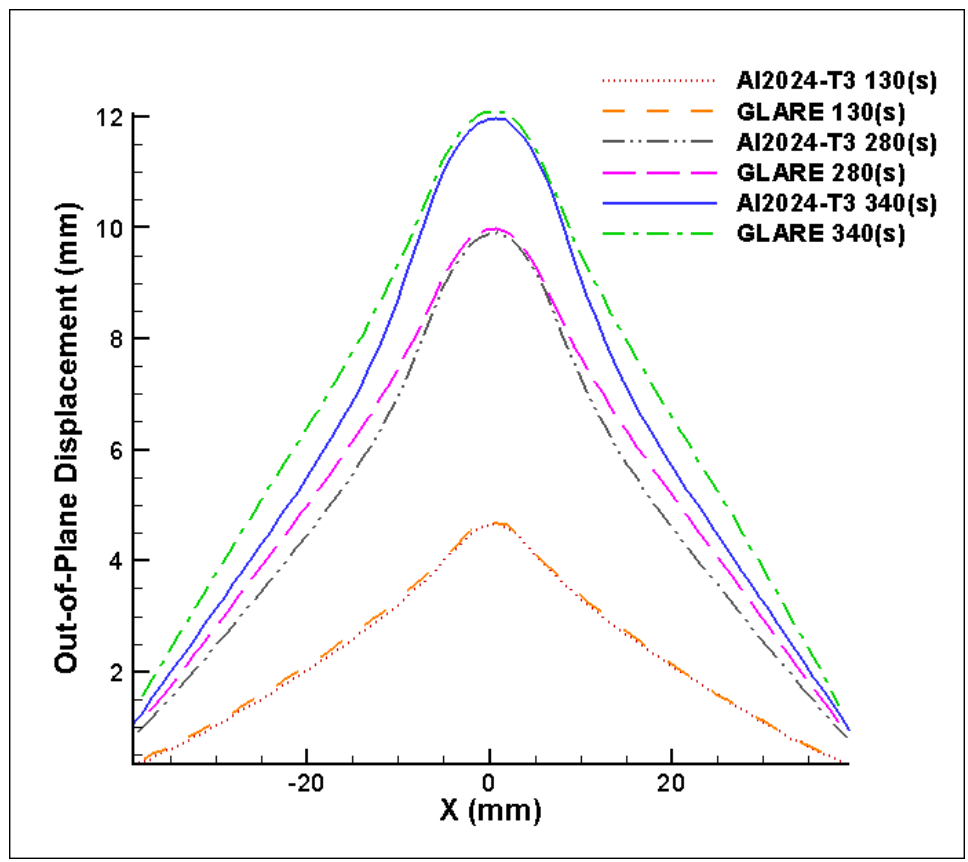

b.)

Figure 47: a.) out-of-plane displacement fringe plot for aluminum 2024-T3 at an indentation time of 320 (s) showing data extraction line b.) out-of-plane displacement profiles of GLARE and aluminum 2024-T3 at increasing indentation times 
Despite aluminum 2024-T3 reaching higher contact forces and impact energies before cracking in both LVI and QSI tests, the larger membrane deformations in GLARE could be beneficial in transferring loads to compliant supports. With greater membrane deformations the contact area between load transferring components and compliant supports would help to further exploit the energy absorption capabilities of the compliant support.

\subsubsection{Quantifying Visible Yield Damage}

Permanent visible damage caused by an impact event is an important failure limit when considering materials for numerous automotive applications [66]. For ductile metallic materials the first sign of visible damage is caused by yielding and the progression of plastic deformation zones. It has been proven in this work that a shortcoming of instrumented LVI tests is not being able to quantify and compare the plastic deformation that occurs in aluminum and FML specimens. An advantage of using DIC in QSI testing is that surface strain data is available to monitor and compare plasticity or permanent damage zones within specimens. With a stereoscopic DIC setup visible damage analysis can be taken a step further by overlaying strain fields on 3D Cartesian plots allowing for permanent dent depths to be related to plastic deformation. Using a yield strain of 0.004 [79] for aluminum 2024-T3, 3D plots of out-of-plane displacement with strain overlays are given in Figure 48 where the progression of plasticity zones in GLARE is demonstrated. Only 40 (s) into the QSI test yield has occurred on the aluminum panel furthest from indentation, but the yield zone is small and restricted to the contact area of the indenter. As the central deflection is approximately $1 \mathrm{~mm}$ at 40 (s) and the yield area is small there is not likely to be any permanent visible damage. At 100 (s) into the QSI 
test plasticity has progressed outside of the indenter-panel contact area and with a central deflection greater than $3 \mathrm{~mm}$ permanent visible damage has been created.

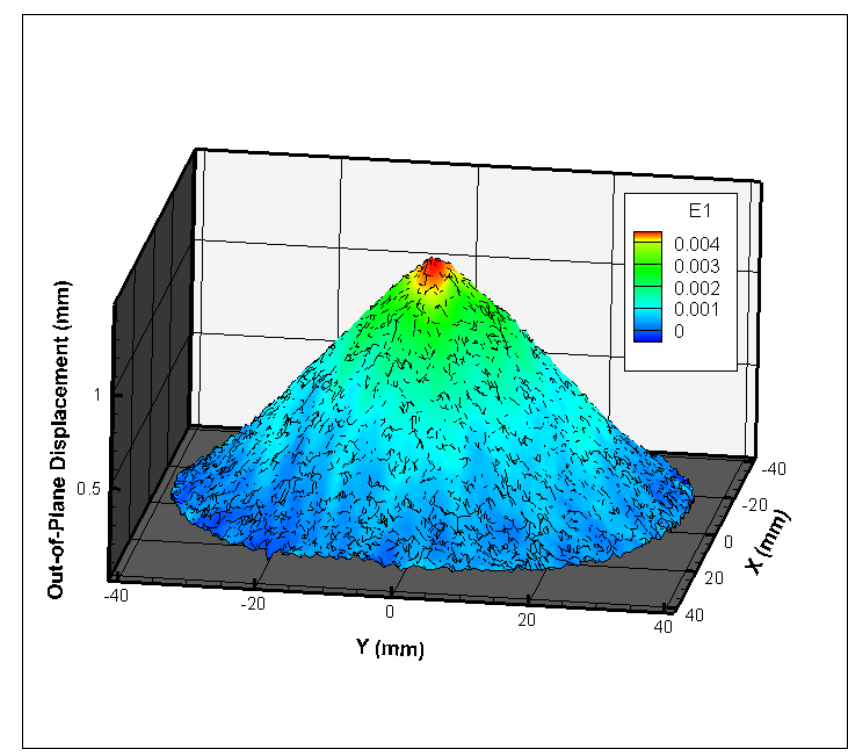

a.)

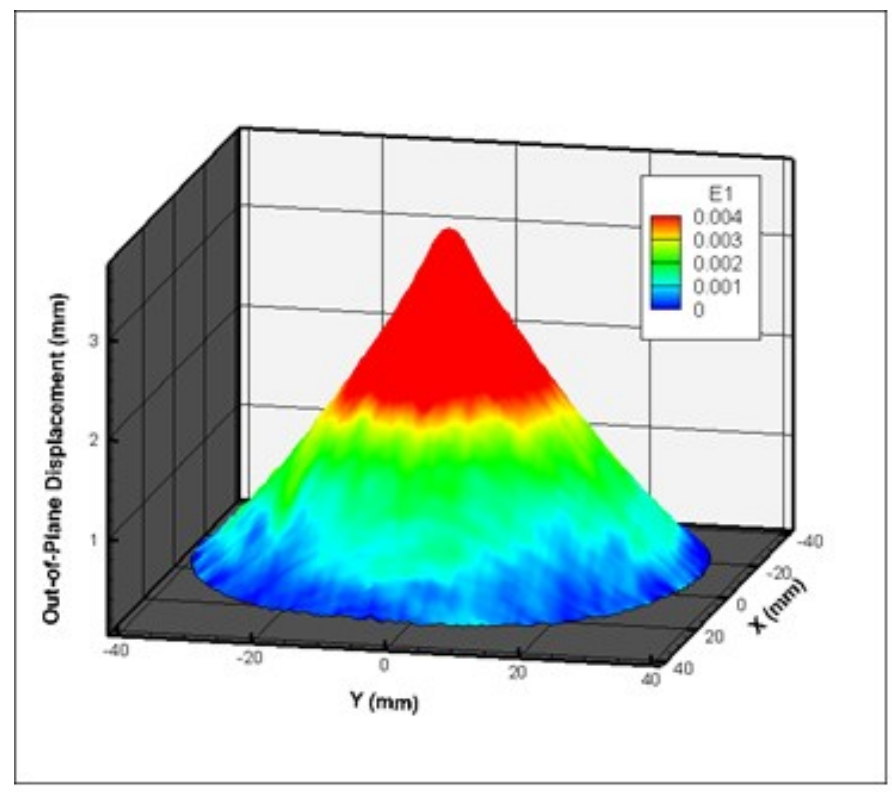

b.)

Figure 48: 3D Cartesian strain plots of GLARE depicting plastic deformation at a.) 40

(s) indentation time and b.) $100(\mathrm{~s})$ indentation time 
To quantify the amount of permanent visible damage the yielded area can be calculated from 2D strain plots as demonstrated in Figure 49 where the plasticity zones in aluminum 2024-T3 and GLARE at 70 (s) are shown in red. The plasticity zones at an indentation time of 70 (s) are almost identical between the two materials with GLARE having a slightly smaller yield area. In Figures 49 a.) and b.) the strain distribution is nonsymmetrical as higher maximum principal strains appear on the left side of the specimens. It is possible that the specimens were not placed right at the center of the circular QSI clamping fixture. However, this is most likely an effect of the difference in LED light intensity within the DIC setup where the light shining from right to left on the non-impact side of the panels is brighter.

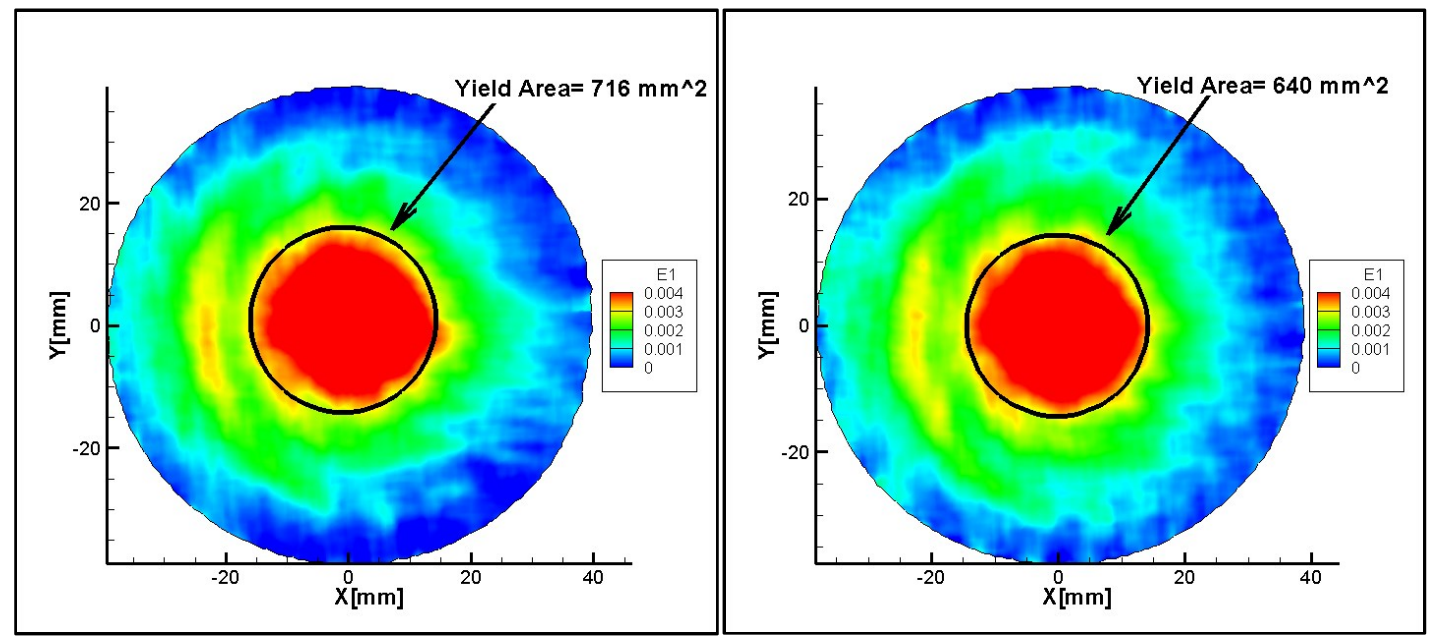

a.)

b.)

Figure 49: Maximum principal strain fringe plots at 70 (s) for a.) aluminum 2024-T3 and b.) GLARE

Under identical indentation time, all specimens shall have identical central deflection 
since the QSI test is displacement controlled. The composite cores within the FMLs create anisotropic responses compared to the isotropic responses of monolithic aluminum 2024-T3 panels. Therefore plastic areas will be different under identical indentation time between FML and aluminum 2024-T3 panels. A complete comparison for the progression of plastic deformation in GLARE and aluminum 2024-T3 samples is given in Figure 50 where for the majority of central deflection values the plastic areas are different between the two materials. Considering the controlling variables of interest for the visible damage failure limit are deflection and energy, the plastic area is given in terms of increasing central deflection along with curves identifying the indentation energy required.

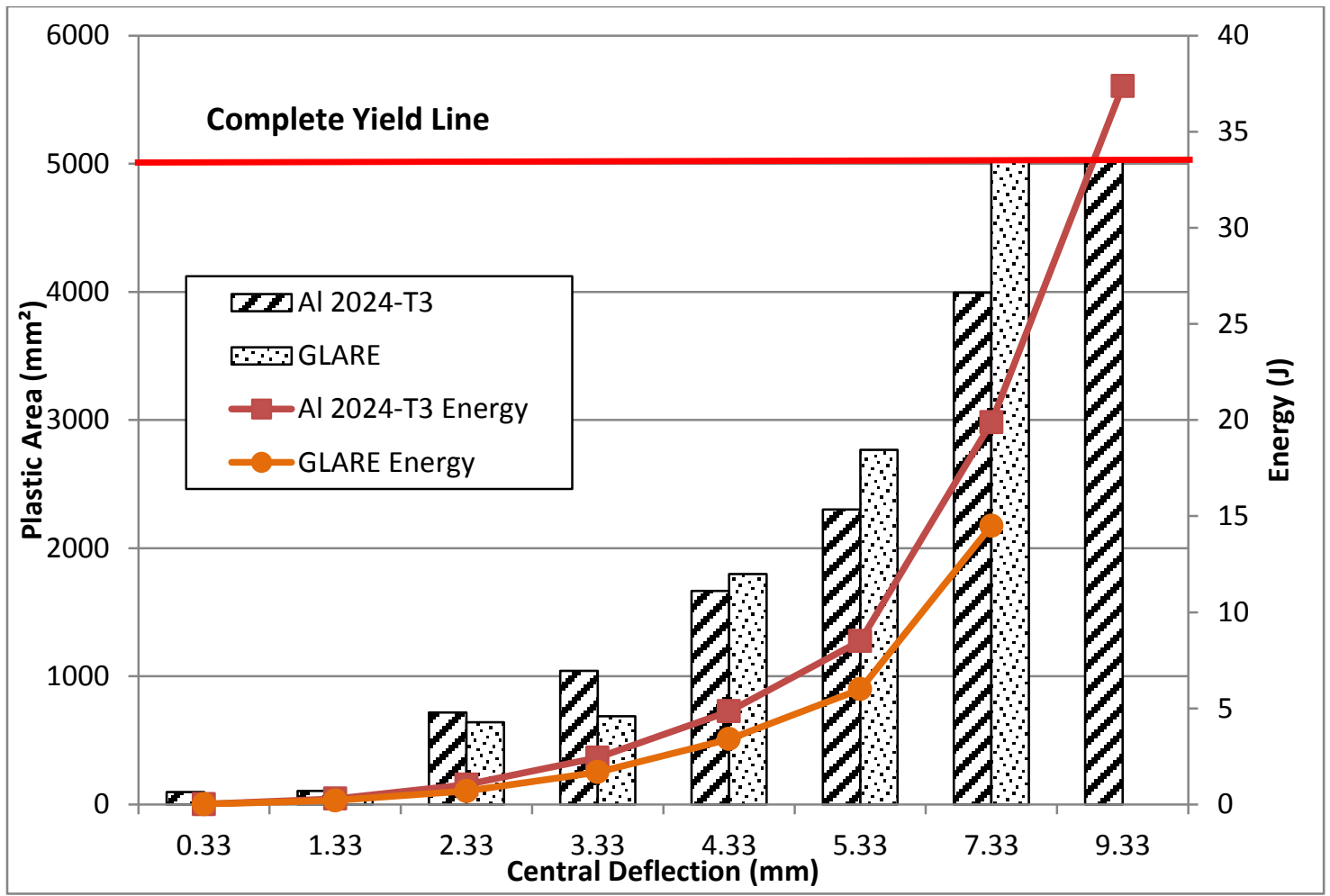

Figure 50: Progression of plastic deformation area in GLARE and aluminum 2024-T3 
At small central deflections up to $3.33 \mathrm{~mm}$ the yield area in GLARE is shown to be smaller than that in aluminum 2024-T3 and the indentation energies are almost identical. As the central deflection increases beyond $3.33 \mathrm{~mm}$ GLARE's plastic deformation area is greater at lower indentation energies than that of aluminum 2024-T3. Aluminum 2024-T3 also reaches full plasticity in the unsupported region at a central deflection $2 \mathrm{~mm}$ larger and at an indentation energy in excess of 20 joules more compared to GLARE. Yield and energy trends in Figure 50 are a direct result of strain hardening affects associated with plastic deformation in aluminum 2024-T3. As the yield area in aluminum 2024-T3 extends outside of the indenter-plate contact area the aluminum panel's stiffness increases. Since GLARE contains only a 53\% volume fraction of aluminum 2024-T3 it's stiffness does not increase as dramatically. This is supported by the force-deflection response of both materials in Figure 51 for QSI tests where at small deflections the stiffness (slope in force Vs. deflection curve) of each material is similar.

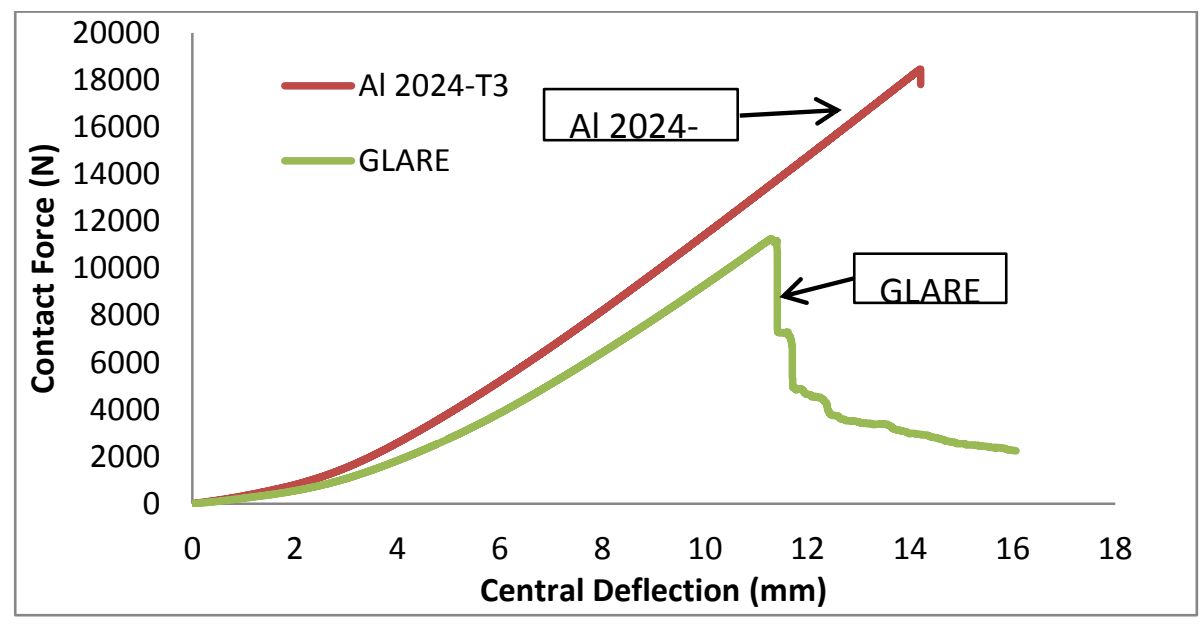

Figure 51: QSI force-deflection curves of aluminum 2024-T3 and GLARE taken to puncture 
However, beyond a deflection of approximately $2 \mathrm{~mm}$ the stiffness of the aluminum 2024-T3 panel is greater and therefore higher indentation energies and forces are required to progress the plastic deformation zone. These observations hold true for LVI tests that induce low strain rates as similar trends in aluminum 2024-T3 and GLARE panel stiffness are observed at low impactor velocities in Figure 52.

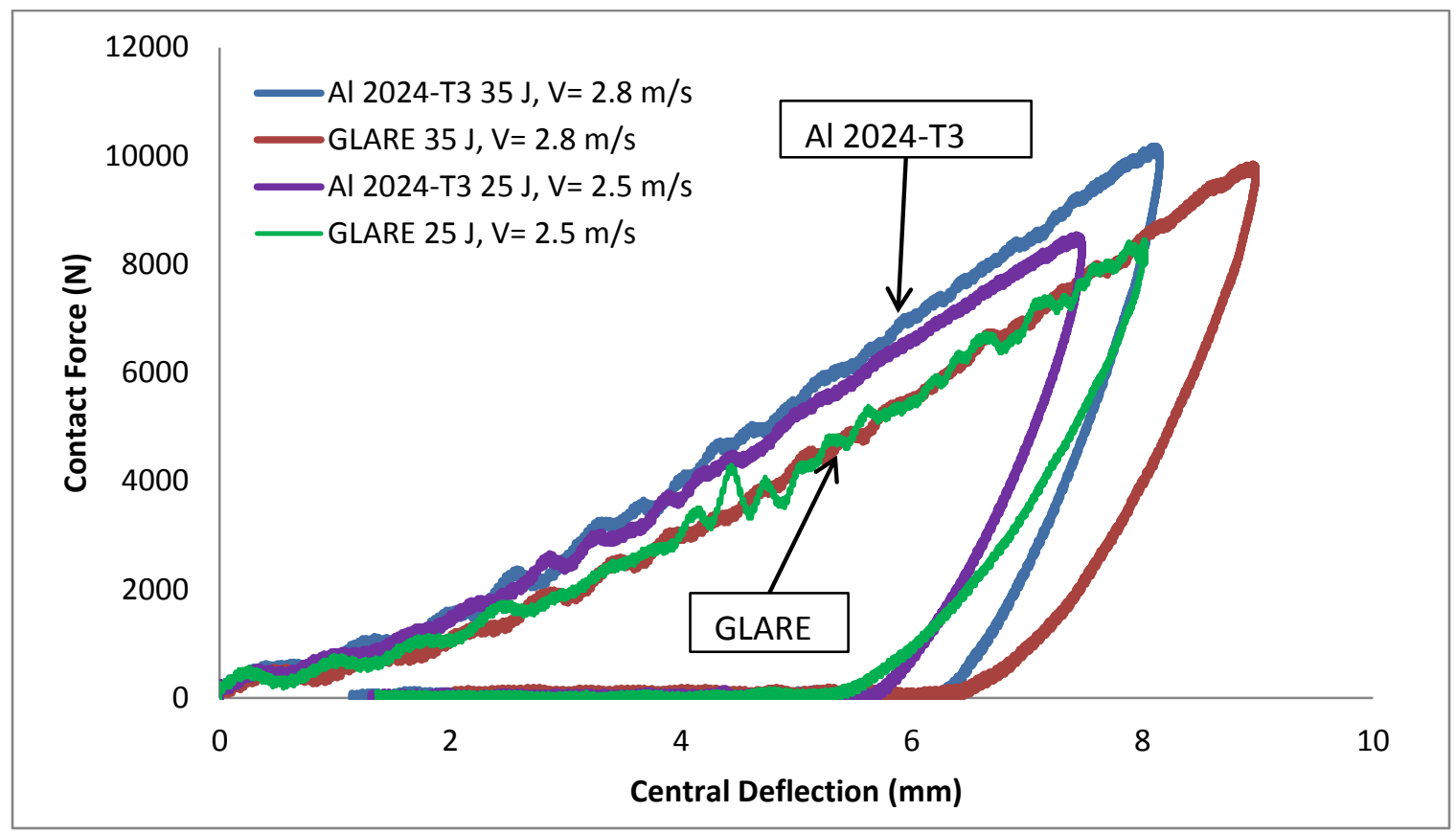

Figure 52: LVI force-deflection curves of aluminum 2024-T3 and GLARE at low impactor velocities

As impact velocity increases the strain rate dependent mechanical properties of glass fibre bring GLARE's stiffness closer to that of aluminum 2024-T3 throughout the entire impact event as shown in Figure 53. Thus the plastic damage regions are likely to be near identical at impactor velocities above $3 \mathrm{~m} / \mathrm{s}$. 


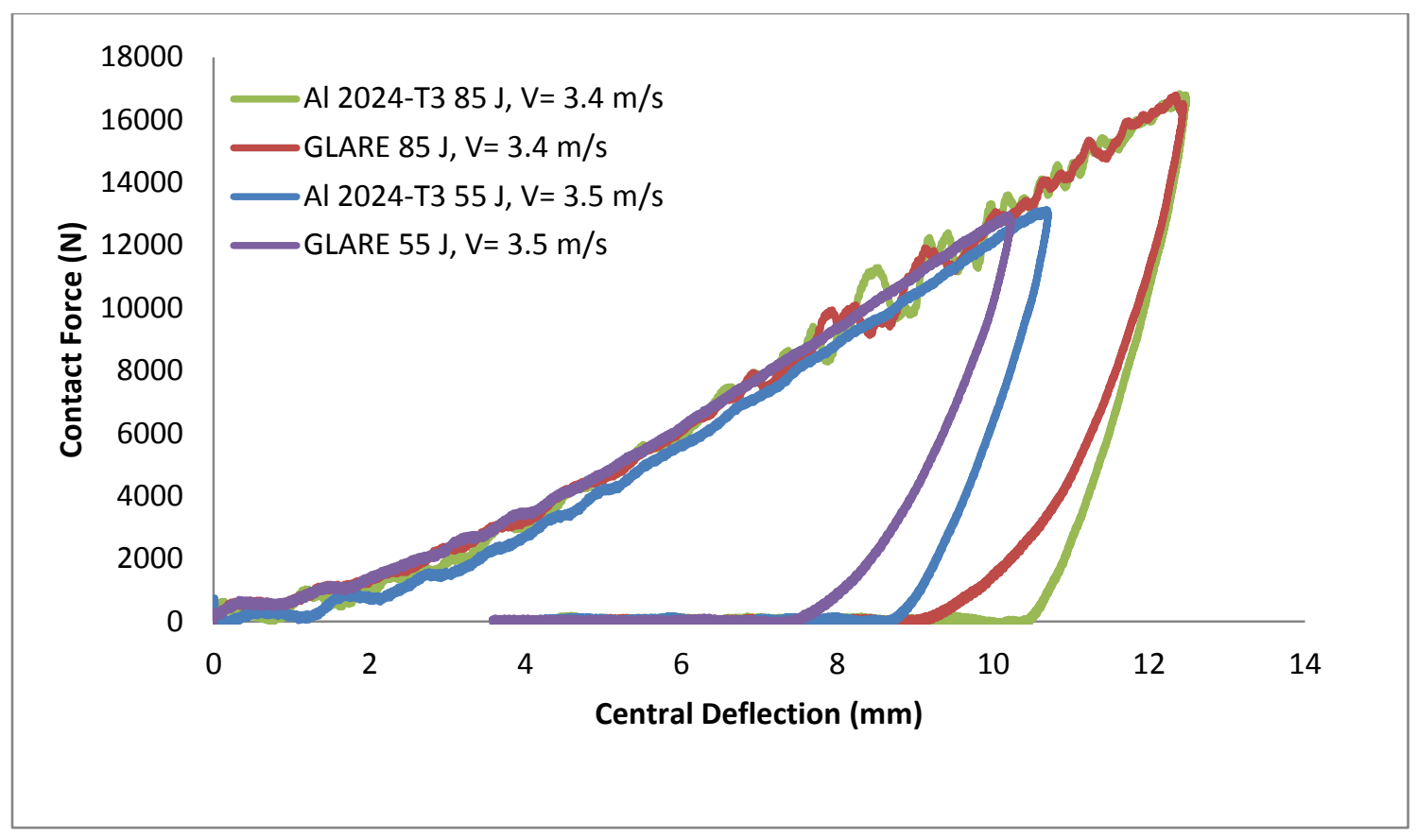

Figure 53: LVI force-deflection curves of aluminum 2024-T3 and GLARE at high impactor velocities

Figures 48-50 reaffirm earlier conclusions regarding quasi-static indentation tests being able to simulate low-velocity impacts. Furthermore, Figures 49 and 50 provide further evidence to suggest GLARE is a feasible lightweight alternative material option in impact prone structures as its force-deflection behaviour is close to matching that of aluminum 2024-T3.

\subsubsection{Strain Histories}

Through use of DIC technology in this research project full field strain data has enabled strain profile histories to be captured, crack initiation location and strain history to be documented, and a superior method for validating low-velocity FEA simulations. Strain histories were used in reference [24] to assist in describing the differences in low-velocity impact response among various grades of GLARE. In Figures 54 and 55 maximum 
principal strain profiles (data extracted along the same line as in Figure 47 a.)) are given for aluminum 2024-T3, GLARE, and CARAL captured during QSI testing. At an indentation time of 40 (s) (central deflection of $1.33 \mathrm{~mm}$ ) in Figure 54 there are only minor differences in the max principal strain among the materials as to be expected at a small indentation force. All materials are shown to have exceeded the yield strain of 0.004 for aluminum 2024-T3 and have interchangeable yield regions.

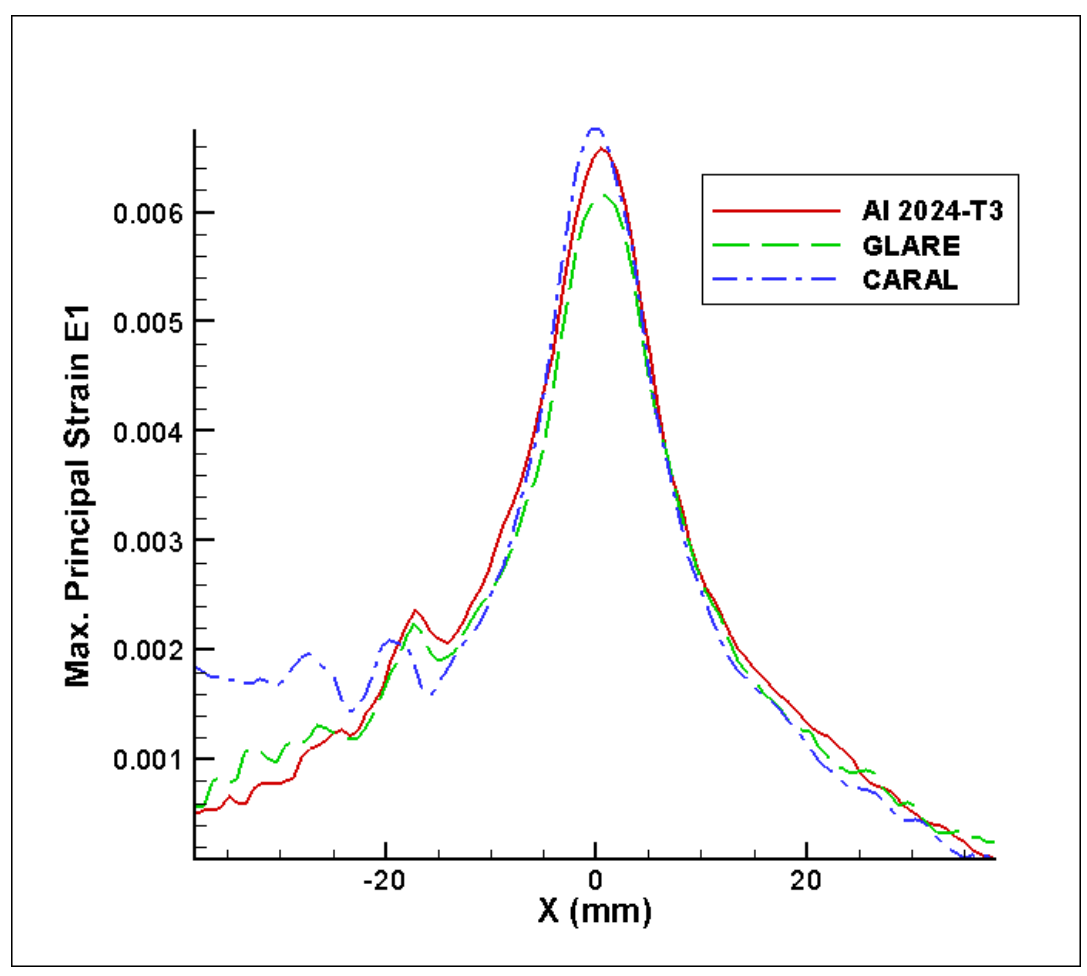

Figure 54: Maximum principal strain profiles at an indentation time of 40 (s)

In Figure 55 the maximum principal strain profiles are given just before a major crack develops on the non-impact side in each material. In low-velocity impact testing it is clear that a material's failure strain plays an important role in the force, deflection, and energy absorption achievable at break and total failure limits. The limits of carbon and 
glass fibre failure strains are expressed in Figure 55 based on the lower peaks of CARAL and GLARE strain profiles relative to that of aluminum 2024-T3 right before fracture.

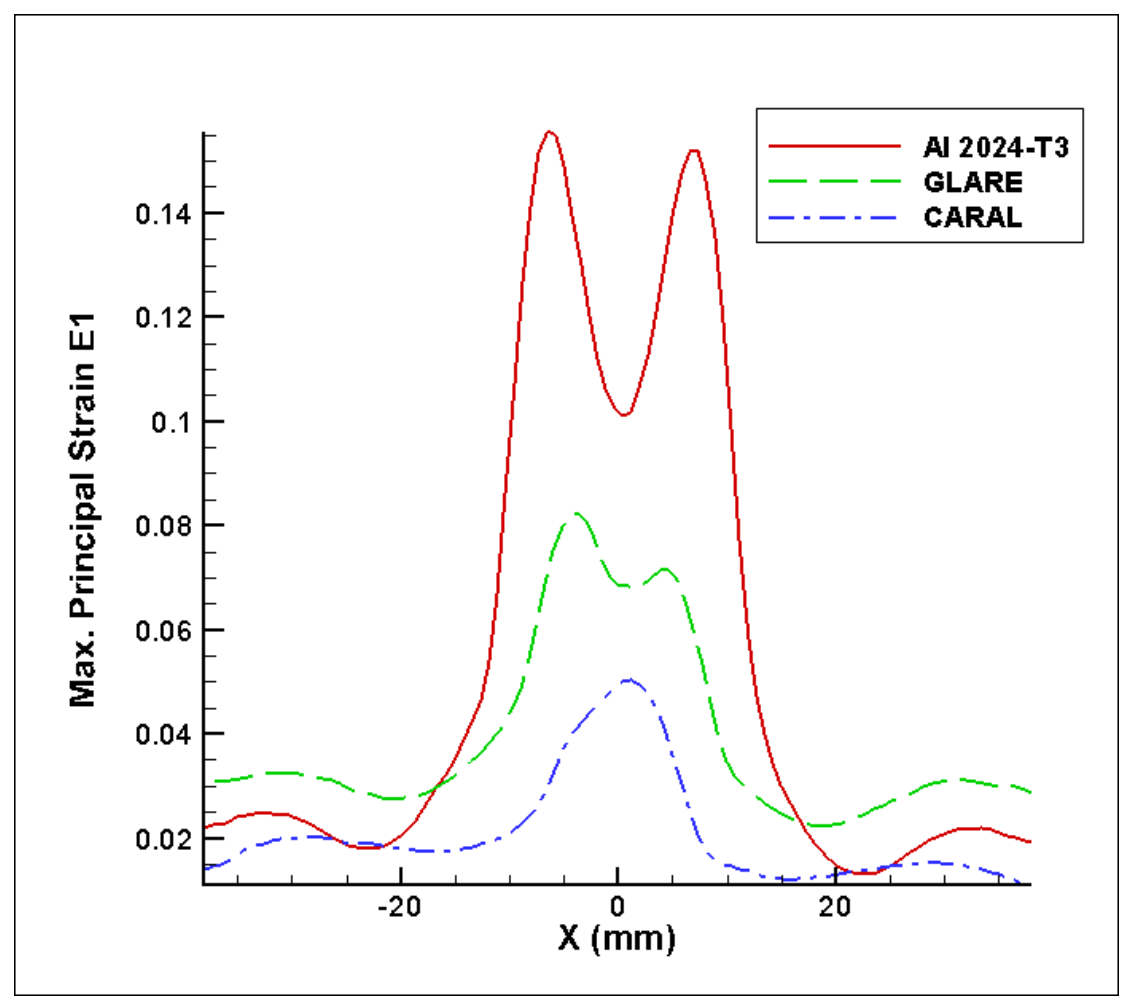

Figure 55: Maximum principal strain profiles just before crack initiation on the nonimpact side occurs

In the aluminum and GLARE specimens, friction between the indenter and panel is shown to play an important role in the strain profiles near fracture as local necking develops outside the center of the specimens. Sexton et al. [36] found similar results in conducting forming trials on aluminum and fibre metal laminates where it was proposed that interlaminar shear deformations reduce the effect of friction between the punch and FMLs. By contrasting strain profiles in Figure 56 of aluminum 2024-T3 and GLARE at large central deflections this appears to hold true for QSI testing as the peak strains in 
GLARE are less pronounced. Differences in frictional effects may also be due in part to discrepancies in cladded and non-cladded surfaces for the aluminum 2024-T3 in GLARE and the monolithic aluminum 2024-T3 respectively.

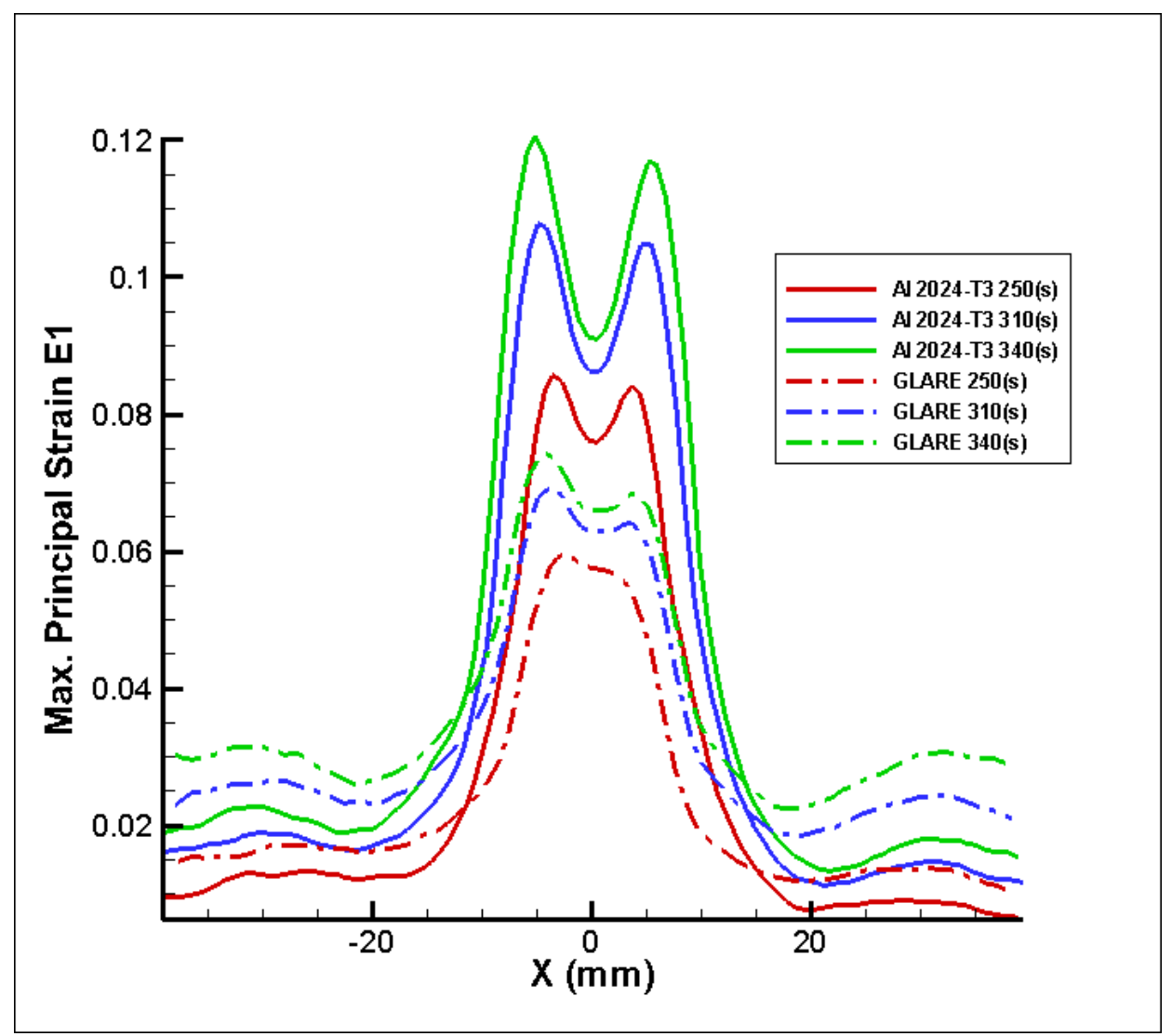

Figure 56: Max principal strain profiles at large central deflections for GLARE and aluminum 2024-T3 exposing differences in friction effects

The role of friction can be applied to LVI tests as damage states have been found to be comparable among the quasi-static indentation and low-velocity impact tests. Figure 57 demonstrates how the crack locations in LVI tested GLARE and aluminum 2024-T3 samples directly correspond to the high peripheral strains caused by friction in QSI tests. 
The only difference in frictional effects between the two tests would be the change from static to kinetic friction coefficient values for quasi-static to dynamic loading.

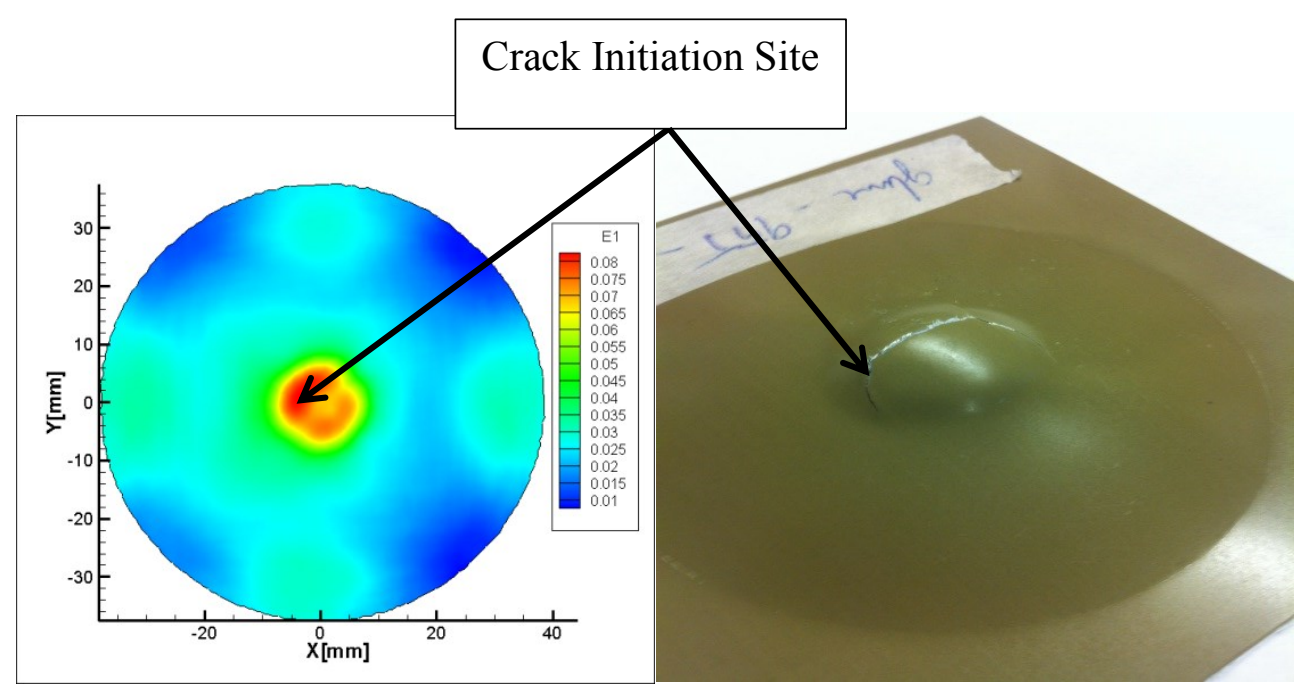
a.) QSI tested GLARE $\mathbf{E}_{\mathbf{1}}$ strain
b.) GLARE impacted at $95 \mathrm{~J}$ in LVI test

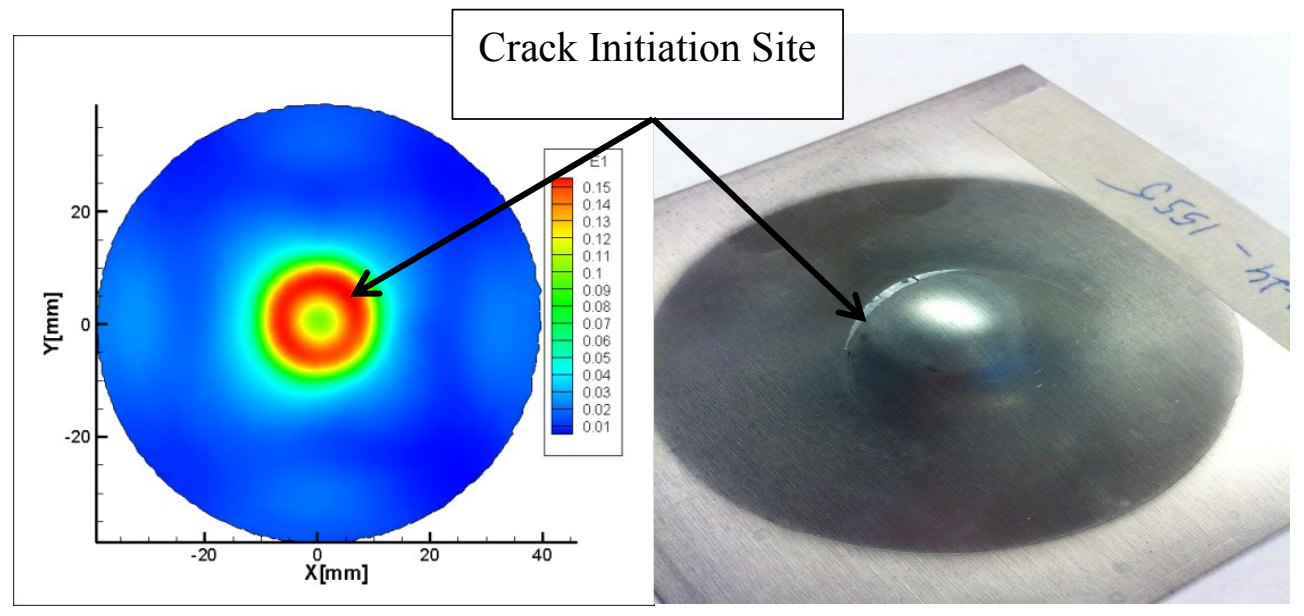

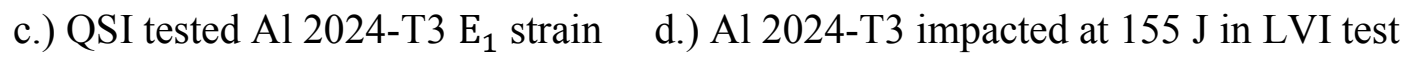

Figure 57: Correlating QSI E1 strain necking regions to crack locations on the nonimpact side of LVI tested samples 
Strain histories at the crack initiation site of GLARE are of particular interest because they can be used for design purposes. The in-plane surface strain paths at the center of the panel and at the crack initiation location are given in Figure 58 for the duration of the GLARE QSI test to better understand why the crack develops. In the center of the panel biaxial deformation is induced as maximum and minimum principal surface strains are equal. At the crack initiation location the in-plane strain path initially follows an approximate biaxial stretch until the frictional effects of the indenter-plate contact take over and result in a path approaching plane strain deformation [36]. Once the ratio of maximum to minimum principal surface strains reaches 1.3 the $\mathrm{S} 2$ glass fibres begin to break starting with the layer furthest from the indenter. This is indicated as a kink in the strain path at the crack initiation site as highlighted in Figure 58. Cracking in the aluminum 2024-T3 layers soon follow with the non-impact side cracking first.

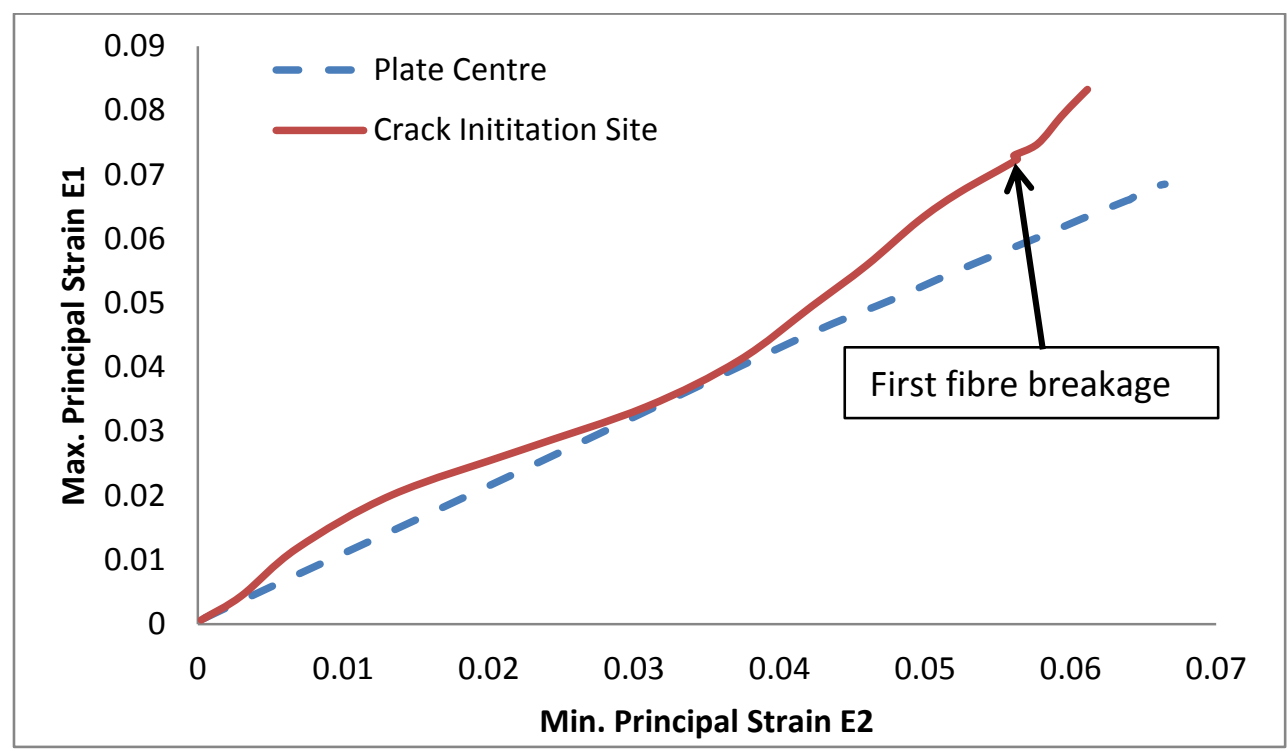

Figure 58: Strain paths in GLARE at the crack initiation site and at the centre of the panel 
The progression of failure at the crack location is better represented in Figure 59 where the evolution of maximum principal surface strain is plotted against indenter contact force. There is a clear distinction amid the first signs of S2 glass fibre breakage and the cracking of the non-impact aluminum 2024-T3 layer. At a maximum principal surface strain of 0.0723 in the outer aluminum layer of GLARE and at a load of $11.2 \mathrm{kN}$ the S2 glass fibres which are closest to the non-impact side fracture after which point the panel can no longer sustain an increase in load. For a brief period of time the load is sustained while S2 glass fibre breakage propagates through the thickness of the panel until a significant load drop signifies complete fracture of all S2 glass laminate layers. The final cracking of the non-impact side aluminum 2024-T3 layer then takes place followed by cracking of the impact side aluminum 2024-T3 layer.

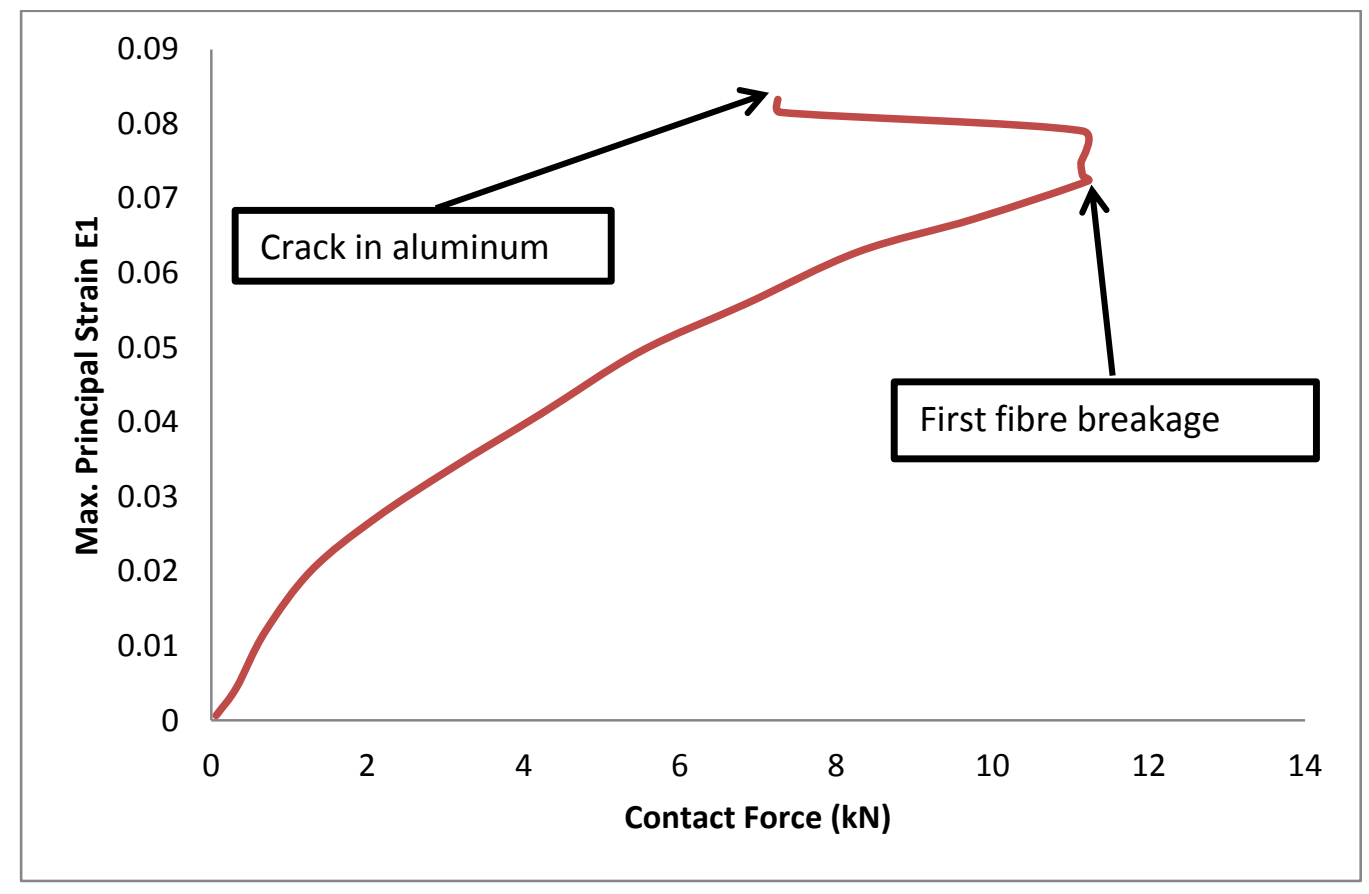

Figure 59: Progression of glass fibre and aluminum 2024-T3 failure in QSI tested GLARE 
With instrumented LVI testing multiple impacts would have to be performed to define the energy, force, and deflection to cause first signs of cracking. The implementation of DIC technology in QSI testing has allowed for a more precise method of defining the parameters at the first signs of cracking and for the glass fibre breakage that precedes it. Furthermore, having knowledge of load and surface strains at fibre and aluminum failure for out-of-plane loadings of GLARE is beneficial when designing and simulating impact prone structures. A restriction on the strain data obtained in this work is of course the use of quasi-static load application compared to the low-velocity impacts that would be seen in service for automotive components. As long as the velocities are relatively low and strain rates minimal in service GLARE strain histories will be similar to those presented here. If impact velocities are high enough to induce strain rate dependent behavior then GLARE will crack at higher loads and energies as determined from experimental LVI testing, the main difference being an increase in the failure strain of the S2 glass fibres. Thus the strain-load curve in Figure 59 for quasi-static loading conditions can be used as a worst case scenario in design.

A final advantage of obtaining full field DIC data is having the ability to contrast experimental strains at any point in the indented panel to those developed in low-velocity impact simulation models. Modeling the impact response of FMLs and composite materials is an extensive research area as numerous researchers have attempted to develop numerical and analytical models as described in references [26, 34]. Development of an accurate impact model for FMLs was not part of the current research project; however, a numerical low-velocity impact model of aluminum 2024-T3 was 
developed based on the work in reference [52]. Using this model brief observations could be made between experimental QSI and LVI simulation strain data. Figure 60 displays the max principal strain fringe plot from the finite element analysis impact simulation that can be referenced to the QSI strain field in Figure 57 c.).

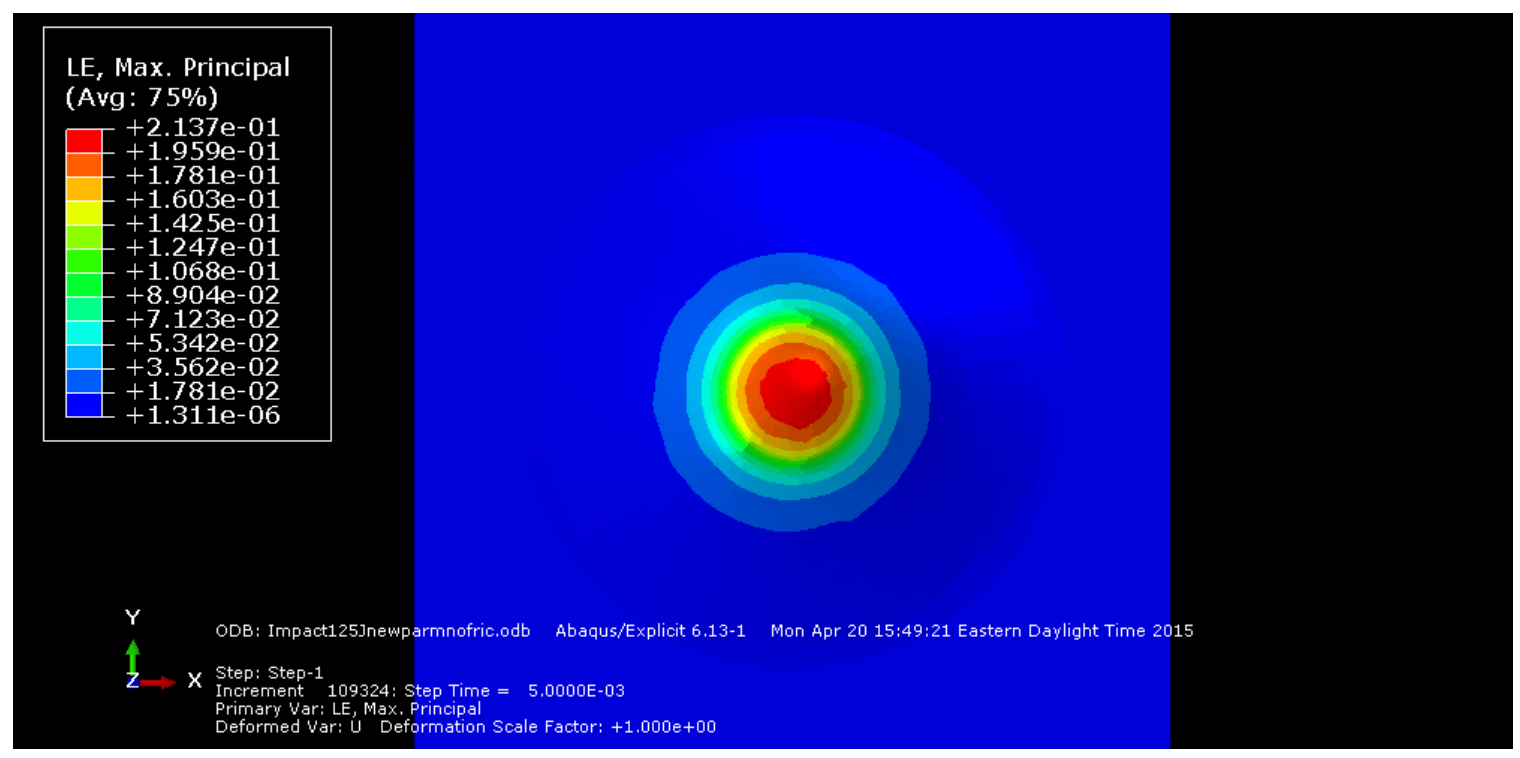

Figure 60: FEA analysis of aluminum 2024-T3 at an impact energy of $125 \mathrm{~J}$ without friction

It is evident that frictional effects are missing in the FEA simulation model since the maximum principal strain has not migrated outside the center of the plate. The inclusion of friction in the impact simulation of not only aluminum 2024-T3 but also GLARE FMLs is important as failure develops at the highly strained necking regions as discussed previously. Therefore static-kinetic friction behavior was added to the numerical model based on friction coefficients from reference [24]. As described by the general contact algorithm in Abaqus, the friction between the aluminum 2024-T3 and impactor was 
introduced tangentially. Therefore the effects of friction increase as the plate forms around the impactor's hemispherical head. The addition of tangential friction has led to better agreement between strain histories from the QSI test and FEA simulation as shown in Figure 61 . The strain history of position $(7,0)$ represents the max principal strain necking region in Figure 57 c.).

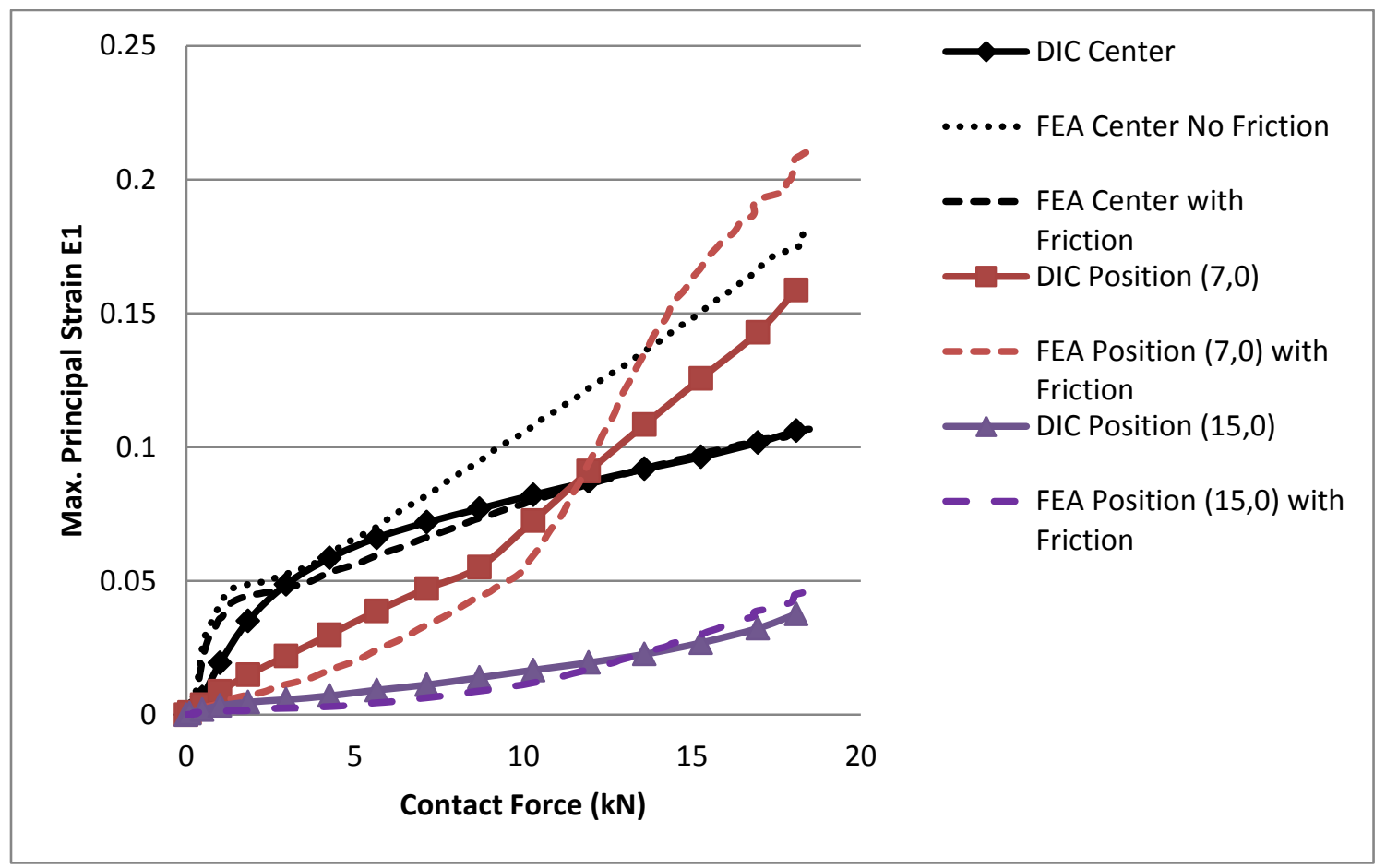

Figure 61: Comparison of max. principal strains at different (x,y) positions between DIC indentation tests and a 125 joule FEA impact model 


\section{Conclusions and Future Work}

\subsection{Conclusions}

The research presented in this thesis assessed the low-velocity impact performance of carbon/epoxy, carbon/nylon, CARAL and GLARE lightweight materials against a baseline aluminum 2024-T3 alloy. Low velocity impact performance was evaluated using 1) instrumented drop weight and 2) QSI tests. The ability to use QSI tests to replicate low-velocity impact responses was demonstrated and the use of DIC for performance assessment was presented.

The conclusions drawn from this thesis are:

- Based on relevant impact performance parameters for automotive applications aluminum 2024-T3 was found to perform better in impact than the lightweight materials considered in this study. More specifically, aluminum 2024-T3 was superior in its ability to sustain load before puncturing and had the ability to absorb more energy per unit mass. Strain rate strengthening mechanisms of the glass fibres in the GLARE FML set it apart from the other lightweight materials impacted and gave it deflection capabilities similar to aluminum 2024-T3. It was also determined that the impact characteristics of each material matched their respective stress-strain responses especially when comparing strain energy density to energy absorption achieved.

- In ranking materials for automotive applications, limitations were discovered with using instrumented drop weight test data. This included the inability to define or 
quantify visible damage in aluminum 2024-T3 and FML samples where plastic deformation occurs. Also, there is a lack of global deformation knowledge as the deflection is only given for one point in the plate.

- An extensive correlation of QSI and LVI test data exposed the limitations of quasi-static loading to produce similar damage states and results to dynamic impacts. QSI tests were unable to replicate low-velocity impact responses in carbon composite panels. The inclusion of ductile aluminum layers to carbon/epoxy in CARAL resulted in good comparability between the two tests, while monolithic aluminum 2024-T3 also had good correlation as its strain rate effects were proven to be minimal. Strain rate effects in GLARE were determined to be more prominent and thus QSI tests are only efficient at simulating dynamic impact events when the impactor velocities are kept to a minimum. In this research the limit was approximately $3 \mathrm{~m} / \mathrm{s}$.

- A methodology was developed for approaching and verifying stereovision DIC setups that is not limited to DIC use in quasi-static indentation tests. Verification of DIC strain fields with a fibre optic strain gauge gave full confidence in experimental results.

- Full field DIC data was proven to offer many advantages in the assessment of a material's impact performance as out-of-plane displacement profiles provided evidence that larger membrane deformations exist in GLARE than in aluminum 2024-T3. DIC strain data also offered a means to quantify and compare the progression of visible yield damage in GLARE and aluminum 2024-T3 plates which was a major disadvantage in LVI testing. Further analysis outcomes only 
available with DIC data were the precise mapping of strain at the crack initiation site in GLARE which is valuable for design. The DIC data also clearly demonstrated the effects of indenter-plate friction on the evolution of strain in an impact sample. This encouraged the implementation of friction in an FEA lowvelocity impact model where full field DIC data allowed for direct comparisons to be made at any point on the experimental model.

In considering the first research objective, the lightweight materials tested in this study were not able to match the impact performance of monolithic aluminum 2024-T3 when focusing on functional requirements for automotive applications. With regards to the original research question and objective in evaluating QSI testing as a means for qualifying and comparing potential materials in the automotive industry, the following can be said. QSI can be a useful tool in evaluating materials, but it has its limitations. As observed in this study, different damage mechanisms, intrinsic material properties, and material sensitivities contribute to the overall LVI behaviour. QSI testing can help evaluate some of these, but how they translate to LVI can vary from case to case. Given the simplicity and the amount of potential information that can be drawn from QSI testing, it serves as an excellent starting point in evaluating potential new materials. However, such testing should be followed up with at least partial LVI or other representative dynamic impact testing in order to identify potential limitations in QSI testing results. Furthermore, the incorporation of DIC technology enhances the usefulness of QSI tests as full field displacement and strain data reveals important considerations for design and simulation. 


\subsection{Future Work}

A standard GLARE 5 2/1-0.3 fibre metal laminate has shown to possess optimal impact performance over other lightweight materials with the exception of monolithic aluminum 2024-T3. If GLARE is to be considered for use in automotive applications it must produce similar impact characteristics to monolithic aluminum sheet as automakers strive to maintain current vehicle functionalities and levels of vehicle safety. Future work should thus focus on optimizing the glass-fibre and aluminum layer configurations within the GLARE FML with an emphasis on increasing its break resistance and energy absorption capacity. Enhancing the impact characteristics of the GLARE must be accomplished without compromising its weight saving advantages over monolithic aluminum sheet. In terms of experimenting different constituents in FMLs, it is proposed that the combination of aluminum layers with a thermoplastic composite will produce better impact performance. This is based on the observation of carbon/nylon attaining similar impact resistance to that of CARAL. In developing FMLs for automotive applications greater focus should also be placed on using less expensive composite constituents and avoiding costly carbon and S2 glass fibre composites. Furthermore, to bring impact studies more in line with the automotive industry potential lightweight materials should be compared with either 5000 or 6000 series automotive aluminum alloys. Replacement of aluminum 2024-T3 layers in GLARE with a 5000 or 6000 series aluminum alloy should also be considered. 
The practice of employing full field DIC data in the analysis of a materials impact performance should be expanded in future work to cement the advantages proposed in this study. It is suggested that a stereovision DIC setup be implemented in low-velocity impact tests to avoid the limitations of quasi-static loads in reproducing dynamic impact response. Of particular interest would be capturing full field DIC data during the high strain rate induced impact response of GLARE at greater impactor velocities. 


\section{Appendices}

\section{Appendix A Non-Destructive Inspection of Manufactured Panels}

Figure A1 presents the $\mathrm{C}$-scan results for the GLARE and CARAL panels, while figures $\mathrm{A} 2$ and $\mathrm{A} 3$ show the as manufactured panels with defects outlined in black marker. No major defects were present in the $\mathrm{C}$-scan images.

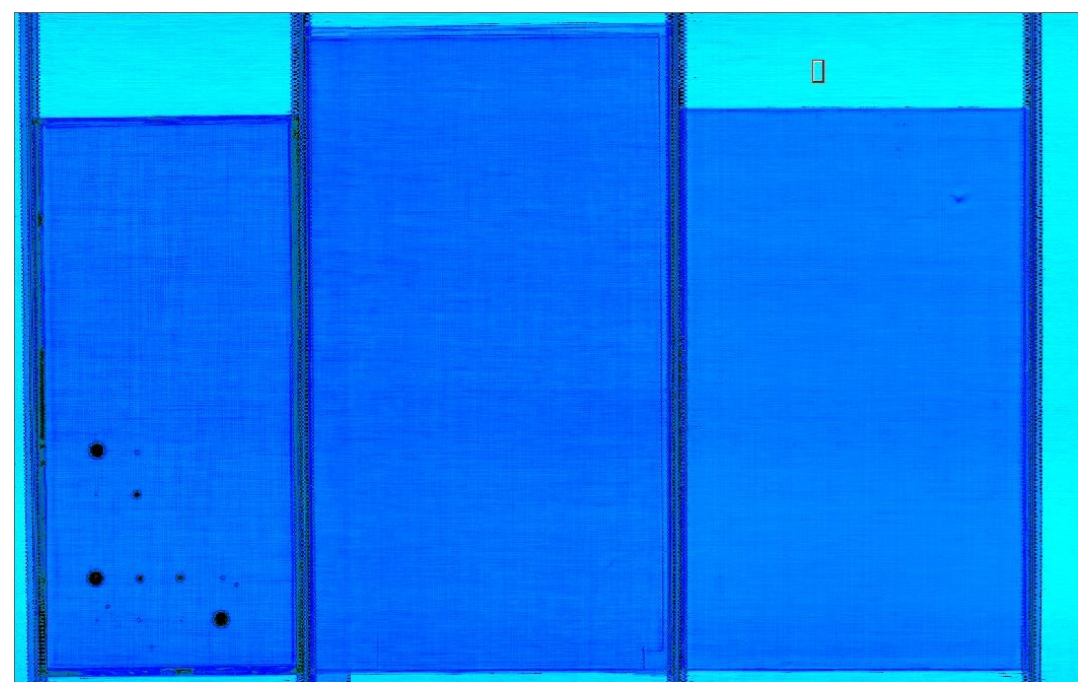

Figure A1: C-scan image of (far left) GLARE reference panel, (middle) GLARE panel, and (far right CARAL panel) 


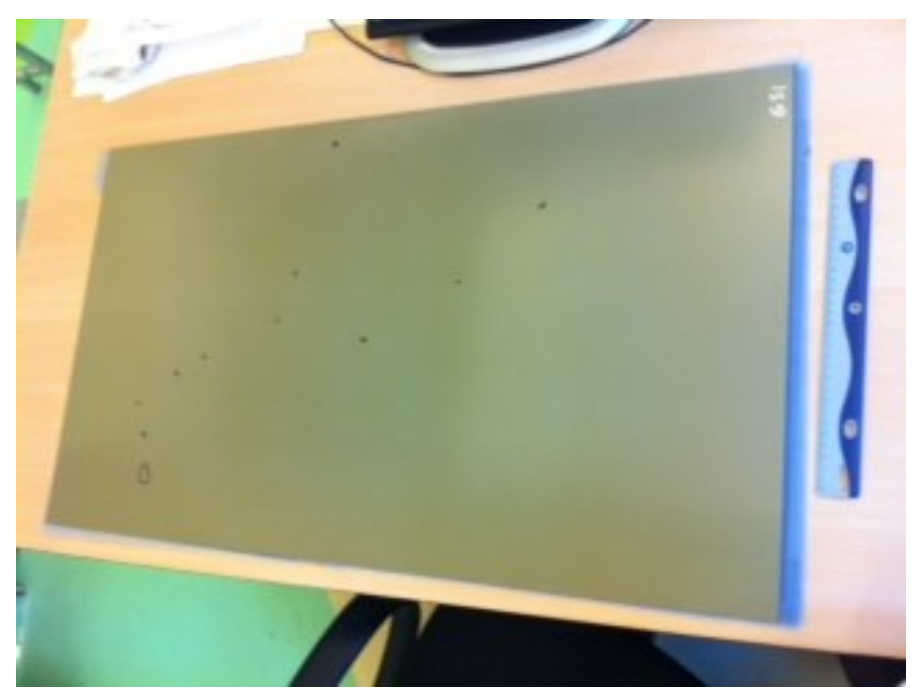

a.)

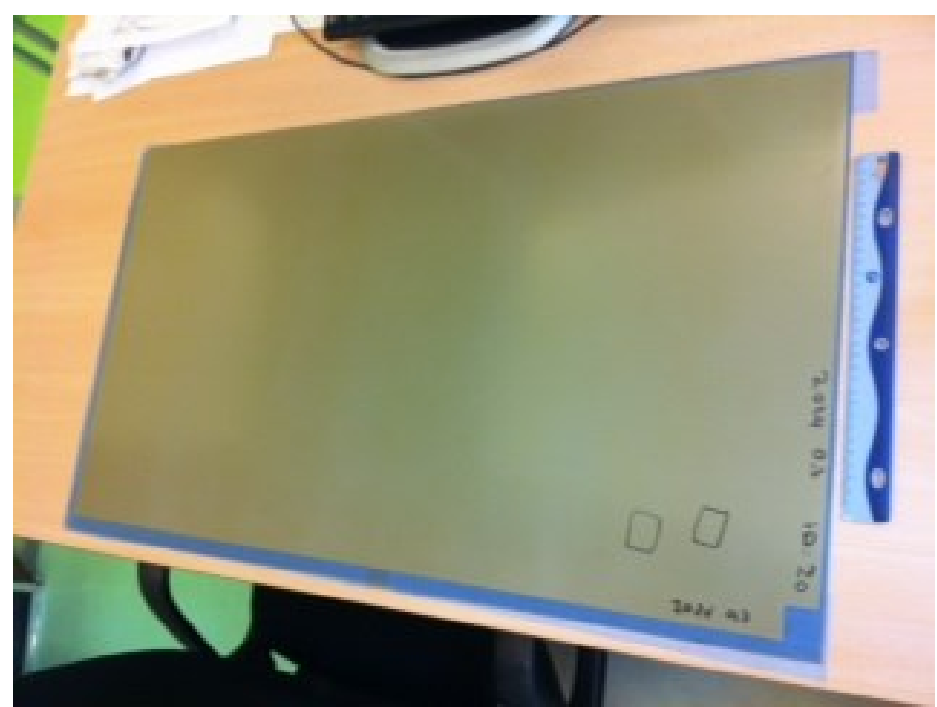

b.)

Figure A2: a.) Top side of GLARE panel with marked defect areas b.) underside of GLARE panel with marked defect areas (30 $\mathrm{mm}$ ruler shown for scale) 


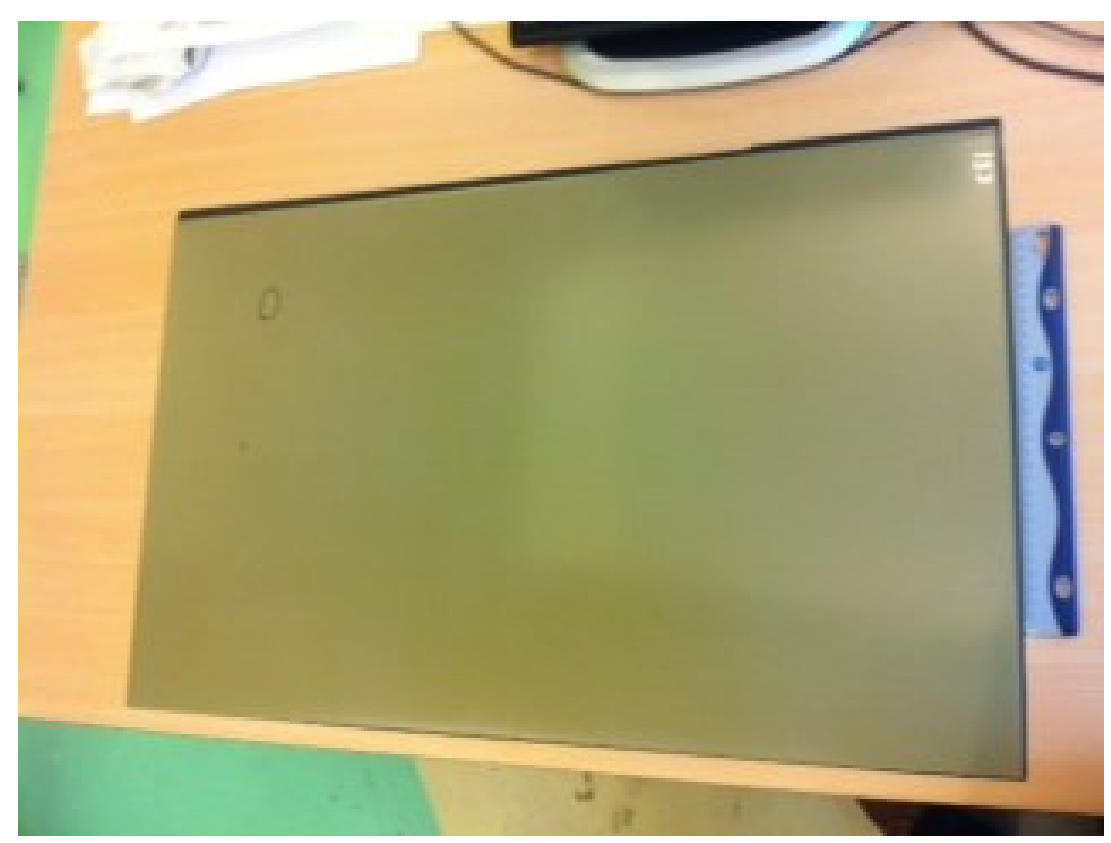

a.)

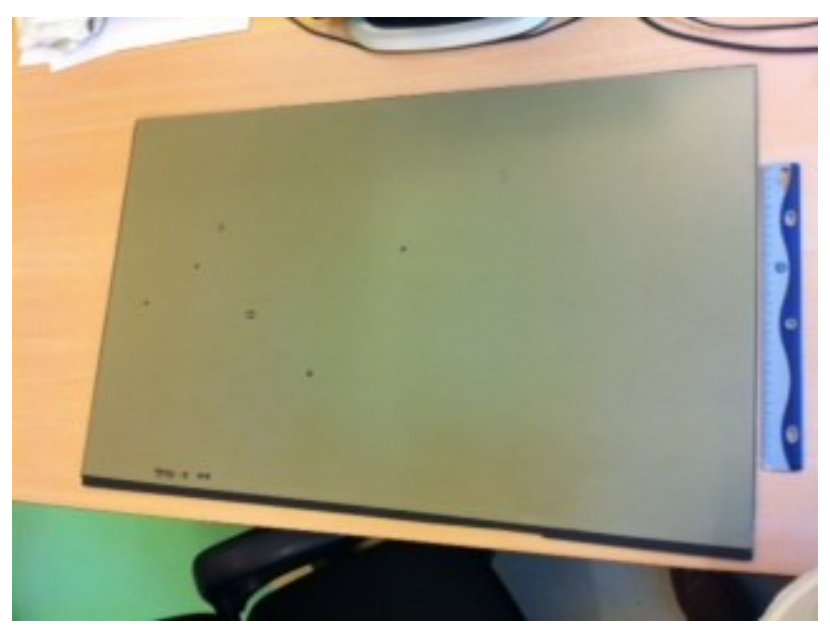

b.)

Figure A3: a.) Top side of CARAL panel with marked defects b.) underside of CARAL panel with marked defects (30 $\mathrm{mm}$ ruler shown for scale)

Most defects found in the C-scan and those that are outlined by black marker in figures A2 and A3 are in the form of permanent dents on the aluminum sheets. Since the 
aluminum used in manufacturing is very thin $(0.3 \mathrm{~mm})$, it dents/scratches very easily during transport. Thus the defects are not within the composite layers themselves.

Figure A4 is a C-scan image of the carbon/epoxy panel while Figure A5 shows the as manufactured panel with the defects outlined with silver marker.

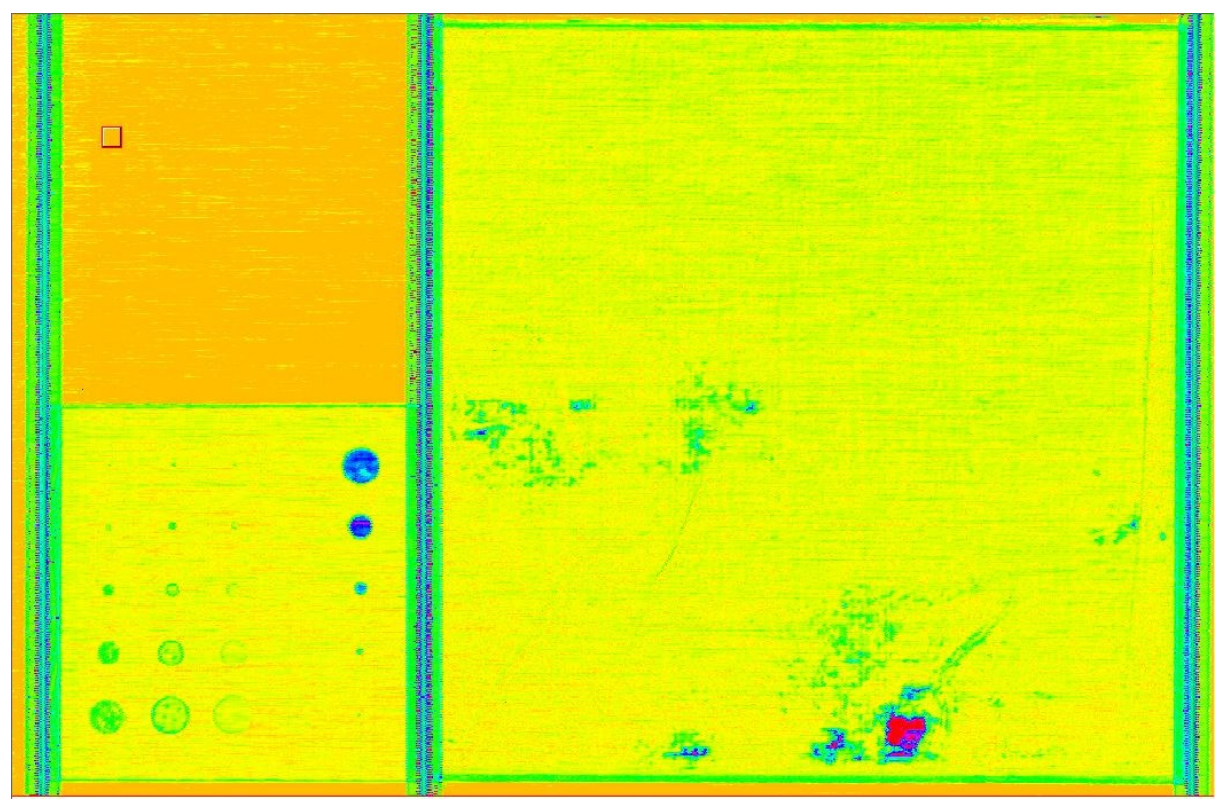

Figure A4: C-scanned image of (to the left) carbon/epoxy reference panel and (to the right) CARAL panel

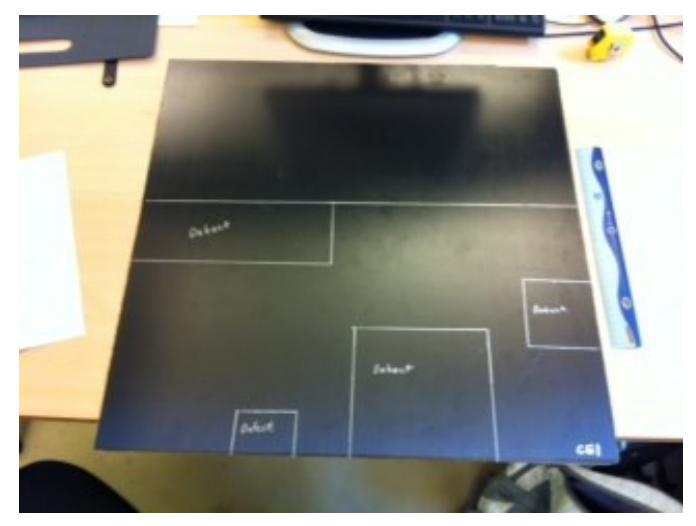

Figure A5: Carbon/epoxy panel with defect areas outlined (30 $\mathrm{mm}$ ruler shown for scale) 
The carbon/epoxy panel contains some areas with major defects due to poor manufacturing techniques with pre-preg rolls of carbon/epoxy which tends to wrinkle and form air pockets very easily during lay-up. As Figure A5 shows these areas have been marked on the as manufactured panel to avoid their inclusion in the test specimens. Figure $\mathrm{A} 6$ represents the $\mathrm{C}$-scan images of the thermoplastic panel carbon/nylon, for which there are no major defects on the majority of the panel except for in some corners which will be neglected for testing.

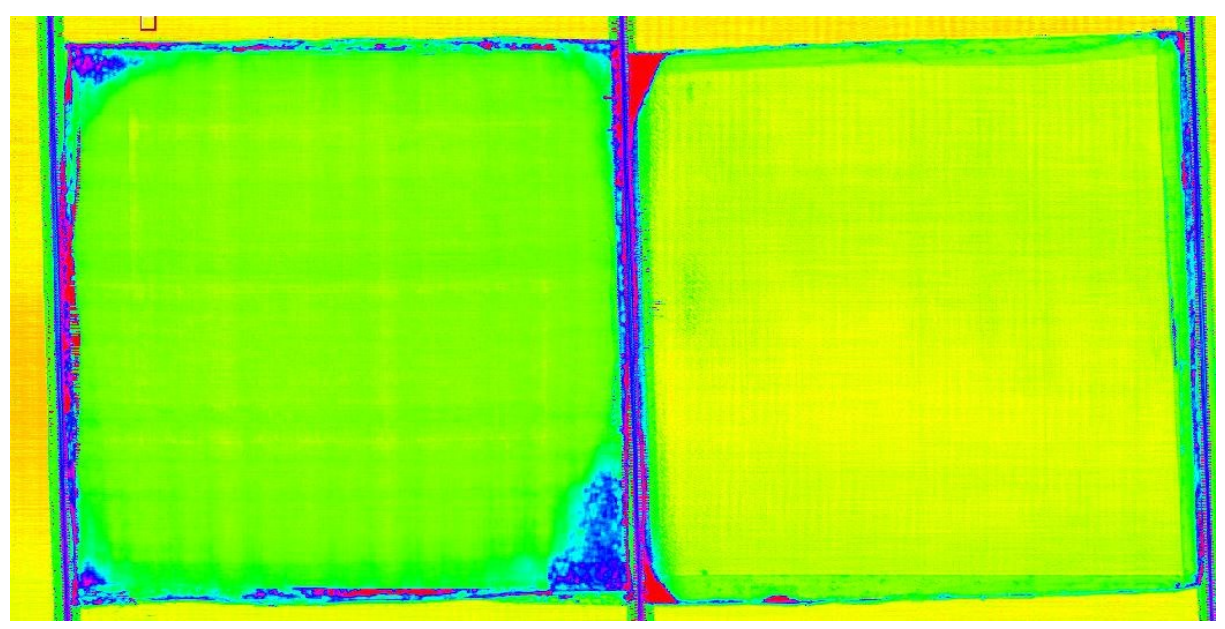

Figure A6: C-scan image of carbon/nylon panel (on the left) 


\section{Appendix B Low-velocity Impact Setup Information}

Table B1: Impactor mass and velocity at each impact energy tested

\begin{tabular}{|c|c|c|}
\hline Impact Energy (J) & Impact Mass (kg) & Impactor Velocity $(\mathrm{m} / \mathrm{s})$ \\
\hline 2 & 5.4487 & 0.74 \\
\hline 5 & 8.6487 & 1.07 \\
\hline 12.55 & 8.6487 & 1.69 \\
\hline 15 & 8.6487 & 1.83 \\
\hline 25 & 8.6487 & 2.40 \\
\hline 35 & 8.6487 & 2.83 \\
\hline 45 & 8.6487 & 3.23 \\
\hline 55 & 8.6487 & 3.56 \\
\hline 65 & 8.6487 & 3.80 \\
\hline 75 & 8.6487 & 4.20 \\
\hline 85 & 15.0287 & 3.37 \\
\hline 95 & 15.0287 & 3.56 \\
\hline 105 & 15.0287 & 3.74 \\
\hline 125 & 16.0987 & 3.94 \\
\hline 135 & 16.0987 & 4.10 \\
\hline 145 & 16.0987 & 4.24 \\
\hline 155 & 16.0987 & 4.4 \\
\hline
\end{tabular}




\section{Appendix C DIC Terminology and Parameters}

Processing DIC results involves reporting set and measured parameters that may have an effect on displacement and strain results. Important parameters may be reported in Table format and are outlined in Table $\mathrm{C} 1$. In Table $\mathrm{C} 1$ each parameter is listed as set or calculated based on how it is obtained during testing. 
Table C1: DIC reporting Table

\begin{tabular}{|c|c|}
\hline Technique Used & Stereovision Digital Image Correlation \\
\hline Noise Camera 0 & Measured \\
\hline Noise Camera 1 & Measured \\
\hline Pre-filtering & Set in software \\
\hline Subset & Set in software \\
\hline Step & Set in software \\
\hline Correlation Criterion & Set in software \\
\hline Shape Function & Set in software \\
\hline Interpolation Function & Set in software \\
\hline Measurement Points & Set in software \\
\hline Total Number of Images & Determined by user pre-test \\
\hline \multicolumn{2}{|l|}{ Displacement: } \\
\hline Spatial Resolution & Calculated by user \\
\hline In-plane resolution & Calculated by user \\
\hline Out-of-plane resolution & Calculated by user \\
\hline \multicolumn{2}{|l|}{ Strain: } \\
\hline Smoothing Method & Set in software \\
\hline VSG & Calculated by user \\
\hline Spatial Resolution & Calculated by user \\
\hline Resolution & Calculated by user \\
\hline
\end{tabular}


Many of the parameters are set in the software and it is acceptable in most cases to use the default settings for data processing. However, parameters such as noise, displacement and strain resolutions, and VSG (virtual strain gauge) must be calculated by the user and reported for each test. It is also important the user identifies the subset and step sizes used for DIC data processing as these can have a significant effect on the results obtained.

\section{Definitions of Important DIC Parameters and How They Can Affect the Data}

The following is a brief review of definitions for important DIC parameters as well as quantitative evidence of how these parameters might affect the DIC data. The information in this section is solely based on the DIC course Metrology Beyond Colours offered by the University of Leuven and MatchID.

\section{Camera Noise:}

The noise in each camera for a stereovision setup may be evaluated within the DIC software as a standard deviation percentage and should be reported for each test as higher noise levels will have more significant effects on the data. Thus static reference images should be taken before loads are applied to evaluate the noise levels in each camera. Figure $\mathrm{C} 1$ demonstrates how increasing noise levels cause an increase in displacement standard deviations or resolutions for the same un-deformed images. 


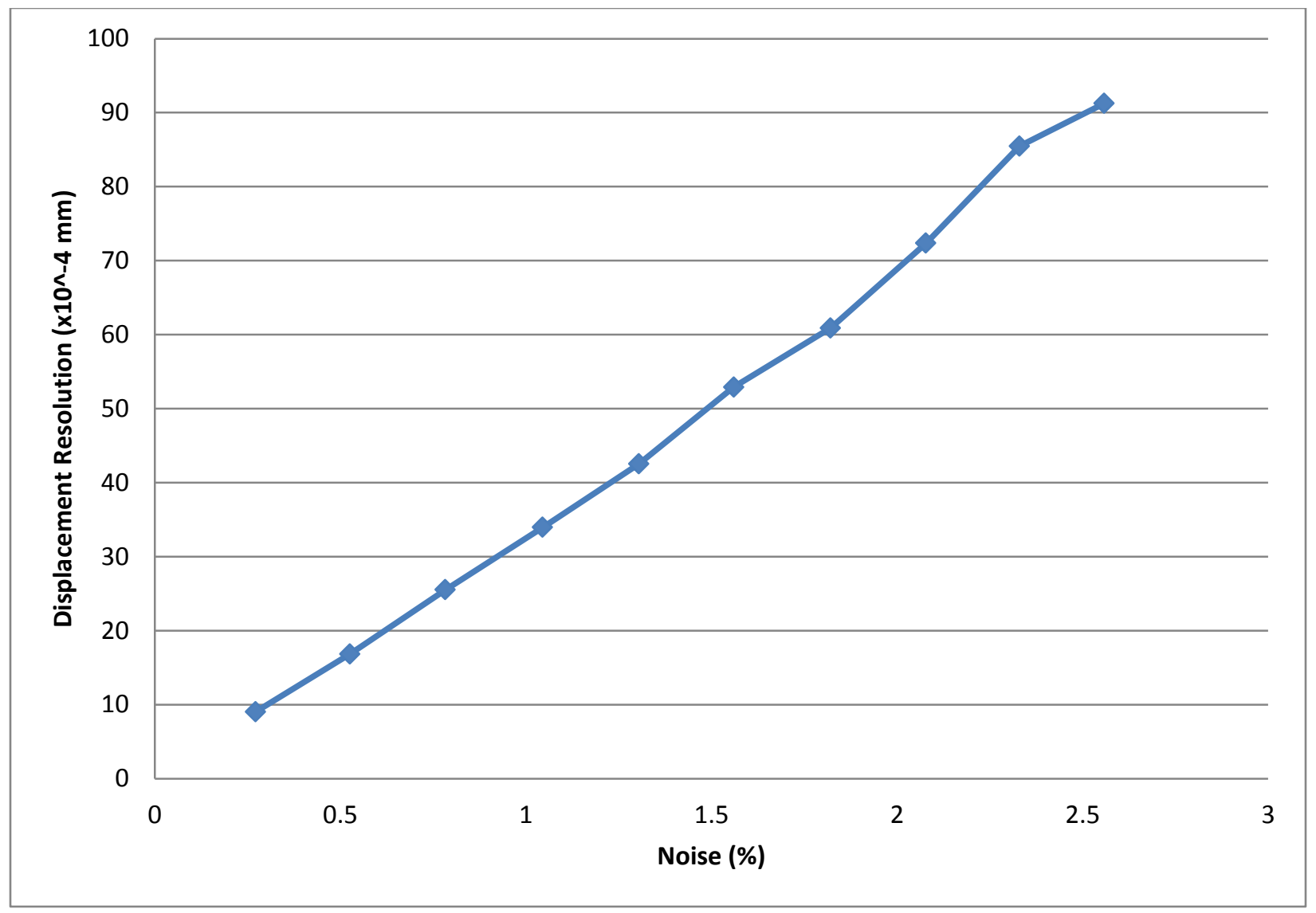

Figure C1: Impact of noise on DIC results

The relationship between noise and displacement is close to linear and thus as camera noise increases so does the resolution of the DIC setup for displacements and strains. It is important to evaluate the noise in each camera before performing the experiment and eliminating high levels of noise by adjusting the lighting. Ideally the noise should be below $1 \%$.

Area of Interest (AOI)- is the area where the user wishes to calculate displacements and strain for the test subject. Data is only calculated up to $1 / 2$ the subset away from the edge of the AOI. 
Subset- shape that encloses a set neighbourhood of pixels for which an independent answer is given at the center of the subset within the AOI. Each independent answer is located a step size apart and therefore a displacement given for a subset is an average displacement among the subset region. The size of the subset determines the smallest displacement feature that can be resolved and is dictated by the speckle pattern.

Step-size- The distance between subset measurements.

For post-data processing the resolution and spatial resolution of displacement and strain must be obtained. Resolution refers to the smallest value of the measurand which can be detected above noise and therefore can be found by recording the standard deviation of displacements and strains for static pre-experiment images. Spatial resolutions refer to the smallest distance between two independent measurement points. For strain measurements there is an added parameter that may be recorded known as the Virtual Strain Gauge or VSG which provides a physical representation to the size of strain gauge needed to represent the results.

Figure C2 provides a summary of the calculated parameters mentioned as well as formulas for VSG and spatial resolution for strain. Spatial resolution for displacement is simply the subset size. One parameter that may be chosen by the user during data processing but has not yet been mentioned is the strain window which refers to the \# of data points to be used for strain calculation. The strain window parameter is shown in Figure C2 and affects the calculated value of VSG and strain spatial resolution. 


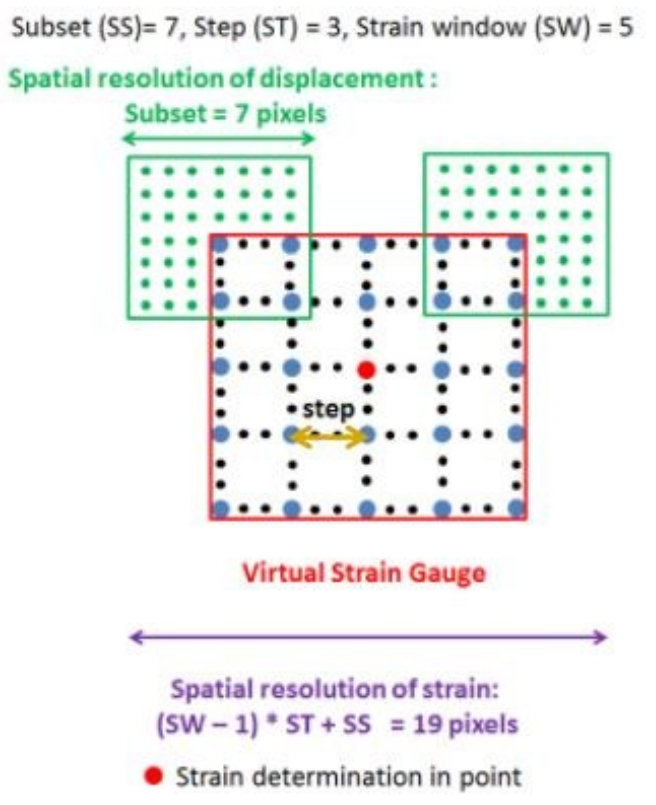

\section{VSG $=[($ Sw-1) $x$ ST $]+1$}

Figure C2: Summary and representation of DIC processing parameters [73]

To convert resolutions and spatial resolutions from pixel values to millimeters there are two options. First, if one knows the field of view (FOV) in millimeters and the resolution of the cameras (ie. 1624 pixels) then a simple division of FOV by the resolution will give the user how many millimeters are in one pixel. Or, the second and more accurate option is to perform a calibration with one of the stereo cameras in the 2D DIC software with a known and visible distance. Thus the 2D DIC software will provide the conversion for the user.

\section{Displacements: Effect of Subset Size on Resolution and Results}

To determine the effect subset size has on DIC displacement results a numerically deformed image according to a 1D-sinusoidal horizontal displacement field is analyzed. 
The period of the sine wave varies from low frequencies ( 210 pixels) to high frequencies (30 pixels) from left to right. The amplitude of the displacement is fixed at 1 pixel.

By varying subset sizes from 11 to 51 in steps of 10, Figures C3 and C4 are created which show how subset size affects the displacement resolutions and amplitude values read at an $\mathrm{X}$-value of 150 pixels and $\mathrm{Y}$ value of 200 pixels. Step size is fixed at 5 . The shape function is also altered between affine and quadratic for both Figures $\mathrm{C} 3$ and $\mathrm{C} 4$.

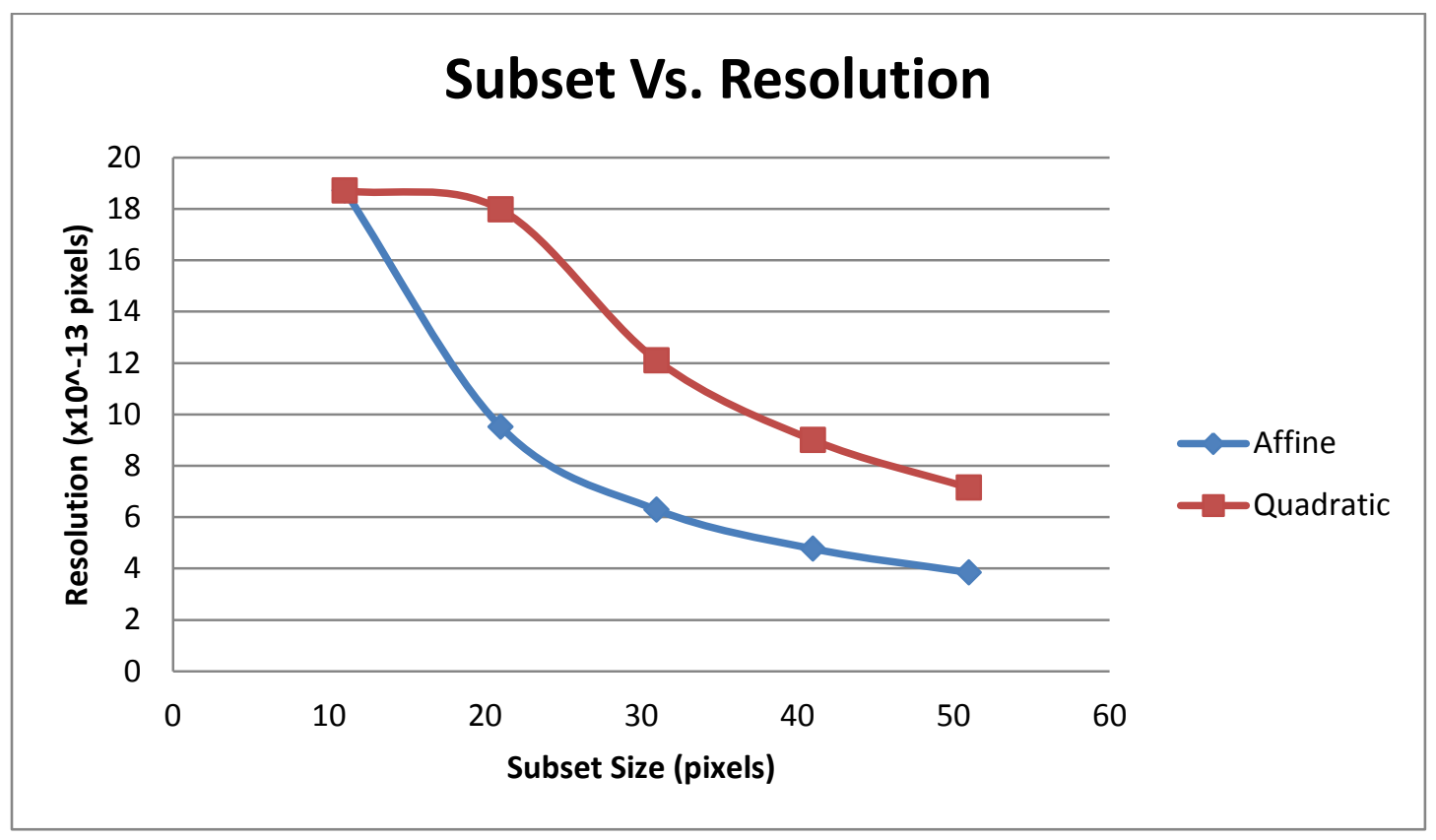

Figure C3: A demonstration of how subset size and shape function affect the resolution 


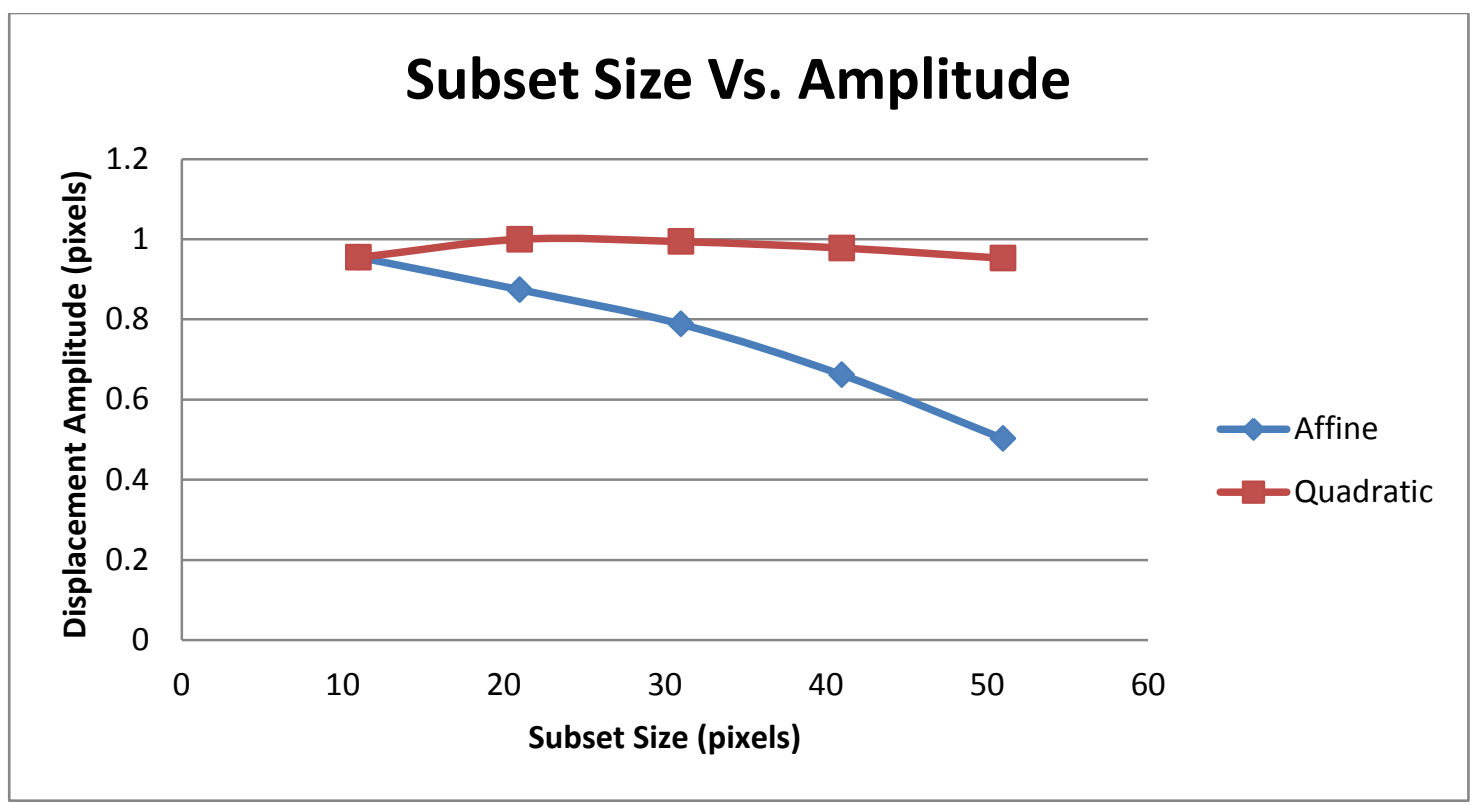

Figure C4: The ability of an increasing subset size and varying shape function to capture displacement gradients

Figure $\mathrm{C} 3$ proves that as subset size increases, displacement resolution decreases and thus there is less uncertainty in the results, however, Figure $\mathrm{C} 4$ contradicts this higher certainty as larger subsets are unable to obtain the 1 pixel amplitude of the sinusoidal displacement when the shape function is affine. Smaller subset sizes are thus better suited for reading periodic deformations or deformations that may contain gradients when the shape function chosen is affine. Larger subsets tend to smooth the data or average out the periodic amplitudes for affine shape functions. This is further shown in figures $\mathrm{C} 5-\mathrm{C} 8$ where deformation images are shown using subsets 11 and 51 along with the corresponding plots of horizontal displacement along the $\mathrm{x}$-axis of the figure. When the shape function chosen is quadratic the amplitudes measured in Figure $\mathrm{C} 4$ are much closer to 1 , however, the resolutions are larger compared to the affine shape function in Figure 
C3. Also, Figure C9 and C10 show that even with a quadratic shape function the higher frequency sinusoidal displacements are not captured very well with a large subset of 51 .

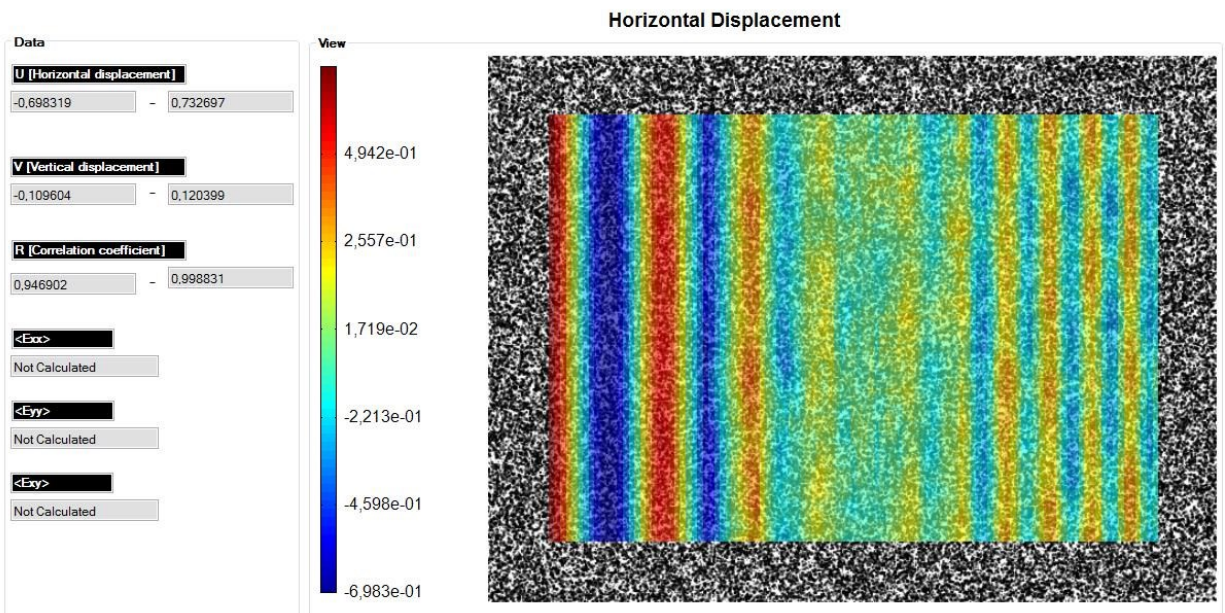

Figure C5: Displacement field using a subset size of 51

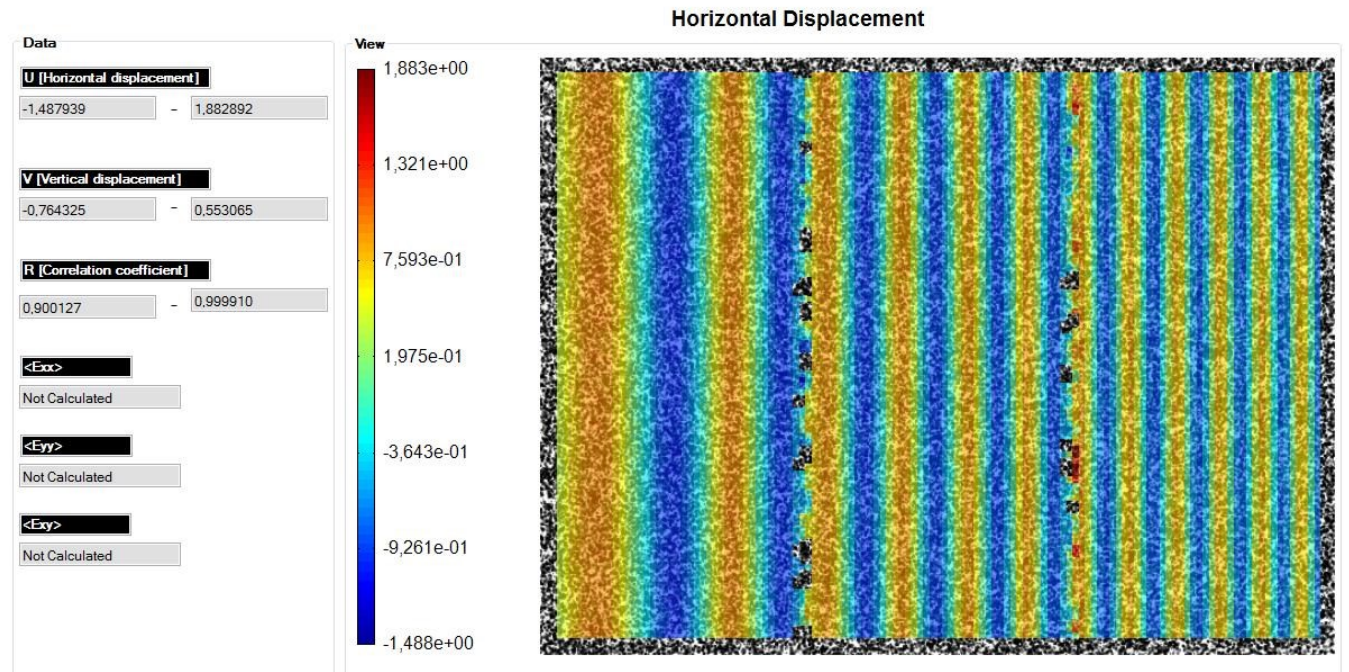

Figure C6: Displacement field using a subset size of 11 


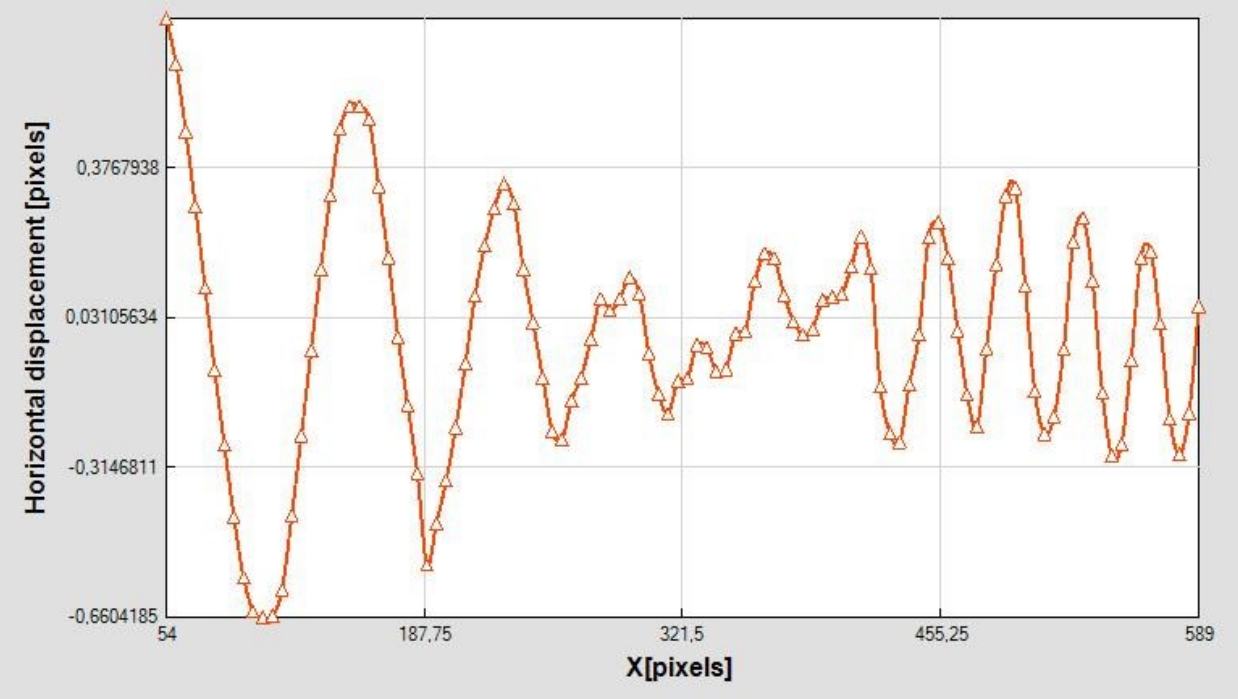

Figure C7: Horizontal displacement pattern retrieved using a subset size of 51 and affine shape function

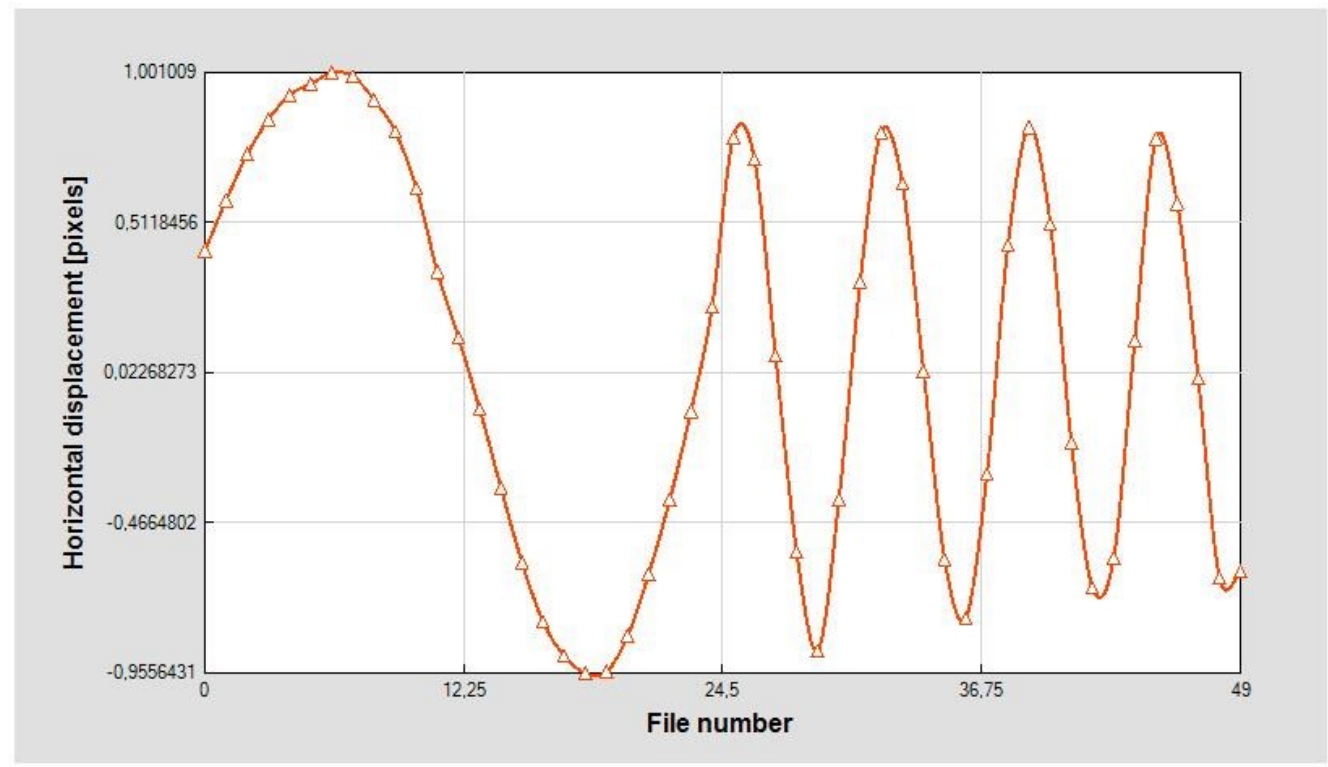

Figure C8: Horizontal displacement pattern retrieved using a subset size of 11 and affine shape function 


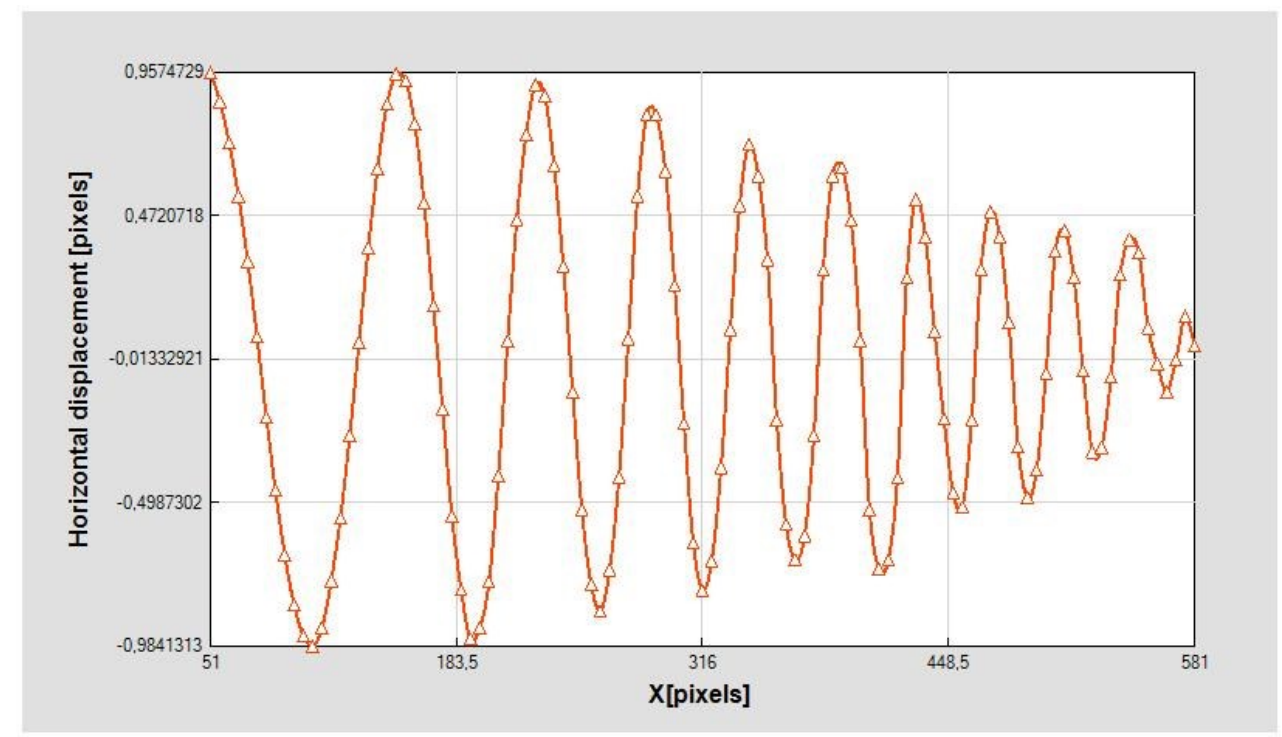

Figure C9: Horizontal displacement pattern retrieved using a subset size of 51 and quadratic shape function

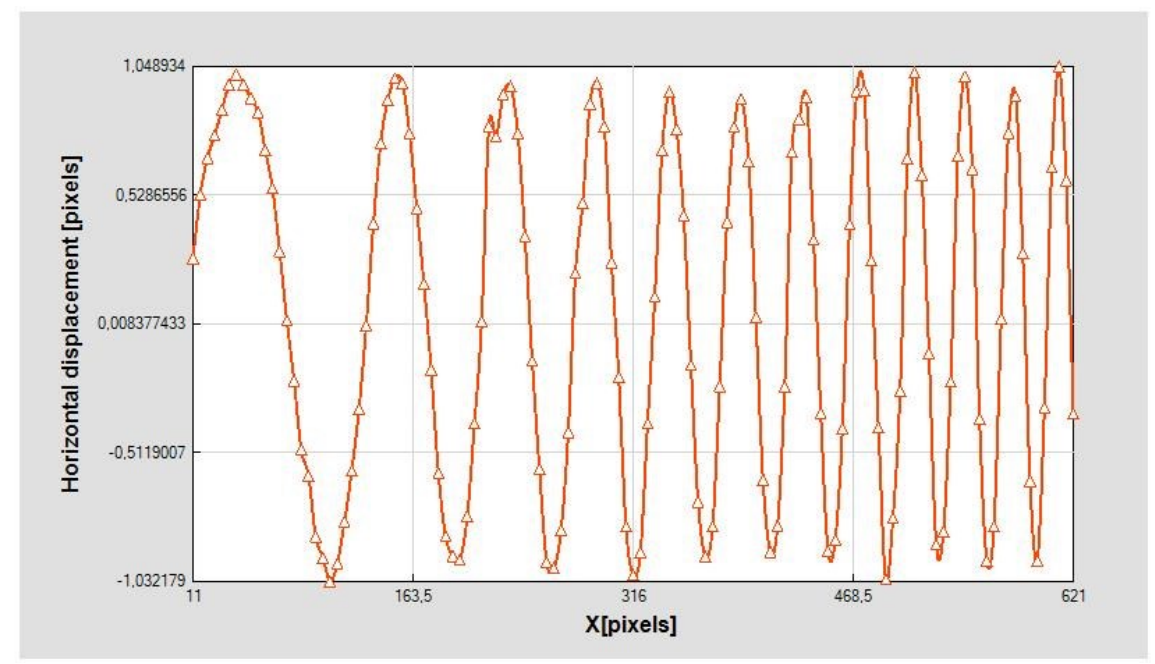

Figure C10: Horizontal displacement pattern retrieved using a subset size of 11 and quadratic shape function

Strains: Effect of user determined parameters on results

Before analyzing DIC results the user must know what effect parameters such as subset size, strain window, and VSG size have on the results. Thus, an analysis has been 
performed on a numerically deformed image with a 1D-sinusoidal horizontal strain field. The period of the sine wave again varies from low frequencies of 250 pixels to high frequencies of 80 pixels from left to right in the image. The amplitude of the strain is fixed at 0.01 .

The impact of several parameters on the strain results will now be analyzed.

1. Impact of strain window

With a subset size of 21 and a step size of 5 , the strain window was varied at 11, 15, and 21 to create Figures $\mathrm{C} 11$ and $\mathrm{C} 12$. Or it may be viewed that the subset was held constant while the VSG size varied. Amplitudes were obtained at an $\mathrm{x}$ value of 180 and y value of 200.

\section{Impact of Subset Size}

The strain window dimensions are fixed at 11 pixels and step size is fixed at 5 , thus the VSG is fixed. Meanwhile the subset is varied between 11 and 51 in steps of 10. Results are plotted in Figures $\mathrm{C} 11$ and $\mathrm{C} 12$.

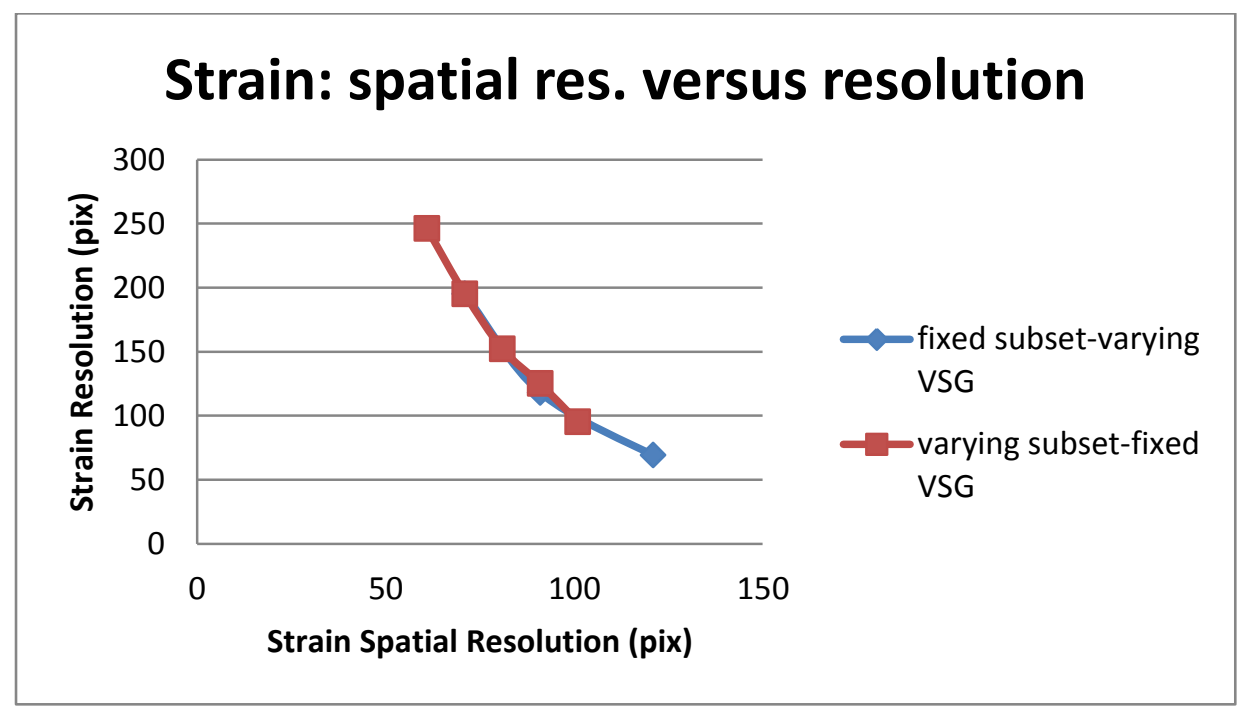

Figure C11: DIC processing parameters effect on strain resolution 


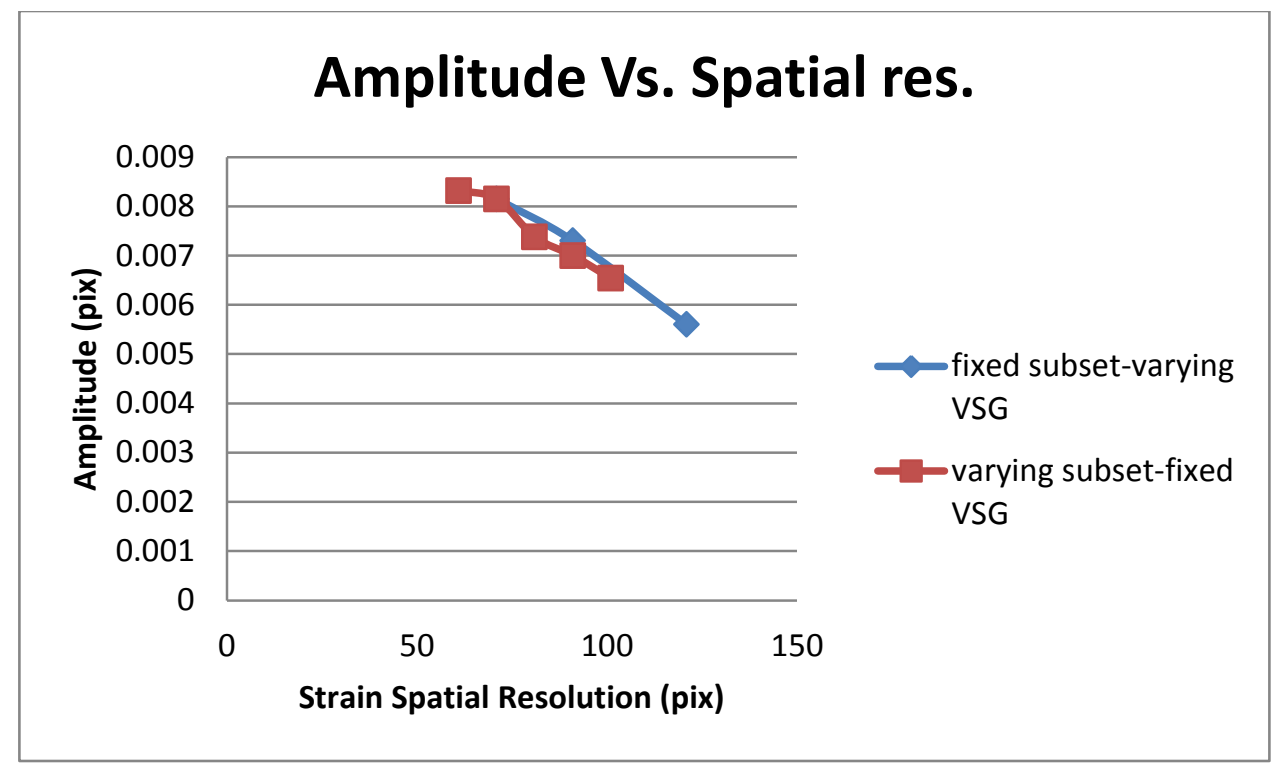

Figure C12: DIC processing parameters effect on high frequency strain amplitude correlation

Figures $\mathrm{C} 11$ and $\mathrm{C} 12$ show that as subset size and strain window size increase so does the spatial resolution which causes the resolution to decrease, but causes the sinusoidal amplitude detected to decrease as well. Therefore, large strain spatial resolutions cause too much smoothing or averaging in the data causing losses of gradient results.

\section{Impact of VSG Dimensions}

If the VSG dimensions are held constant along with subset size so that only strain window and step size may be altered, the corresponding strain resolutions and strain amplitudes determined will be similar as spatial resolution remains almost the same. This is proven in Table 12 where VSG is held at approximately 71 pixels as strain window and step size are varied. Since the spatial resolution remains unchanged and is fairly large, the 0.01 sinusoidal strain amplitude is unobtainable due to smoothing of the results. 
Table C2: Changing VSG dimensions

\begin{tabular}{|c|c|c|c|c|c|c|}
\hline $\begin{array}{c}\text { VSG } \\
\text { (pix.) }\end{array}$ & $\begin{array}{c}\text { Subset } \\
\text { (pix.) }\end{array}$ & $\begin{array}{c}\text { Step } \\
\text { (pix.) }\end{array}$ & SW (pix.) & $\begin{array}{c}\text { Spatial } \\
\text { Res. (pix.) }\end{array}$ & $\begin{array}{c}\text { Strain } \\
\text { Amp. }\end{array}$ & $\begin{array}{c}\text { Strain } \\
\text { Res. }\end{array}$ \\
\hline 71 & 21 & 10 & 8 & 91 & 0.0064 & 91 \\
\hline 70 & 21 & 3 & 24 & 90 & 0.00676 & 117 \\
\hline 73 & 21 & 6 & 13 & 93 & 0.00663 & 111 \\
\hline
\end{tabular}




\section{References}

[1] J. D. Cass. Sheet Hydroforming of Aluminium Body Panels [Online]. Available: http://www.jandngroup.com/wp-content/uploads/2012/01/F-Edited-Cass-Paper.pdf.

[2] R. Wohlecker et al., "Determination of Weight Elasticity of Fuel Economy for ICE, Hybrid and Fuel Cell Vehicles,” SAE Technical Paper 2007-01-0343, Apr. 2007.

[3] Lotus Engineering Inc., "An Assessment of Mass Reduction Opportunities for a 2017 - 2020 Model Year Vehicle Program,” Aug. 2012.

[4] L. Brooke. (2014, Jan. 10). The F-150's Aluminum Diet [Online]. Available: http://www.nytimes.com/2014/01/12/automobiles/autoshow/the-f-150s-aluminumdiet.html?_r=0.

[5] J. Trop. (2014, Sept. 22). Toyota's big leap to aluminum [Online]. Available: http://www.autonews.com/article/20140922/OEM01/309229953/toyotas-big-leap-to$\underline{\text { aluminum }}$

[6] M. de Paula. (2014, May 31). Aluminum Inferior To Steel? Not On The 2015 Ford F150 [Online]. Available:

http://www.forbes.com/sites/matthewdepaula/2014/05/31/aluminum-inferior-to-steel-noton-the-2015-ford-f-150/.

[7 ] P. Rall. (2015, Apr. 7). Ford Breaks Down 2015 F150 Weight Savings [Online]. Available: http://www.torquenews.com/106/ford-breaks-down-2015-f150-weightsavings.

[8] P. Weissler. (2014, Nov. 18). 2015 F-150 aluminum body creates challenge for auto body shops [Online]. Available: http://articles.sae.org/13693/. 
[9] M. Williams. (2014, Nov. 17). Ford's Switch to Aluminum F-150 Increases Costs $\$ 500$ a Truck [Online]. Available: http://news.pickuptrucks.com/2014/11/fords-switch-toaluminum-f-150-increases-costs-500-a-truck.html.

[10] E. Ghassemieh, "Materials in Automotive Application, State of the Art and Prospects," in New Trends and Developments in Automotive Industry, Rijeka, Croatia, InTech, 2011, ch. 20, pp.365-394.

[11] P. Du Bois et al., "Vehicle crashworthiness and occupant protection," The American and Iron Steel Institute, Tech. Rep., Southfield, MI, 2004.

[12] A. Wheatley et al., "Development of Low-Cost Carbon Fibre for Automotive Applications," in Advanced composite materials for automotive applications- Structural integrity and crashworthiness, John Wiley and Sons, West Sussex, UK, John Wiley and Sons, 2014, ch. 3, pp. 51-72.

[13] S.C. Davis et al. "Transportation Energy Data Book: Edition 32," Center for Transportation Analysis, Energy and Transportation Science Division, Oak Ridge National Laboratory, Oak Ridge, TN, Rep. ORNL-6989, July 2013.

[14] C.D. Warren, "High Volume Vehicle Materials," US Low Carbon Vehicles Workshop, Georgia Technological University, Atlanta, Georgia, 2012.

[15] J. Hirsch, "Automotive Trends in Aluminum- The European Perspective," Mater Forum, 28, 15-23, 2004.

[16] ASM Aerospace Specification Metals Inc. Aluminum 6061-T6; 6061-T651 [Online]. Available: http://asm.matweb.com/search/SpecificMaterial.asp?bassnum=MA6061t6. [17] 6016-T4 Aluminum [Online]. Available: http://www.makeitfrom.com/materialproperties/6016-T4-Aluminum/. 
[18] ASM Aerospace Specification Metals Inc. Aluminum 5052-H32 [Online].

Available: http://asm.matweb.com/search/SpecificMaterial.asp?bassnum=MA5052H32.

[19] F. Moller et al., "Novel method for joining CFRP to aluminium," Phys

Proc, 5, 37-45, 2010.

[20] W. Homberg et al., "Combined Forming of Steel Blanks with Local CFRP

Reinforcement," in 10th International Conference on Technology of Plasticity, Aachen, Germany, 2011.

[21] J.G. Broughton et al., "Carbon-fibre-reinforced plastic (CFRP) strengthening of aluminium extrusions," Int J Adhesion Adhesives, 17, 269-278, 1997.

[22] B. Klein, "Leichtbau-Konstruktion," Germany, Vieweg Teubner, 2011.

[23] C. Rans, "The role of rivet installation on the fatigue performance of riveted lap joints," Doctoral Thesis, Mechanical and Aerospace Engineering, Carleton University, Ottawa, Canada, 2007.

[24] J. Laliberte, "Investigation of low-velocity impact damage in fibre metal laminates," Doctoral Thesis, Mechanical and Aerospace Engineering, Carleton University, Ottawa, 2002.

[25] C. Pitzer et al., "Hybrid metal laminate manufacturing planning- evaluation and assessment," Department of Materials Science and Engineering, University of California, Los Angeles, CA, Tech. Rep., 2008.

[26] F. Moriniere, "Low-velocity impact on fibre-metal laminates," Doctoral Thesis, Faculty Mechanical, Maritime and Materials, Delft University of Technology, Delft, NL, 2014. 
[27] A. Vlot et al., Fibre Metal Laminates: An Introduction, Norwell, MA, Kluwer Academic Publishers, 2001.

[28] R.C. Alderliesten, "Fatigue crack propagation and delamination growth in Glare," Doctoral Thesis, Faculty Mechanical, Maritime and Materials, Delft University of Technology, Delft, NL, 2005.

[29] C.C. Chamis et al., "Impact resistance of unidirectional fiber composites," in Composite Materials: Testing and Design Conference, Anaheim, CA, April 1971.

[30] Y. Liu et al., "Effects of constituents and lay-up configuration on drop-weight tests of fiber-metal laminates," Appl Comp Mater, 17, 43-62, 2010.

[31] A. Vlot, "Impact tests on aluminium 2024-T3, Aramid and glass reinforced laminates and thermoplastic composites," Delft University of Technology, Report LR534.

[32] A. Vlot, "Impact loading on fiber-metal laminates," Int J Impact Eng, 18, 291-307, 1996.

[33] M. Sadighi et al., "Experimental and numerical investigation of metal type and thickness effects on the impact resistance of fiber metal laminates," App Comp Mater, 19, $545-559,2012$.

[34] M. Sadighi et al., "Impact resistance of fiber-metal laminates: A review," Int $J$ Impact Eng, 49, 77-90, 2012.

[35] G. Reyes et al., "Manufacturing and mechanical properties of thermoplastic hybrid laminates based on DP500 steel," Comp Part A, 40, 176-183, 2009.

[36] A. Sexton et al., "Stretch forming studies on a fibre metal laminate based on a selfreinforcing polypropylene composite," Comp Struct, 94, 431-437, 2012. 
[37] P. J. Shadbolt et al., "A Preliminary Investigation of Plate Perforation by Projectiles in the Subordnance Range," University of Oxford, UK, Rep. 1372/81, 1981.

[38] F. J. Bradshaw, “Impact Resistance of Carbon Reinforced Plastics,” MOD, Rep. RAE TR 72240, 1972.

[39] J. G. Avery, "Design Manual for Impact Damage Tolerant Aircraft Structures," NATO, Rep. AGARD-AG-238, 1981.

[40] P. W. Manders et al., "A parametric study of composite performance in compression- after-impact testing,” SAMPE J, 22, 47-51, 1986.

[41] A. Rotem, "Residual flecural strength of FRP composite specimens subjected to transverse impact loading," SAMPE J, 24, 19-25, 1988.

[42] S. Agrawal et al., "Impact damage on fibre-reinforced polymer matrix composite - A review," J Comp Mater, 48, 317-332, 2013.

[43] W. J. Cantwell et al., "The Impact Resistance of Composite Materials-A Review," Comp, 22, 347-362, 1991.

[44] S. Abrate, "Impact on Composite Structures," New York, NY, Cambridge University Press, 1998.

[45] G.B. Chai et al., "Low velocity impact response of fibre-metal laminates - A review," Comp Struct, 107, 363-381, 2014.

[46] W. Hufenbach et al., "Charpy impact test on composite structures - An experimental and numerical investigation," Compos Sci Tech, 68, 2391-2400, 2008.

[47] A. C. N. Singleton, "On the mechanical properties, deformation and fracture of a natural fibre/recycled polymer composite," Comp Part B, 34, 519-526, 2003. 
[48] S.L. Kessler, "Instrumented Impact Testing of Plastics and Composite Materials," in Instrumented Impact Testing of Plastics and Composite Materials Symposium ASTM STP 936, Houston, TX, Mar. 1985.

[49] L.B. Greszczuk, "Foreign Object Impact Damage to Composites," in The Symposium on Foreign Object Impact Damage to Composites, Philadelphia, PA, 1973, ASTM STP 568.

[50] M. W. Wardle et al., "Drop Weight Impact Testing of Laminates Reinforced with Kevlar Aramid Fibres, E-glass and Graphite," Comp Tech Rev, 5, 4- 10, 1983.

[51] B. Z. Jang, “Advanced Polymer Composites: Principles and Applications,” Taylor and Francis, 1994.

[52] S. Zhu et al., "Low-velocity impact response of fibre - metal laminates Experimental and finite element analysis," Comp Sci Tech, 72, 1793-1802, 2012.

[53] P. Reu et al., "Measurement of steel plate perforation tests with digital image correlation," in Proceedings of the SEM Annual Conference, New Mexico, USA, June 2009.

[54] J. Hammer et al., "Using Digital Image Correlation (DIC) in Dynamic Punch Tests," Exp Mech, 55, 201-210, 2014.

[55] X. Chen et al., "Full Field Strain Measurement of Punch-stretch Tests Using Digital Image Correlation," SAE Int, 5, 345-351, 2012.

[56] M. O. W. Richardson et al., "Review of low-velocity impact properties of composite materials," Comp Part A, 96, 1123-1131, 1996.

[57] B. D. Agarwal et al., "Analysis and Performance of Fiber Composites," 3rd ed. New Jersey, NY, Wiley and Sons, 2006. 
[58] D. Liu, "Impact-induced delamination - a review of bending stiffness mismatching," J Comp Mater, 22, 674-692, 1988.

[59] R. Olsson, "Closed form prediction of peak load and delamination onset under small mass impact," Comp Struct, 59, 341-349, 2003.

[60] P. O. Sjoblom et al., "On low-velocity impact testing of composite materials," $J$ Comp Mater, 22, 30-52, 1988.

[61] L. S. Sutherland et al., "The use of quasi-static testing to obtain low-velocity impact damage resistance of marine GRP laminates," Comp Part B, 43, 1459-1467, 2012.

[62] Standard Test Method for Measuring the Damage Resistance of a Fiber-Reinforced Polymer-Matrix Composite to a Concentrated Quasi-Static Indentation Force, International American Society for Engineers D6264/D6264M-12, 2012.

[63] P. Chen et al., "Failure mechanisms of laminated composites subjected to static indentation," Comp Struct, 75, 489-495, 2006.

[64] J. Zhou et al., "The influence of strain-rate on the perforation resistance of fiber metal laminates," Comp Struct, 125, 247-255, 2015.

[65] L. Sherman. (2001, Oct.). Impact: Which Test to Use? Which Instrument to Buy? [Online]. Available: http://www.ptonline.com/articles/impact-which-test-to-use-whichinstrument-to-buy.

[66] J.L. Roche et al., "Methodology for Selecting Impact Tests of Composite Materials in Automotive Applications," in Instrumented Impact Testing of Plastics and Composite Materials Symposium ASTM STP 936, Houston, TX, Mar. 1985. 
[67] R.Rodi et al., "Characterization of the crack tip behaviour in fibre metal laminates by means of digital image correlation," in 50th AIAA/ASME/ASCE/AHS/ASC Structures, Structural Dynamics and Materials Conference, Palm Springs, USA, 2009. [68] RTP Imagineering Plastics. (2005). TP 287 H Impact-Modified Nylon 6/6 (PA) Carbon Fiber [Online]. Available: http://web.rtpcompany.com/info/data/0200H/RTP287H.htm.

[69] T. Parnanen et al., “Applicability of AZ31B-H24 magnesium in Fibre Metal Laminates - An experimental impact research," Comp Part A, 43, 1578-1586, 2012. [70] J. Mugica et al., "Impact characterization of thermoformable fibre metal laminates of 2024-T3 aluminium and AZ31B-H24 magnesium based on self-reinforced polypropylene," Comp Part A, 61, 67-75, 2014.

[71] G. Kay, "Failure modeling of titanium-61-4V and 2024-T3 aluminum with the Johnson-Cook Material Model,” US Department of Energy, Rep., 2002.

[72] D. Lesuer, "Experimental investigations of material models for Ti-6Al-4V titanium and 2024-T3 aluminum," Lawrence Livermore National Laboratory, Livermore, CA, Rep. DOT/FAA/AR-00/25, 1999.

[73] MatchID, "Practical considerations in DIC measurements," KU Leuven University, NL, 2013.

[74] J. Laliberte et al., “AERO 4608 Composite Materials Class Notes,” Sept. 2013, unpublished.

[75] E.C. Botelho et al. "A review on the development and properties of continuous fiber/epoxy/aluminum hybrid composites for aircraft structures," Mater Res, 9, 247-256, 2006. 
[76] Military Handbook: Metallic Materials and Elements for Aerospace Vehicle Structures, Department of Defense and Federal Aviation Administration, 1998.

[77] F. Moriniere, "Energy distribution in GLARE and 2024-T3 aluminium during lowvelocity impact," in $28^{\text {th }}$ International Congress of the Aeronautical Sciences Energy, Brisbane, AU, 2012.

[78] M.M. Shokrieh et al., "Investigating the transverse behavior of glass-epoxy composites under intermediate strain rates," Comp Struct, 93, 690-696, 2011. [79] ASM Handbook, 10 ed., ASM International Handbook Committee, 1990. 